\title{
Proposal for Enhancement of the CDF II Detector: An Inner Silicon Layer and \\ A Time of Flight Detector
}

\author{
The CDF II Collaboration
}

Submitted to the Fermilab Director and PAC

September 22, 1998 


\section{Contents}

1 Executive Summary $\quad 4$

1.1 Introduction . . . . . . . . . . . . . . . 4

1.2 Layer $00 \ldots \ldots \ldots \ldots \ldots$

1.3 TOF ............................ 6

1.4 Organization of the Proposal . . . . . . . . . . . 7

2 Technical Description of Layer $00 \quad 9$

2.1 Introduction . . . . . . . . . . . . . . . . . 9

2.2 Motivation . . . . . . . . . . . . . . . . . 9

2.2.1 Impact parameter resolution . . . . . . . . . . . 11

2.2 .2 Beyond Run II . . . . . . . . . . . . . . . . 13

2.3 Design ......................... 14

2.3.1 Constraints . . . . . . . . . . . . . 14

2.3.2 Cooling and Support Structure . . . . . . . . 15

2.3 .3 Silicon .................... 19

2.3.4 Readout ................... . . 21

2.3.5 Material .................. 22

2.4 Construction . . . . . . . . . . . . . . . 23

2.5 Alignment and Position Monitoring . . . . . . . . . . . . 24

3 Technical Description of Time of Flight 26

3.1 Overview ........................ 26

3.2 Scintillator and PMT Assembly . . . . . . . . . . . . 28

3.3 Electronics . . . . . . . . . . . . . . . . . . . 30

3.3.1 PMT base ................... 30

3.3 .2 PMT preamplifier ............... 30

3.3 .3 High Voltage . . . . . . . . . . . . 30

3.3 .4 TDC Design . . . . . . . . . . . . . 32 
3.3 .5 Clock Distribution . . . . . . . . . . . . . 34

3.4 Calibration and Monitoring . . . . . . . . . . 35

3.5 Installation and Integration $\ldots \ldots \ldots \ldots \ldots \ldots$

3.5 .1 Mounting Scheme . . . . . . . . . . . . 36

3.5 .2 Cable Routing . . . . . . . . . . . 36

3.6 Event Reconstruction $\ldots \ldots \ldots \ldots \ldots \ldots \ldots$

3.6.1 Association of tracks with PMT's . . . . . . . . 37

3.6 .2 Determination of Interaction $t_{0}$ 's $\ldots \ldots \ldots \ldots$

3.6.3 Kaon Identification using TOF . . . . . . . . . . 39

3.6 .4 TOF System Occupancy . . . . . . . . . . . . . . 40

3.7 Performance Summary of TOF R\&D . . . . . . . . . 40

4 Physics with Layer 00 50

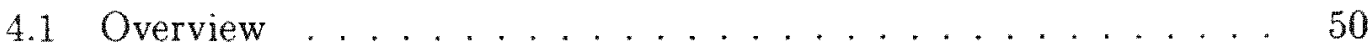

4.2 Physics Studies . . . . . . . . . . . . . . . 51

4.3 Analysis Tools . . . . . . . . . . . . . . 52

4.4 b Tagging in Top Events . . . . . . . . . . . . . . 53

4.4.1 Factors Not Included in the Simulation ... . . . . 53

4.4 .2 Tagging as a Function of $b$ quark $p_{T} \ldots \ldots \ldots \ldots$

4.5 Displaced Tracks in Low $p_{T} b$ Decays . . . . . . . . . . 55

5 Physics with Time of Flight $\quad 57$

5.1 Physics Motivation for TOF . . . . . . . . . . 57

5.1 .1 Overview . . . . . . . . . . . . . 57

5.1 .2 The Unitary Triangle $\ldots \ldots \ldots \ldots . \ldots . \ldots 5$

$5.1 .3 \quad B$ Reconstruction and Flavor Tagging . . . . . . . . 59

5.2 Time-of-Flight Simulation . . . . . . . . . . . . . 62

5.3 Results on $b$ Flavor Tagging using the TOF . . . . . . . 63

5.3.1 Flavor Tagging for $B^{0} / \bar{B}^{0} \rightarrow J / \psi K_{\mathrm{S}}^{0} \ldots \ldots \ldots \ldots 63$

5.3.2 Same-side kaon tagging for $B_{s}^{0} \ldots \ldots \ldots \ldots . \ldots 69$

$5.4 B_{s}$ Mixing Performance with TOF and Layer $00 \ldots \ldots$

5.5 Search for CP asymmetries in the $B_{s}$ system $\ldots \ldots \ldots .77$

5.6 Sensitivity to Gauge-Mediated Supersymmetry . . . . . . . 78

5.7 Other Potential Uses for the TOF System . . . . . . . . . 80

5.8 Summary . . . . . . . . . . . . . . . . . 80 
6 Conclusions $\quad 83$

6.1 Implementation Issues ..................... 83

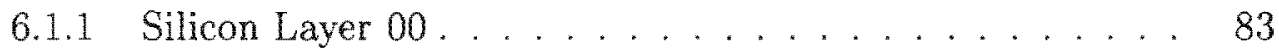

6.1 .2 TOF System . . . . . . . . . . . . . . . 84

6.2 Resources for the Layer 00 and TOF upgrades . . . . . . . . . . . 88

6.3 Summary of Physics Benefits of Layer 00 and TOF upgrades . . 89 


\section{Chapter 1}

\section{Executive Summary}

\subsection{Introduction}

The upgrade of the CDF detector, known as CDF II, is well underway and the collaboration eagerly anticipates its operation with the Tevatron and the Main Injector starting in early 2000. CDF II will be a powerful detector and we expect to carry out a rich program in top, electroweak, exotic, QCD and $B$ physics with the first. $2 \mathrm{fb}^{-1}$ of data collected in Run II. This physics program has been outlined in the Technical Design Report [1] (November, 1996) in which we defined and described the baseline detector upgrade.

The highest priority task of the collaboration is the completion of the 'baseline' upgrade described in the TDR, and a considerable amount of work remains to be carried out in the time remaining. Nevertheless, some of the collaboration's creative energies have been invested in the pursuit of 'beyond the baseline' upgrades. Several potential upgrades have been under consideration by the collaboration. Motivations for these upgrades have included (1) the track record of the Accelerator Division for increasing the available luminosity, (2) studies carried out for TEV33, (3) and of course the initiative of CDF physicists seeking to make the most of the data that can be collected in the first $2 \mathrm{fb}^{-1}$ of Run II and beyond.

Studies of the physics reach of the CDF II detector have continued in parallel with the design and construction of the baseline upgrade. Even as the design of the upgrade is strongly influenced by experience with the Run I CDF detector, we have continued to learn more about the physics potential for CDF II from Run I analyses completed since the TDR. For example, the measurement [2] of the angle $\beta$ in the unitarity triangle using $B^{0} \rightarrow J / \psi K_{S}$ events collected in Run I has increased our confidence in the projections for these measurements in Run II given in the TDR.

In the course of these studies and activities the collaboration has identified two 'beyond the baseline' projects which will significantly enhance the capabilities and scope of the CDF II detector: 
1. The installation of a low-mass radiation-hard single-sided axial-strip silicon detector, called Layer 00 , at a very small radius $(\sim 1.6 \mathrm{~cm}$, i.e. just outside the beam pipe). This detector would employ recent LHC designs for sensors supporting high bias-voltages which enable good signal-tonoise performance even after extreme radiation doses. Each silicon sensor would be $10 \mathrm{~cm}$ long and have a 50 micron strip pitch. To cover the interaction region, 8 sensors are needed in phi and 8 in $\mathrm{z}$ for a total of 64 sensors which corresponds to approximately 16,000 channels or $2 \%$ of the total number of channels in the baseline silicon system (SVXII + ISL). The construction of the detector itself is expected to cost $\$ 208 \mathrm{~K}$ and the required front-end electronics and data acquisition components cost $\$ 233 \mathrm{~K}$ (however, much of this could be covered by SVXII/ISL spares.)

2. The installation of a Time-of-Flight detector (TOF), employing 216 three meter long scintillator bars, with fine mesh photomultiplier tubes on each end, within the space reserved between the outer radius $(138 \mathrm{~cm})$ of the Central Outer Tracker (COT) and the inner radius of the cryostat for the superconducting solenoid magnet. The detector would employ precision ( $25 \mathrm{ps}$ ) timing electronics and achieve a timing resolution for an individual particle of about $100 \mathrm{ps}$. This translates into a $\mathrm{K}-\pi$ separation at the $1 \sigma$ $(2 \sigma)$ level for $p<2.2(1.6) \mathrm{GeV} / \mathrm{c}$. The construction of the detector itself is expected to cost $\$ 687 \mathrm{~K}$ and the electronics, calibration system, cables, etc. would cost $\$ 407 \mathrm{~K}$.

Both projects build upon and extend the capabilities of the CDF II baseline tracking system (SVXII, ISL and COT) and make it possible to more fully take advantage of CDF II's deadtimeless trigger, including a Level 1 track-based trigger (XFT) and a Level 2 impact parameter trigger (SVT). Both projects will significantly expand CDF II's capabilities for physics depending on $b$-tagging, both in terms of the detection of $b$ quarks in jets by virtue of the displaced secondary vertex of the $b$ hadron decay, as well as the determination of the flavor $(b$ or $\bar{b})$ of a neutral $B$ meson at the time of production.

\subsection{Layer 00}

The addition of a low-mass silicon detector layer at small radius (inside the SVX II) will improve the purity of reconstructed tracks and yield more precise and uniform measurements of track impact parameters. The addition of Layer 00 will improve the impact parameter resolution for $1 \mathrm{GeV} / \mathrm{c}$ tracks from $\sim 50$ microns to $\sim 25$ microns, and largely overcome the increased effects of multiple scattering in the SVXII detector compared to SVX' (especially for tracks passing through the high mass regions associated with the SVXII hybrids and electronics). The importance of an inner layer of silicon in this regard was revealed in studies exploring the physics reach of CDFII for Run III [3]. 
Our experience with analyses from Run I have convinced us of the importance of maximizing the acceptance, efficiency, purity and control of systematics for $b$ tagging. The improvement of impact parameter resolution at lower momentum leads to a substantial increase in $b$-tagging efficiency and will also help with the separation of $b$ 's and $c$ 's. Studies show that for $t \bar{t}$ events the efficiency for tagging both $b$ 's improves from $8 \%$ for the Run I SVX' detector to $21 \%$ with SVXII and to $28 \%$ for SVXII augmented with Layer 00 . The improvement in $b$-tagging will lead to corresponding improvements in the study of top physics, including the $t \rightarrow b$ branching ratio and limit on $V_{t b}$, and the search for new physics including the pursuit of an intermediate mass Higgs boson via $H \rightarrow b \bar{b}$.

Our Run I experience has also shown us the importance of vertex resolution for separating exclusive $B$ signals from background. We expect the improved resolution from Layer 00 to provide many benefits from the improved determination of event topologies. Finally the inclusion of Layer 00 improves the vertex-finding resolution contribution to the determination of the proper time for $b$ hadron decays. This is particularly important for measurements of mixing and $\mathrm{CP}$ violation with $B_{s}$ mesons.

Another important motivation for the installation Layer 00 is that it may provide insurance against the loss of the inner most layer of the SVX II detector (Layer 0 ) due to radiation damage. The development of Layer 00 with new radiation hard sensors also allows us to explore this new technology as a potential solution to the problem of vertex tracking in the intense radiation environment of Run III.

\subsection{TOF}

A TOF system would greatly enhance the particle identification capabilities of CDF II. This will increase CDF II's sensitivity to CP violation and $B$ mixing measurements. The increase in sensitivity is especially important for $B_{s}$ mixing, which is a topic that is likely to be unique to the Tevatron during Run II. The TOF system proposed is based on considerable R\&D. It was described at an earlier stage [4] and mentioned as a possible 'beyond the baseline' upgrade in the TDR.

$B$ flavor tagging is a crucial experimental component in the measurement of $C P$ asymmetries in the decays of neutral $B$ mesons to $C P$ eigenstates and in the measurements of $B$ meson flavor oscillations. Information from the Timeof-Flight detector will allow CDF II to improve upon the Same-Side Tagging [5] technique developed and used for measurements of $B^{0}$ mixing and the angle $\beta$ using Run I data $[6,2]$. With TOF we will be able to increase the the flavor tagging efficiency for $B^{0}$ decays by selecting pions over kaons for the Same-Side tag, and also by the inclusion of a tag based on opposite-side kaons. Extending the Same-Side Tagging technique to $B_{s}^{0}$ decays, and selecting kaons over pions for the tag, will more than double the effective tagging efficiency compared to 
techniques available in Run $I$, effectively doubling the available statistics for Run II.

The inclusion of TOF adds an additional important experimental handle: sensitivity to heavy charged particles. The ability to identify pions, kaons and protons with TOF can also be employed to improve the signal to noise in the reconstruction of final states of $B$ hadrons. We have only begun to explore other potential uses.

The measurement of the mixing for $B_{s}^{0}$ mesons serves as an excellent benchmark process, demonstrating the synergy of the combination of the Layer 00 and TOF upgrades. From studies carried out beyond those discussed in the TDR, we have learned that our reach for $B_{s}^{0}$ mixing will be better than previously projected. We intend to measure the $B_{s}^{0}$ mixing frequency using fully reconstructed final states such as $B_{s}^{0} \rightarrow D_{s} \pi$. While it is a challenge to trigger on these final states and isolate the signals from background, they offer the optimal proper time resolution, and the prospect of excellent statistical precision $(<1 \%)$ on the

mixing frequency. For the baseline detector, we expect to be able to observe $B_{s}^{0}$ oscillations up to $x_{s}=45$.

Layer 00 and TOF each individually improve our prospects for observing $B_{s}^{0}$ oscillations, TOF by improving our flavor tagging efficiency, and Layer 00 by improving our ability to resolve the oscillations. Layer 00 also provides some improvements in flavor tagging efficiency. In terms of the effective statistics available for a measurement, the improvements from the two detectors multiply each other, resulting in an extension of our $x_{s}$ reach to 65 .

We look forward to Run II when CDF II will be in the unique position to perform, with one detector, two measurements necessary to make the first test of the closure of the unitarity triangle, namely the measurement of the angle $\beta$ and the measurement of $\left|V_{t d} / V_{t s}\right|$ from a combination of $B^{0}$ and $B_{s}^{0}$ flavor oscillations.

\subsection{Organization of the Proposal}

The proposal is organized as follows: In Chapters 2 and 3 we present the technical aspects of the Layer 00 and TOF upgrade projects. Attention is given to the design and construction challenges as well as the solutions identified for achieving the desired performance.

In Chapters 4 and 5 the motivations for the upgrades and the extended physics reach they afford is then reviewed in more detail with a focus on particular bench mark processes. The importance of Layer 00 and TOF for $B$ physics, particularly $B_{s}$ physics, is presented in Chapter 5 in the context of the projected reach for $B_{s}$ mixing using fully reconstructed hadronic decays.

Chapter 6 provides a discussion of the implementation of these upgrade projects. We also include a tabular breakdown of the costs for the Layer 00 
and TOF upgrades. We briefly review our current understanding in establishing staging strategies and schedules for these projects. We emphasize our efforts to minimize the impact of these projects on the readiness of the baseline detector for operations in early 2000.

We are submitting this proposal in the spirit of the recommendation from the $\mathrm{PAC}$ at the Aspen meeting this summer and the invitation of the Director. The desirability of finding a way of incorporating these two upgrades into CDF II early in Run II, indeed, ideally for the very beginning of Run II, requires serious consideration of the issue of resources, including money, engineers, physicists and time. We are actively pursuing additional collaborators that could provide some of the additional funds and personnel needed to pursue these upgrades as well as contributing to the timely completion of the CDF II baseline detector. With this in mind we seek Stage I approval of the Layer 00 and Time-of-Flight projects at the upcoming PAC meeting in October. 


\section{Chapter 2}

\section{Technical Description of Layer 00}

\subsection{Introduction}

Layer 00 (L00) will be a single-sided layer of low-mass silicon-microstrips at very small radius. The addition of this layer to CDF II will improve track purity and result in more precise and uniform measurements of track impact parameters with corresponding improvements in high $p_{r} b$-tagging, $b$ physics, and overall pattern recognition. The basic readout elements will be individual, single-sided, axial-strip sensors $\sim 10 \mathrm{~cm}$ long. Eight sensors will be mounted end to end along the beam pipe at a radius of $\sim 1.6 \mathrm{~cm}$ as shown in Figure 2.1. The readout electronics will be mounted along the beam pipe at $|z|>40 \mathrm{~cm}$ with fine-pitch kapton cables carrying the signals from the sensors to the readout chips. The sensors will have an implant pitch of $25 \mu \mathrm{m}$ with an alternate strip readout, giving a readout pitch of $50 \mu \mathrm{m}$ and a hit resolution of $\sim 6 \mu \mathrm{m}[7]$. The total number of readout channels will be approximately 16,000 . The sensor design takes advantage of recent work on LHC detectors with high bias voltages enabling good signal-to-noise ratios even after extreme radiation doses.

\subsection{Motivation}

The CDF II tracking system will consist of the Silicon Vertex Detector (SVXII), the Intermediate Silicon Layers (ISL) and the Central Outer Tracker (COT). It will produce major improvements in CDF's ability to tag jets containing $b$ and $c$ hadrons, and reconstruct their decays, as well as its ability to identify the flavor of the $b$ hadrons themselves. We expect higher tracking efficiency and purity than was the case in Run I. The combination of a highly compact SVXII silicon vertex detector and the increased length of all silicon layers, including those of the large radius ISL silicon tracker, will result in a large increase in $b$ daughter track acceptance. Meanwhile, the increase in stereo information available from the COT, ISL and SVXII should greatly enhance 3D reconstruction. The CDF Run II tracking system thus overcomes the main weaknesses of the Run I system. 


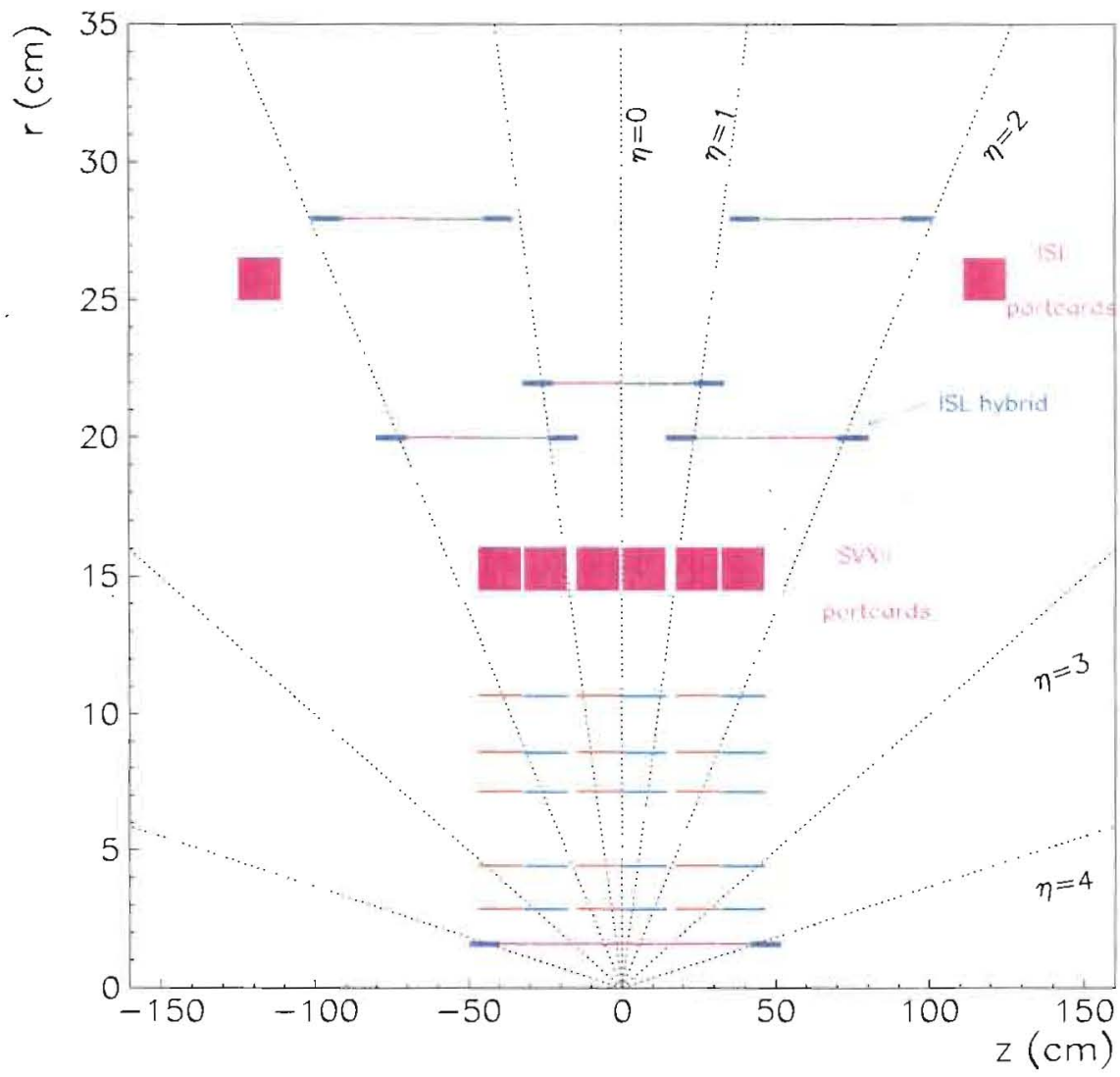

Figure 2.1: Schematic layout of the CDF II silicon tracker with Layer 00 at a radius of $1.6 \mathrm{~cm}$. The radial scale is expanded to display the silicon layers more clearly.

The Run II tracker does however have a few vulnerabilities. Exceptional track acceptance is achieved at the expense of increasing the mass of the active layers. As a result, the impact parameter resolution of the Run II silicon vertex detector for low momentum tracks is reduced in some regions of the detector. A second vulnerability is that it has no clear upgrade path. The innermost layer (L0) of SVXII will be exposed to large amounts of radiation, and although it should survive the next period of data taking $\left(\sim 2 \mathrm{fb}^{-1}\right.$ in Run II) it will need to be replaced for the expected $20 \mathrm{fb}^{-1}$ in Run III. This presents problems of time and resources. For Run III to be useful, it should take place before the LHC detectors begin to produce physics. A significant portion of this window of opportunity will be lost when CDF disassembles to repair SVXII. This should happen only once and for the shortest possible amount of time. Layer 00 would address these issues as described below. 


\subsubsection{Impact parameter resolution}

The low-momentum resolution of CDF's SVX and SVX' detectors was very good with a measured impact parameter resolution of $\sigma^{2}=19^{2}+\left(33 / p_{T}\right)^{2}\left[\mu \mathrm{m}^{2}\right]$. This is to be compared with the expected intrinsic resolution of $\sigma^{2}=13^{2}+$ $\left(34 / p_{T}\right)^{2}\left[\mu \mathrm{m}^{2}\right]$ which is calculated from the detector geometry and material. The difference between the intrinsic asymptotic value $(13 \mu \mathrm{m})$ and the measured value $(19 \mu \mathrm{m})$ is understood as being due to contributions from the primary vertex position and wedge-to-wedge misalignments each of which contributes $\sim 10 \mu \mathrm{m}$. The multiple scattering term $(34 \mu \mathrm{m} \mathrm{GeV} / \mathrm{c})$ is well matched to the measured value.

Similar calculations applied to SVXII show that the intrinsic asymptotic resolution of SVXII is quite good ( $\sim 10 \mu \mathrm{m}$ compared to $13 \mu \mathrm{m}$ for SVX'). However for a large part of the acceptance, SVXII will have worse impact parameter resolution at low momentum than was the case for SVX' due to extra multiple scattering in the readout electronics, which are mounted directly on the surface of the silicon. The readout hybrids add an extra $2.10 \% X_{0}$ of material and cover $33 \%$ of the active area of each SVXII layer. The SVXII bulkheads have even more material, but only cover $6 \%$ of the active area. The impact parameter resolution of a track therefore depends on which region of each SVXII layer it traverses. The amount of material traversed and the corresponding impact parameter resolutions, for normally incident tracks, are listed in Table 2.1 and plotted in Figure 2.2. The table lists the asymptotic resolution, $A$, and the multiple scattering term, $B$. Tracks that are not perpendicular to the beam axis may pass through different regions in different layers, resulting in impact parameter resolutions between the extremes shown.

\begin{tabular}{|c|c||c|c||c|c||c|}
\hline \multicolumn{2}{|c|}{ Region } & \multicolumn{2}{c||}{ SVXII Resolution } & \multicolumn{2}{c|}{ L00 Resolution } & Fraction \\
\hline Region Hit & $\% X_{0} /$ layer & $A_{a}, A_{b}$ & $B_{a}, B_{b}$ & $A_{a}, A_{b}$ & $B_{a}, B_{b}$ & $\%$ \\
\hline Silicon & 0.36 & 9,10 & 34,38 & 6,7 & 22,21 & 61 \\
\hline Hybrid & 2.43 & 9,10 & 66,76 & 6,7 & 27,22 & 33 \\
\hline Bulkhead & 3.43 & 9,10 & 76,88 & 6,7 & 32,28 & 6 \\
\hline
\end{tabular}

Table 2.1: SVXII material, intrinsic impact parameter resolution and acceptance for normally incident particles. Material present is listed as approximate thickness per layer in $\% X_{0}$. The asymptotic $(A)$ and multiple scattering $(B)$ terms are given where $\sigma^{2}=A^{2}+\left(B / p_{T}\right)^{2}\left[\mu \mathrm{m}^{2}\right] . A_{a}$ and $B_{a}$ give the resolution terms for the even numbered wedges while $A_{b}$ and $B_{b}$ give the resolution terms for the odd numbered wedges in which the SVXII silicon is placed at higher radius. The expected resolution terms are given for SVXII alone as well as for SVXII plus L00. The final column gives the fractional coverage for each region of the active area of each SVXII layer. The values for the silicon and hybrid regions are plotted in Figure 2.2.

The expected impact parameter resolution is similarly calculated with L00 


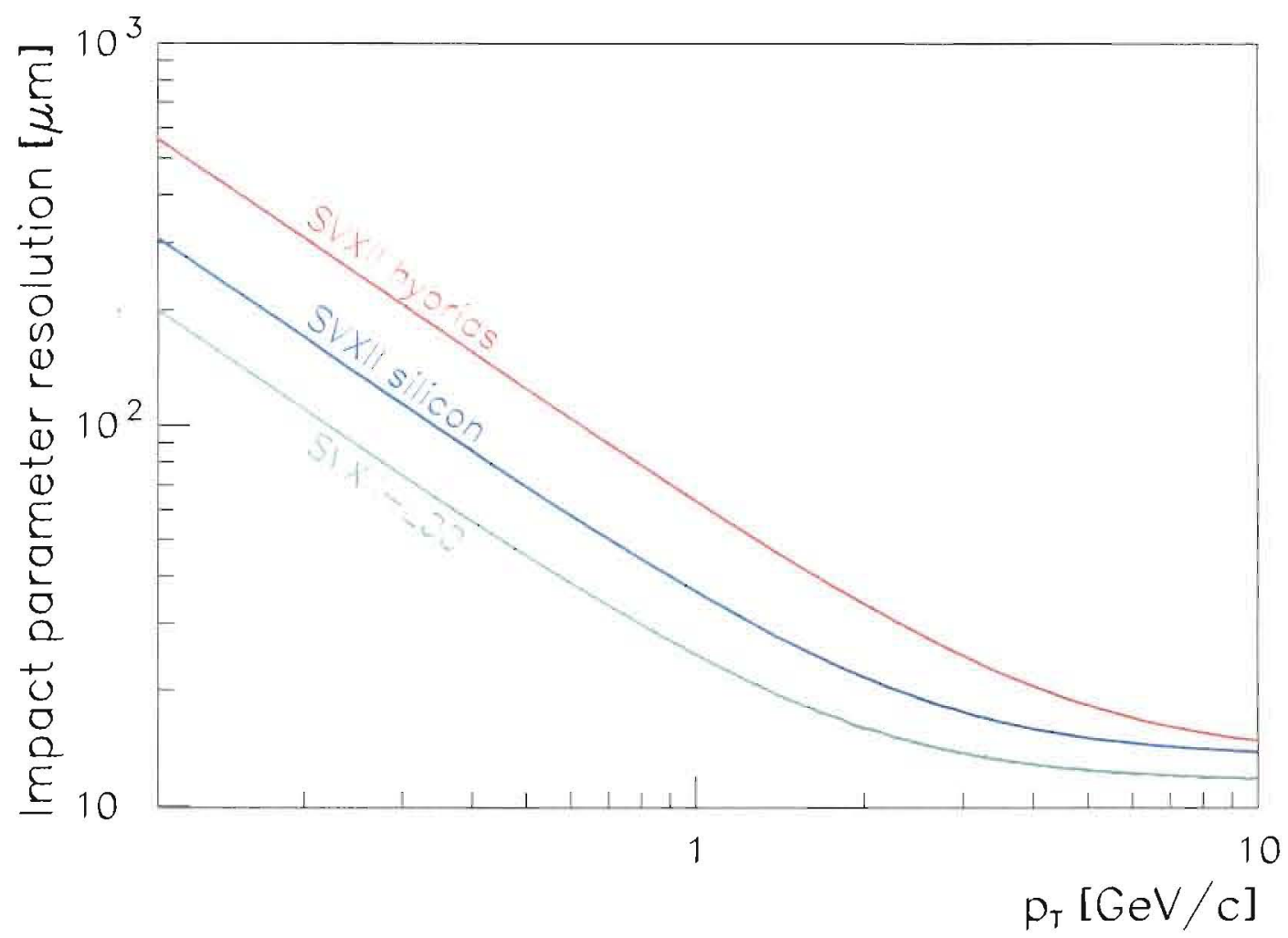

Figure 2.2: Calculated impact parameter resolutions at low momentum for SVXII hybrid regions (top red curve), silicon-only region (middle blue curve). These become the bottom green curve after adding L00. In all cases, $10 \mu \mathrm{m}$ is added in quadrature to the asymptotic term to account for primary vertex uncertainty and wedge-to-wedge misalignments.

included, and the results are also shown in Table 2.1 and Figure 2.2. The improvement in impact parameter resolution provided by L00 is substantial, particularly in the regions of SVXII covered by hybrids as shown in Figure 2.3 and Figure 2.4.

We have also calculated the impact parameter resolution of SVXII for an installed but non-functioning L00 in order to assess the scenario in which L00 does not function in some regions. We find that in the hybrid regions, the multiple scattering constant B is increased by $1 \mu \mathrm{m} \mathrm{GeV} / \mathrm{c}$, and in the silicononly region it increases by less than $2 \mu \mathrm{m} \mathrm{GeV} / \mathrm{c}$. These increases are completely negligible. There is no effect on the asymptotic resolution of SVXII.

Later sections will discuss how the improved impact parameter resolution improves CDF II's $b$ physics capabilities and $b$-tagging efficiency for top studies and Higgs searches. 

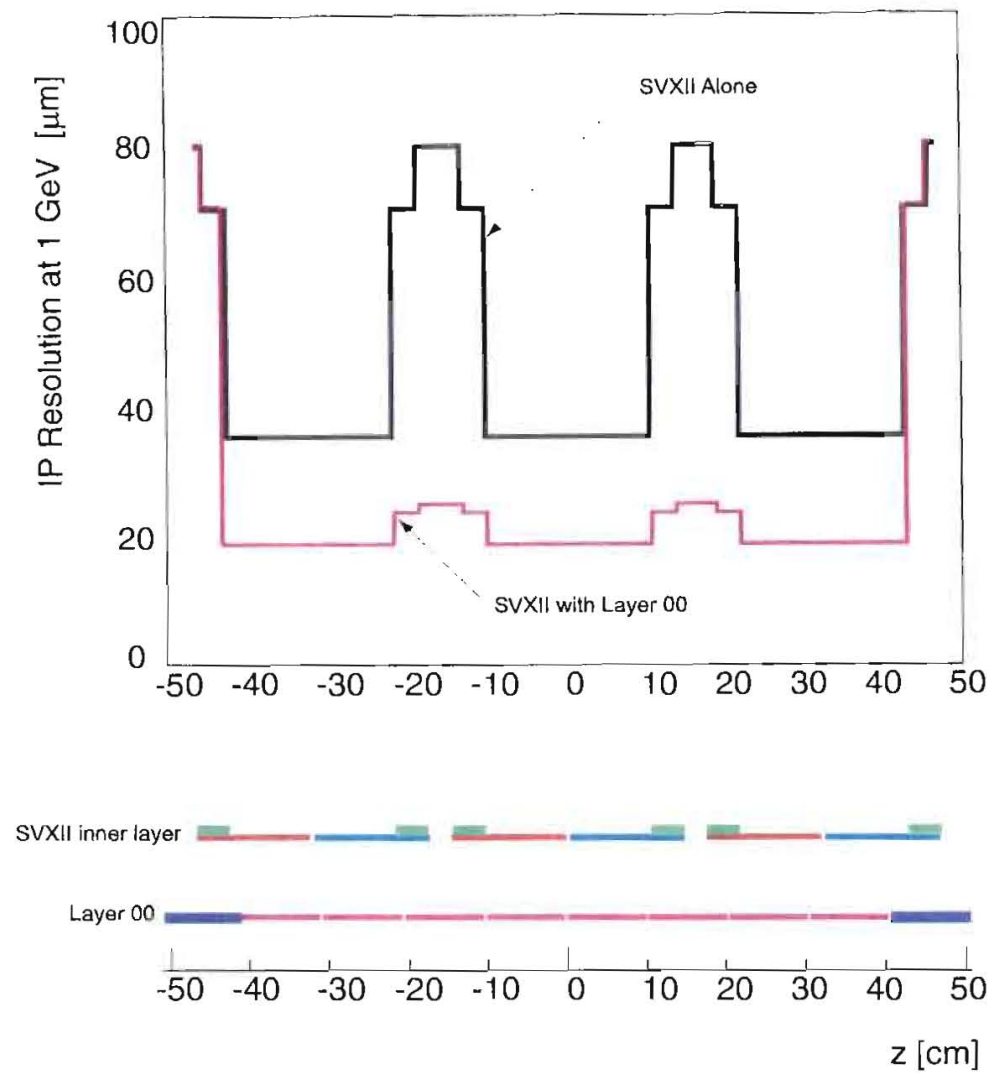

Figure 2.3: Impact parameter resolution for normally incident $1 \mathrm{GeV} / \mathrm{c}$ tracks is shown as a function of z for SVXII and SVXII plus L00 (shown schematically at the bottom). The effect of multiple scattering in the hybrid regions is clearly apparent.

\subsubsection{Beyond Run II}

L00 should outlive the innermost layer of SVXII despite a two-fold greater radiation exposure rate. If the inner layer of SVXII degrades prematurely, for example, L00 would allow the possibility of extended data taking. This would be especially useful if we happen to see signs of a major discovery. In addition, it would provide us experience with new radiation-hard microstrip detectors that could lead to a straightforward upgrade of the inner layers of SVXII for Run III. The current options for inner layer replacements in Run III are pixels, diamonds, and radiation-hard silicon microstrip detectors. While pixel detectors would give potentially outstanding radiation hardness and pattern recognition capability, they also require the most effort, time, and money. It would be a challenge to complete a pixel replacement by the end of Run II. Diamond microstrip detectors, while radiation-hard and continually improving in performance[11], still require some basic R\&D and have not been used in large-scale systems. If viable diamond detectors can be produced in large quantities in the next few years, they will be an extremely attractive option. However, diamond detectors have a smaller pulse height than silicon and would therefore probably require a 


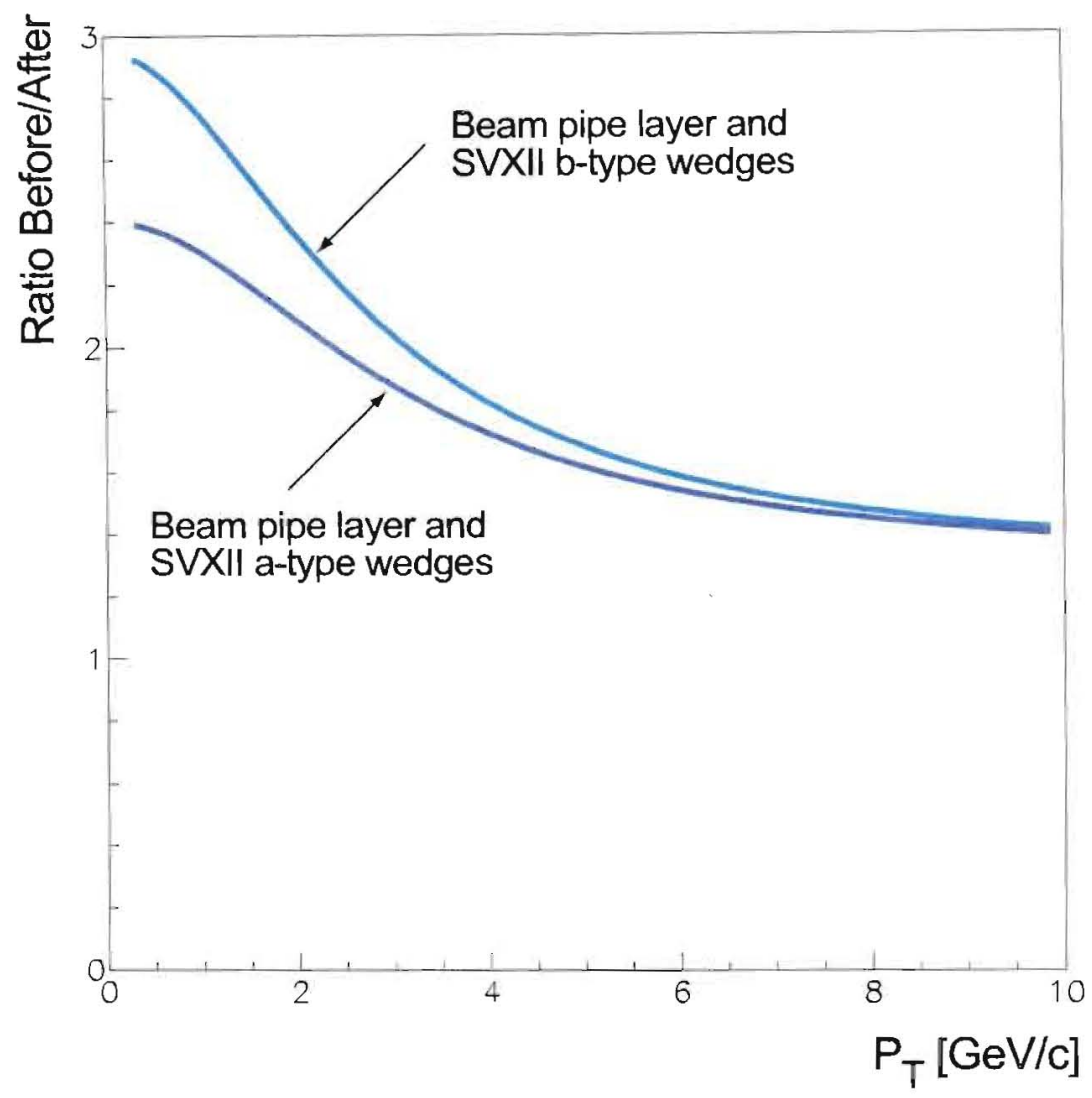

Figure 2.4: Ratio of impact parameter resolution for tracks traversing the SVXII hybrids without and with installation of L00. The bottom curve is for the even numbered wedges where the inner layer of SVXII is at a radius of $\sim 2.5 \mathrm{~cm}$ (a-type wedges). The top curve is for the odd numbered wedges for which the inner layer of SVXII is at a radius of $\sim 3.0 \mathrm{~cm}$ (b-type wedges).

new front-end readout chip.

Silicon microstrip detectors with very high operating voltage and radiation tolerance have been developed for the major LHC experiments[12, 13, 14, 15]. They have been studied and are well understood at this time. Most importantly they could operate with the existing SVX3 front-end chip. Operational experience with these detectors during Run II would give us the knowledge and confidence necessary to use them more extensively in Run III. This would assure us of at least one fast upgrade path.

\subsection{Design}

\subsubsection{Constraints}

L00 must fit between the beam pipe at $r=1.1 \mathrm{~cm}$ and the innermost boundary of SVXII at $r=2.1 \mathrm{~cm}$. This limited radial space imposes many constraints on 
the mechanical design. The radiation at that small radius is intense, the dose rate being double that at the inner layer of SVXII. Silicon exposed to such high doses of radiation must be kept quite cold, typically below $0^{\circ} \mathrm{C}$. This requires a cooling system that directly cools the silicon sensors as well as the electronics. Furthermore, the innermost layer must be very lightweight which constrains not only the cooling system, but also the mechanical support structure which determines the stability and precision of the layer.

An estimate of the radiation dose rate in CDF II as a function of radius can be obtained using the measurement of $0.35 \mathrm{krad} / \mathrm{pb}^{-1}$ obtained in Run I for the innermost layer of SVX together with the observed radial dependence of radiation at $\mathrm{B} 0[9,10]$. This yields

$$
\Phi(r)=2.22 r^{-1.68} \mathrm{Mrad} / \mathrm{fb}^{-1} \text {. }
$$

For a delivered luminosity of $3 \mathrm{fb}^{-1}$ in Run II, this predicts an integrated dose of 1.1 to $1.5 \mathrm{Mrad}$ on the innermost layer of SVXII. Those layers are expected to reach thermal runaway and possibly cease to operate as a result of increased leakage currents at an integrated luminosity of 3 to $4 \mathrm{fb}^{-1}[17]$ and so will have to be replaced for Run III[18].

The radiation situation is obviously worse for $\mathrm{L} 00$. At $1.6 \mathrm{~cm}$, the dose is double that of the innermost silicon in SVXII. We expect $\sim 1 \mathrm{Mrad} / \mathrm{fb}^{-1}$ which implies two constraints. First, extremely radiation-hard silicon must be used, as discussed below. Second, the silicon will itself become a source of heat and must be cooled. Averaging the rise in leakage currents as a function of integrated luminosity measured with the innermost layers of SVX and SVX' in Run I, we obtain[10]

$$
\frac{\delta I}{\delta \int L d t}=21 r^{-1.68} \mu \mathrm{A} / \mathrm{cm}^{2} / \mathrm{fb}^{-1}
$$

at $24^{\circ} \mathrm{C}$. For $r=1.6 \mathrm{~cm}$, this is $9.5 \mu \mathrm{A} / \mathrm{cm}^{2} / \mathrm{fb}^{-1}$. The leakage current decreases with temperature. For instance, at $0^{\circ} \mathrm{C}$ it is $\sim 1.3 \mu \mathrm{A} / \mathrm{cm}^{2} / \mathrm{fb}^{-1}$ and at $-10^{\circ}$ $\mathrm{C}$ it becomes $\sim 0.6 \mu \mathrm{A} / \mathrm{cm}^{2} / \mathrm{fb}^{-1}$. Clearly the silicon must be kept cold to limit the leakage current. Low temperature will also limit reverse annealing[16], which has the potential to ruin detectors in a matter of weeks. This effect can be slowed dramatically by maintaining the silicon at, or below, $0^{\circ} \mathrm{C}$. That is difficult when the readout electronics are mounted on the sensors, but for L00 the electronics would be separated from the sensors, as shown in Figure 2.5. This was the case in the CDF SVX detectors (and will be the case for the CDF II ISL detector[8]). This does not eliminate the need to cool the sensors, but does dramatically reduce the amount of heat that needs to be removed from them, whilst also reducing the material within the tracking volume.

\subsubsection{Cooling and Support Structure}

In order to meet the space, cooling, and material constraints, it is necessary to combine some cooling and support structure functions. We plan to take ad- 


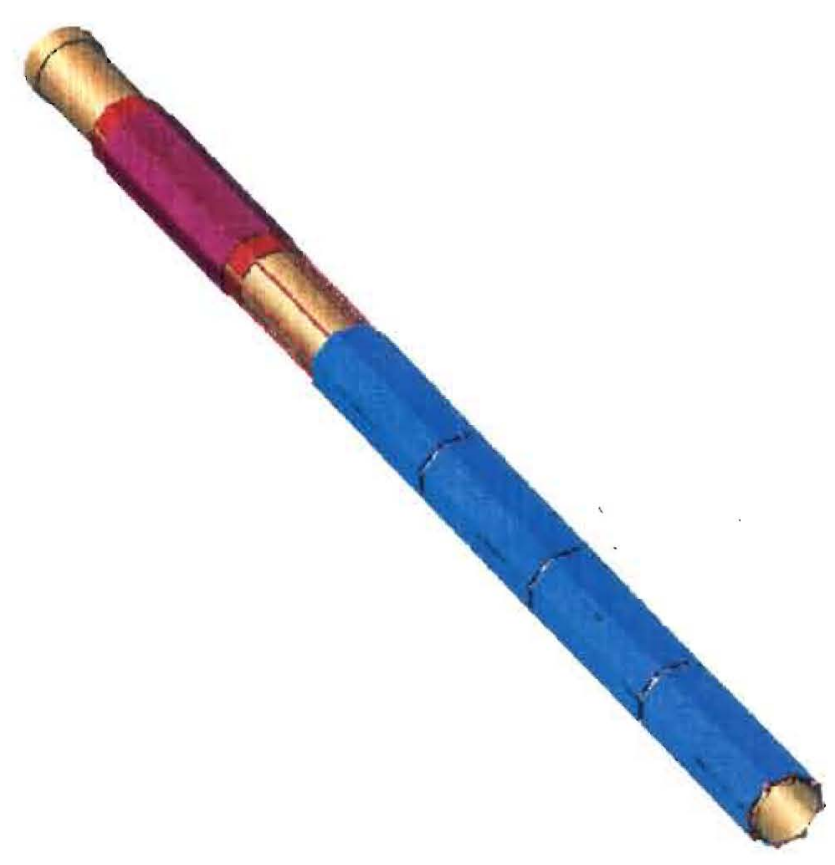

Figure 2.5: Solid model rendering of one half of L00. Shown are 4 sensor groups (blue) starting at $z=0$ and the readout hybrids (magenta) starting at $z \approx 45$ $\mathrm{cm}$. The cooling collar and pipes are shown in red.

vantage of the beam pipe to provide the majority of the support. We plan to wrap the beam pipe in carbon fiber and build our support and cooling layers on top of it. The base layer will have thermally conductive carbon fibers running tangentially around the beam pipe to efficiently carry heat to the cooling channels. Small metal cooling tubes will be interleaved with the carbon fiber support layers as shown in Figure 2.6. The tubes have a $1.5 \mathrm{~mm}$ inside diameter and a wall thickness of $50 \mu \mathrm{m}$ if stainless steel is used or $100 \mu \mathrm{m}$ if aluminum alloy is used. Surrounding the cooling tubes will be thermally conductive carbon fiber to support and cool the silicon layers.

A very thin kapton layer will insulate the back of the sensor from direct contact with the carbon fiber and also carry the bias voltages to the sensor back planes. This design provides excellent structural support and heat transport. However, the carbon fiber layers will need to conform to any irregularities in the surface of the beam pipe. This will be achieved with a precise mandrill and very careful processing.

Coolant will flow from outside the detector to a cooling collar wrapped around the beam pipe which will cool the readout hybrids. The collar will allow a small portion of the fluid to pass through to the cooling channels under the silicon. The flow route will do a U-turn at $\mathrm{z}=0$ as shown in Figure 2.7, then return to the initial distribution collar and back out to the chiller. 


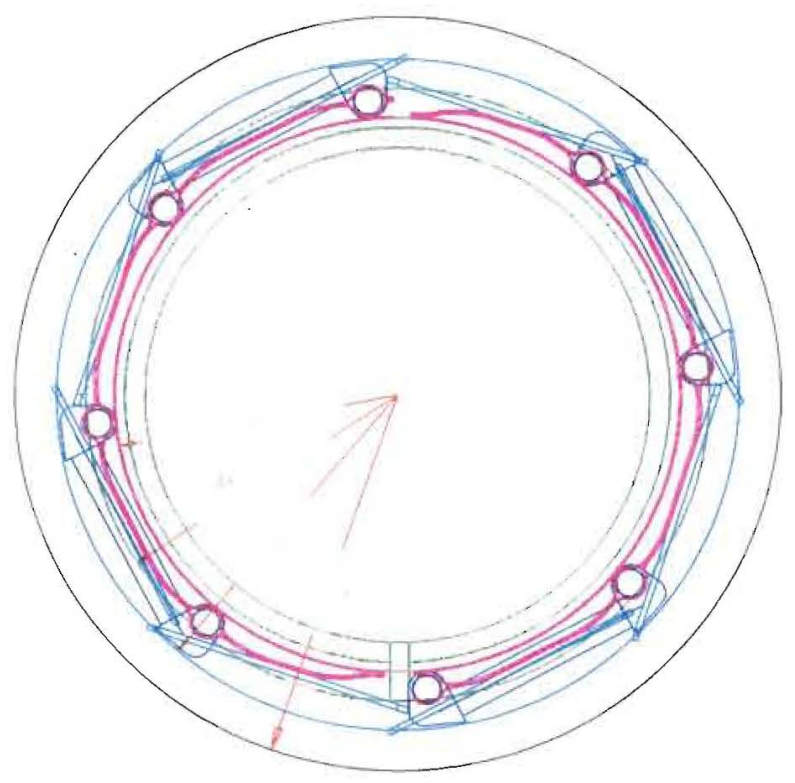

Figure 2.6: Cross sectional view of cooling and support structure with thinwalled metal cooling tubes trapped between plies of thermally conductive carbon fiber.

The SVX3 front-end readout chips consume roughly $0.5 \mathrm{~W}$ each and the layer will contain 64 of them on each end. This is the dominant source of heat but is not a serious concern. The large surface area of contact between the hybrids and the cooling collars, together with the relatively high flow rate of coolant through the collar, will provide ample heat removal.

The largest source of heat for the sensors will be due to heat flow from the SVXII ambient environment $\left(T \approx 15^{\circ} \mathrm{C}\right)$. This will be minimized by a thin aluminized carbon fiber screen between L00 and SVXII which will isolate L00 by preventing convection and reflecting radiation back to SVXII. The screen itself will unavoidably be heated by SVXII and heat up L00 in turn, but the total heat transfer is much reduced. Based on a calculation and mockup we expect this secondary heat load to be $\sim 12 \mathrm{~W}$ in total over all of L00.

Radiation damage is expected to result in an increase in leakage currents of $\sim 1.3 \mu \mathrm{A} / \mathrm{cm}^{2} / \mathrm{fb}^{-1}$ at the radius of L00 for an operating temperature of $0^{\circ} \mathrm{C}$. The heat dissipation per unit area after $10 \mathrm{fb}^{-1}$, assuming an operating voltage for the radiation-damaged sensors of $500 \mathrm{~V}$, is then $6.5 \mathrm{~mW} / \mathrm{cm}^{2}$. The surface area of the entire layer is about $1000 \mathrm{~cm}^{2}$, so the power dissipated by L00 silicon is $6.5 \mathrm{~W}$.

Image currents of the proton and antiproton beams in the Be beam pipe could also be a source of heat. In Run II the Tevatron will have up to 108 bunches of $2.4 \times 10^{11}$ protons and an equal number of bunches with $1.0 \times 10^{11}$ antiprotons. 


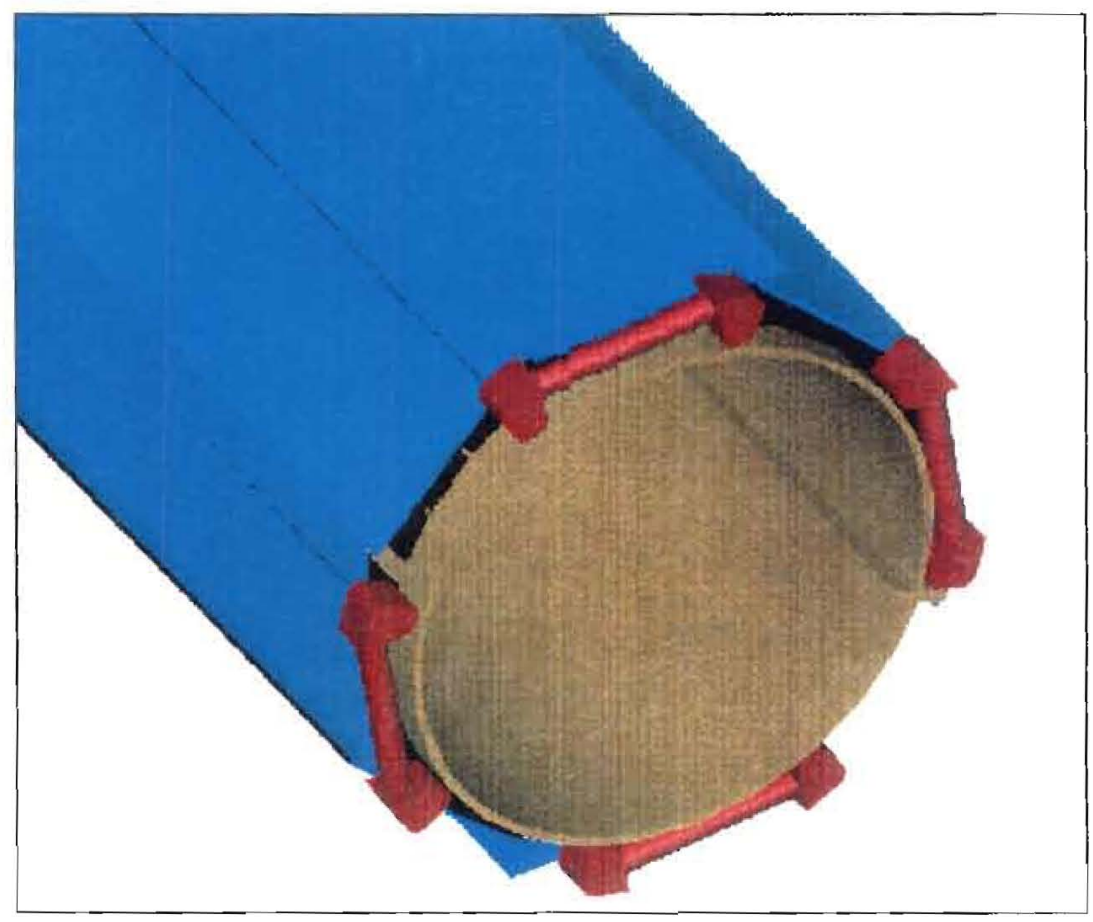

Figure 2.7: Cooling $\mathrm{U}$-turns at $\mathrm{z}=0$ using small cornering blocks and small sections of thin-walled tubing.

The bunch spacing is $132 \mathrm{~ns}$ and the bunch length is roughly $0.2 \mathrm{~m}$. A calculation determines that the power dissipated per unit length of beam pipe by image currents is $0.2 \mathrm{~W} / \mathrm{m}$. Following more careful calculation procedures used by the Fermilab Accelerator division[20] we obtain the range 0.15 to $0.33 \mathrm{~W} / \mathrm{m}$.

As an aside, note that the beam currents could also be a source of noise. Although beryllium is not a very good conductor, for the frequencies associated with the beam currents, the skin depth is $32 \mu \mathrm{m}$. The beam pipe itself has wall thickness of $508 \mu \mathrm{m}$. This should be adequate to shield L00. A more serious concern is that the beam pipe outer surface could act as an antenna. We would protect against this by placing a very thin, isolated layer of aluminum under L00 that would be connected to a quiet ground. This would add as little as $0.06 \% X_{0}$ of material.

Ionizing particles also generate heat in the beam pipe. Based on the expected flux of $2.2 \times r^{-1.68} \mathrm{Mrad} / \mathrm{fb}^{-1}$ and an instantaneous luminosity of $2 \times 10^{32} \mathrm{~cm}^{-2} \mathrm{~s}^{-1}$, we estimate an average of $0.2 \mathrm{~mW} / \mathrm{m}$ of heat from radiation. This rises to $\sim 6 \mathrm{~mW} / \mathrm{m}$ for radiation at the level required to abort the beam $(2 \mathrm{rad} / \mathrm{sec}$ at $r=3 \mathrm{~cm})$.

¿From the above discussions, we estimate the maximum heat transfer to L00 at $Q=(12+6.5+0.5) \mathrm{W}=19 \mathrm{~W}$. With 8 cooling circuits, each circuit would need to be able to remove $2.4 \mathrm{~W}$ of heat after a radiation dose of $10 \mathrm{Mrad}$.

We have calculated the heat removal ability of the L00 cooling system design 
and found no difficulty. To be compatible with the SVXII chillers, the coolant will be a mixture of water and ethylene glycol ( $30 \%$ by weight) at an inlet temperature of $-10^{\circ} \mathrm{C}$. Using $1.5 \mathrm{~mm}$ diameter cooling tubes and assuming twice the expected heat load, the pressure drop is calculated to be 5.5 psi and the temperature drop, inlet to outlet, is $2^{\circ} \mathrm{C}$. To check the results of the calculation, a simple test was run and found to be in reasonable agreement. Calculations of the temperature gradient from coolant to silicon surface are ongoing, but indicate that it should be possible to maintain the silicon at $\sim 0^{\circ} \mathrm{C}$ for a coolant temperature of $-10^{\circ} \mathrm{C}$.

\subsubsection{Silicon}

We intend to make use of recent $R \& D$ performed for the $L H C$ in the development of silicon sensors with high breakdown-voltage. Our sensors will be essentially equivalent to sensors designed for the CMS experiment.[12] These are expected to withstand an ionizing dose at the innermost CMS barrel layer of $\sim 10$ Mrad. Sensors have now been consistently produced by a number of vendors that withstand test beam fluences of this level without more than $\sim 10 \%$ degradation in signal-to-noise ratio $(\mathrm{S} / \mathrm{N})$. In this section we will give a brief technical description of these sensors.

Radiation damage can be classified as either surface or bulk damage in silicon. Surface damage is mostly manifested as holes trapped in either the oxide or oxide-silicon interface. The presence of these holes modifies the oxide field and leads to new energy levels in the forbidden energy gap. The deleterious effects are a decreased inter-strip isolation leading to unwanted sharing of charge between neighboring strips, and increased inter-strip capacitance leading to increased noise. These effects can be countered by applying a bias voltage in excess of that needed for full depletion. The high applied voltage forces free electrons, which are attracted by the oxide space-charge, into the gap between strips. Bulk damage is due to the creation of lattice defects by radiation. These defects can act as electron-hole generation or recombination sites. In general, bulk damage leads to an increase in leakage current, which is linearly related to the integrated particle flux.

Initially the sensor bulk is n-type as a result of a phosphorous (P) dopant which creates donor sites. With irradiation the space charge, which was initially dominated by the P-dopant, becomes increasingly negative. The absolute difference in $\mathrm{P}$-dopant donor site density and radiation-induced acceptor site density is defined as the effective dopant concentration $N_{\text {eff }}$. With continued irradiation, the silicon becomes progressively less $n$-type and eventually inverts to become progressively more $\mathrm{p}$-type. The radiation flux leading to inversion scales with the initial resistivity of the silicon, determined by the initial $\mathrm{P}$-dopant density. At inversion the acceptor and donor concentrations are balanced $\left(N_{\text {eff }}=0\right)$. After inversion the acceptor sites dominate and $N_{\text {eff }}$ can increase indefinitely. The evolution of the effective dopant-concentration is important because it determines 


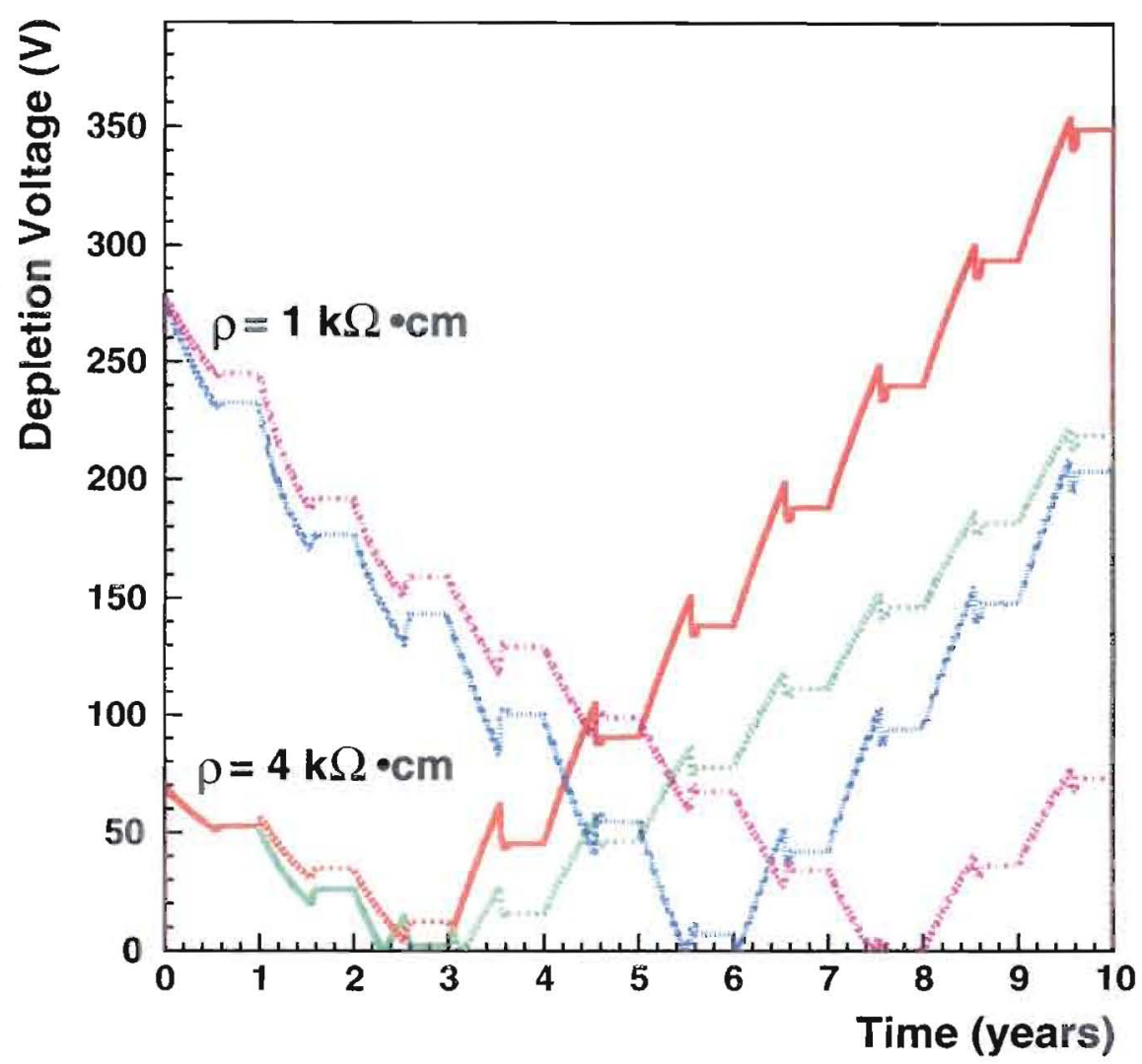

Figure 2.8: The expected evolution of the depletion voltage of CMS sensors with two values of initial resistivity and for radiation doses totaling $1.6 \times 10^{14} \mathrm{n} / \mathrm{cm}^{2}$ and $2.4 \times 10^{14} \mathrm{n} / \mathrm{cm}^{2}$ after 10 years. The radiation doses for L00 are comparable to those for the (much larger radius) CMS microstrip detectors, and although the plot is based on expected running conditions at the LHC, it provides a useful guide to the expected time evolution of the L00 depletion voltages.

the depletion voltage of the device, $V_{\text {dep }}=N_{\text {eff }}\left(e d^{2} / 2 \epsilon\right)$, where d is the thickness of the sensor, $\epsilon$ is its dielectric constant, and $\mathrm{e}$ is the quantum of electric charge. It follows that the depletion voltage of the sensor should initially decrease with radiation to approximately $0 \mathrm{~V}$ at inversion and then increase continually. For large radiation doses such as those anticipated at LHC or by CDF II at small radius, the depletion voltages of sensors is expected to rise dramatically. CMS has developed sensors with bias breakdown-voltages in excess of $500 \mathrm{~V}$. The evolution of the depletion voltage for these sensors operated at the LHC over a period of 10 years with regular intervals of no beam is shown in Figure 2.8.

The LHC research program has resulted in design and processing specifications for silicon sensors to allow the consistent production of high operating voltage sensors. The most significant considerations are the treatment of the edges of the sensors, the implant shapes, and the oxides for both the surface passivation and the coupling capacitors. The edge of the sensor is generally fraught with defects as a result of the cutting process and can easily result in 


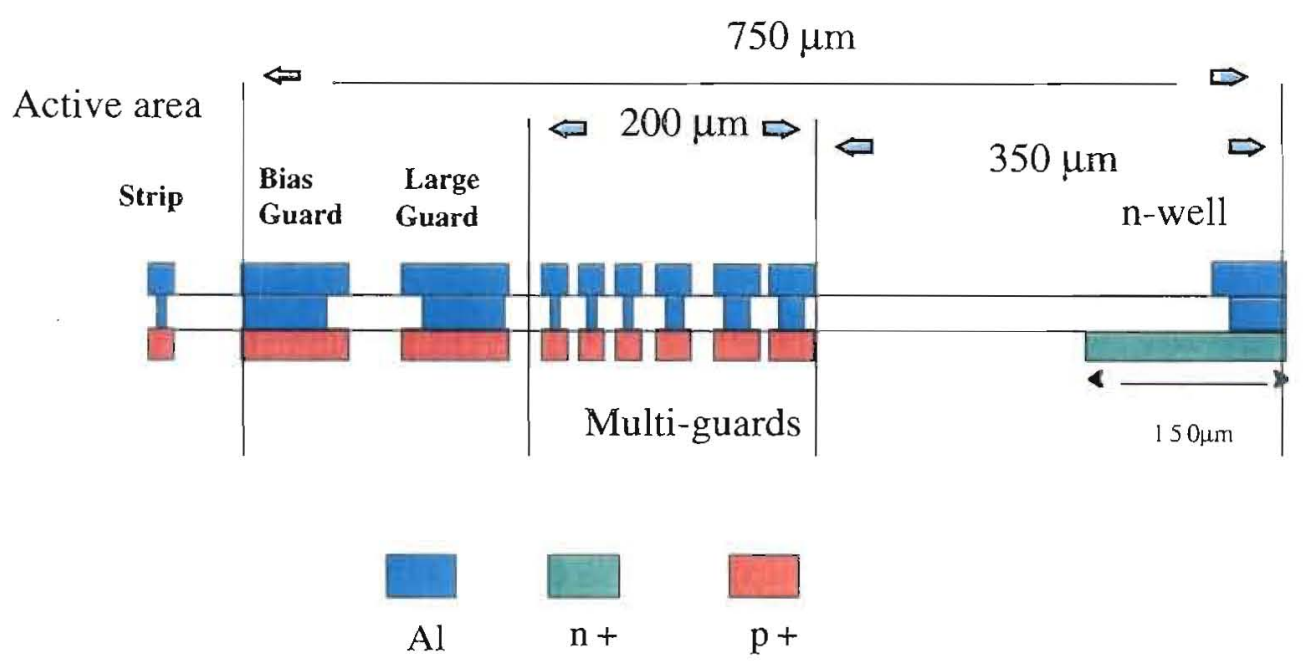

Figure 2.9: Schematic diagram of the CMS sensor guard ring structure.

breakdown across the bulk or act as a significant source of current. To counter these possibilities an $n+$ guard ring is placed on the top surface near the edge. Extending from $350 \mu \mathrm{m}$ to $550 \mu \mathrm{m}$ from the edge is a multiple $\mathrm{p}+$ guard structure. This assures that the electric fields on the surface do not have a large gradient. The overall surface structure is shown in Figure 2.9. The leakage current per strip is required to be less than $0.5 \mu \mathrm{A}$ at 500 volts of bias after irradiation and less than $50 \mathrm{nA}$ before irradiation.

CMS has prototyped sensors with these characteristics from several vendors and has included prototype L00 detectors on masks which will allow us to test the L00 sensor designs in the fall of 1998.

\subsubsection{Readout}

The ability to place the readout hybrids outside the central tracking region is critically important for cooling and space, and also improves the performance by reducing the material within the tracking region. We plan to use lightweight, fine pitch cables to connect the sensors to the readout hybrids. These cables will be kapton with a total thickness of $50 \mu \mathrm{m}$. Traces can be made roughly $20 \mu \mathrm{m}$ wide with up to $50 \mu \mathrm{m}$ pitch. Currently these cables are made at CERN[19] and elsewhere in Europe and the U.S. The traces are $5 \mu \mathrm{m}$ thick copper, with 200 Angstroms of Nickel. Each cable represents only $0.023 \%$ of a radiation length.

With $50 \mu \mathrm{m}$ pitch, the cables have a capacitance of $0.46 \mathrm{pF} / \mathrm{cm}$, to be compared with $\sim 1.0$ (1.4) pF/cm for the silicon detectors with 50 (25) $\mu \mathrm{m}$ pitch. Since the cable lengths for the sensors nearest $z=0$ are quite long, the added capacitance becomes significant in increasing noise. To overcome this, we plan to use two $100 \mu \mathrm{m}$ pitch cables for the longest paths. The capacitance per unit length is then halved and the maximum added capacitance is roughly $8 \mathrm{pF}$ re- 
sulting in a total capacitance of less than $\sim 22 \mathrm{pF}$. This will result in an excellent $\mathrm{S} / \mathrm{N}$ of $\sim 16: 1$ with 396 ns readout time (based on recent SVX3d chip tests).

The readout hybrids are expected to be fairly straightforward adaptations of the ISL hybrids which are in turn based on the design used for SVX and SVX'. They will be manufactured on Alumina which can be thin since the hybrid is single-sided and will lie directly on top of a cooling collar. The hybrids are restricted to be somewhat narrow $(\sim 1.25 \mathrm{~cm})$ but can be fairly long. One possibility is to have each hybrid service a single chip. Eight of these hybrids would be stationed, end to end, at the end of the row of 4 sensors. In the hybrid region, the total material contributed by the electronics is $2.1 \% X_{0}$ in addition to an estimated $0.5 \% X_{0}$ for the cooling collars.

The Data Acquisition (DAQ) for L00 will be identical to the SVXII DAQ[8] for all components downstream of the hybrids. However, the readout configuration will differ from SVXII in order to minimize the DAQ cost while keeping the readout time less than the time for the SVXII readout which will occur in parallel. The LOO sensors are single-sided and shorter than the sensors in SVXII, so they should have, on average, about half as many hits to read out. This allows two sensors to be multiplexed. The multiplexing will be done in $z$ i.e., the data from two sensors at the same $\phi$ but neighboring in $z$ are time multiplexed into a single readout path.

L00 will use the same portcard design as SVXII and ISL. It will require an additional 8 portcards beyond the 102 needed by SVXII and ISL. Four L00 portcards will be mounted on the ISL portcard ring at each end of the detector. L00 can also use the same power supplies as SVXII with one modification. The SVXII power supplies are specified to have a maximum bias voltage of $250 \mathrm{~V}$, while L00 may require a bias voltage above this level by the end of Run II due to radiation damage. The L00 supplies may need to be modified to accommodate this higher voltage. Fortunately, the CAEN supplies are designed with daughter cards providing each of the different voltages needed. This modification will therefore involve only a single daughter card.

\subsubsection{Material}

The total material added by $L 00$ is $\sim 0.6 \%$ of a radiation length for the $85 \%$ of the area without cooling tubes. For the $15 \%$ of the area containing cooling tubes it is $\sim 1 \%$ of a radiation length. These material estimates include $0.32 \%$ from the silicon and $0.07 \%$ from the cables. The material in the hybrid region, which is outside the tracking volume, is $\sim 2.6 \%$ of a radiation length. 


\subsection{Construction}

We intend to map the beam pipe on a precision coordinate measuring machine (CMM) and make a cast to form a dummy pipe with similar sag properties. L00 will be built in two half cylinder sections ("clam shells"), the construction of which will then take place on this dummy pipe to support them in the same way it will be supported in the final installed state.

We will form cylindrical cooling tubes into channels with a press. All parts will then be glued in place using a CMM for precision. Modules will be constructed on a flat, stiff plate with a thin kapton layer. Four sensors and one hybrid group will be glued to the kapton using a simple micrometer adjustment fixture for precision strip alignments. The kapton will also carry conductors to set the back plane voltage of the sensors. After the silicon is placed and glued to the kapton, which is itself attached via tabs to the stiff base plate, the kapton cables can be glued to the detectors and hybrids one by one. Each cable will be wirebonded at both ends before the next cable is installed.

Once the modules are complete, they will be installed on the support structure. A fixture will be used to hold aluminum end pieces, which are pinned to the base plate during module construction. For installation, the end pieces are retained to hold the reference of the strips, but the base plate is removed. A stepper motor and encoder will be used to clock the modules into the proper $\phi$ location. Each module can then be glued to the support structure, and later the end pieces can be removed by cutting the kapton to which they are glued.

With equipment available at the Fermilab Silicon Detector Center (SiDet), and based on past construction experience, we estimate that the detectors can be aligned within modules, and from module to module in the support structure, to $25 \mu \mathrm{m}$. Using available large CMMs, it is possible to map sensor locations relative to one another in 3 dimensions to better than $10 \mu \mathrm{m}$, although such precise measurements are probably not necessary at this stage as final alignment will be done using tracks reconstructed offline.

An additional thin kapton screen with a thin conductive layer will be used to surround L00. The screen can be attached to L00 or it could be installed on the inside of SVXII. The screen can also serve to protect SVXII during beam pipe installation.

The installation of the beam pipe with LO0 attached would follow procedures similar to those already planned in the absence of L00. The clearance between L00 and SVXII is adequate for installation. We anticipate no difficulties as long as the beam pipe is not accidentally struck. Special precautions will be taken to avoid such accidental deflections during installation. After installation the pipe can be rigidly supported at a number of points. Basic analyses of several possible support configurations have shown that it should be possible to sufficiently damp any movement caused by a reasonable deflection of one end of the beam pipe. 


\subsection{Alignment and Position Monitoring}

The precision with which L00 will measure track positions far exceeds the precision by which the sensors can be positioned within SVXII. Although the position of L00 sensors relative to one another can be measured using a coordinate measuring machine with a precision approaching the detector resolution (as mentioned above), no such measurements are possible once the detector is inserted into SVXII. With the placement of LOO determined entirely by a relatively imprecise installation procedure, it becomes critical that track data alone be sufficient to determine the detector alignment to a precision comparable to the intrinsic resolution.

The procedure and expected precision for a track-based alignment of L00 is the same as that for any other layer in the detector. The success of track-based alignments of SVX and SVX' in Run I suggests that similar algorithms will succed for the Run II silicon detectors. Indeed, for SVXII, the larger detector overlaps, better initial alignment and significantly better $z$ resolution than was available for the Run I detectors holds open the prospect of a greatly improved outcome. There are, however, several significant differences between L00 and the other layers in SVXII that may influence the result and merit some discussion: the dissimilar wedge geometry; the unaligned initial state; and the mechanical independence of SVXII and LOO.

The azimuthal size of L00 modules will be larger than that of wedges in SVXII. This difference has at least two consequences. First, tracks that pass through a single L00 sensor will illuminate more than a single wedge in SVXII. Second, the range of incidence angles (in azimuth) on the detector will be larger than it is for detectors in SVXII. At best, both of these differences improve the determination of detector alignment, and at worst, make no difference. In general, elements that link adjacent SVXII wedges together will help to reduce relative misalignments. The persistence of significant wedge-to-wedge misalignments in SVX and SVX' were due in part to the lack of such wedge-spanning detector elements. Initially, the complication of linking wedges together can be ignored, in which case we recover the simple single ladder alignment problem. Similarly, increasing the range of incidence angles improves the sensitivity to misalignments that change the radius of the sensors (including rotations).

After installation, it is conceivable that the position of strips within LOO relative to those in SVXII may be more than $200 \mu \mathrm{m}$ from nominal. Such a large misalignment may significantly hamper proper association of hits within L00 to tracks through the rest of the silicon. The solution to this problem is to start with a sample of low multiplicity events in which the hit associations can be made with a reasonable degree of accuracy. A small sample of such events, even if reconstructed by hand, should suffice to establish the alignment at the level of $\pm 30 \mu \mathrm{m}$ or better. Although misalignments at this level may affect the pattern recognition, experience with SVX and SVX', which were aligned to no better than 20 or $30 \mu \mathrm{m}$ initially, shows that the pattern recognition finds the 
majority of tracks in sparse events with sufficient accuracy to allow alignment via an automated algorithm.

The mechanical independence of L00 from SVXII and the accompanying possibility of relative motion of the two detectors is clearly a problem that must be overcome if the alignment is to be stable (and hence useful). The SVXII spacetube and the ISL spaceframe are designed to isolate the detectors from incidental forces that may develop during the installation process, handling of cables, etc. while the SVXII spacetube is mounted in a stress-free state in the ISL. These two detectors are therefore both well isolated from external mechanical effects. In contrast, LOO is connected directly to the beam pipe opening the possibility of it being affected by incidental forces, so the effect of such forces must be minimized.

In addition, the beam pipe is in turn connected to the SVXII spacetube, the ISL spaceframe and a number of other detector elements. This could compromise the mechanical isolation of those systems, thereby allowing incidental forces to move the other silicon detectors as well. Also, it is possible temperature dependent effects, mechanical deflections coupled to the beam pipe, or other time dependent forces could alter the detector position on a time scale sufficiently short that the data collected during any single position state proves inadequate to determine the alignment.

The solution to these problems is to design the beam pipe support to decouple motions induced at the end of the pipe from those at the center. In order to prevent movement of the ISL, the forces transmitted to the pipe at the lowprofile flanges must be transferred directly to the end of the ISL extension tube, and thereby to the COT endplate. Care should be exercised to ensure that any vibrations transmitted to the corrugated beam pipe be damped prior to reaching the Be pipe. Foam wadding, or other suitable damping material placed around the beam pipe in the $3^{\circ}$ hole would likely suffice for this purpose. We currently possess quite accurate mathematical models for the behavior of the spaceframe and spacetube. These tools can be used to test and develop various design concepts and help define the required specifications for the beam pipe mounting system. Preliminary analyses indicate that good vibration isolation can be obtained. 


\section{Chapter 3}

\section{Technical Description of Time of Flight}

\subsection{Overview}

Virtually all other experiments dedicated to the study of $B$-physics employ some type of particle identification, whether it be Time-of-Flight, $d E / d x$, Cerenkov devices or a combination of these. A Time-of-Flight detector is the simplest and most cost effective way to provide CDF II with the ability to identify $B$-decay products over a useful fraction of their momentum spectrum. Based on resolutions measured in cosmic ray tests we believe that an average timing resolution of 100 ps, obtained from a single scintillator bar, is a reasonable goal. This is also the resolution expected from a similar TOF system being installed in the BELLE detector at KEK. Figure 3.1 shows that this resolution corresponds to roughly $2 \sigma \mathrm{K} / \pi$ separation for $p<1.6 \mathrm{GeV} / \mathrm{c}$.

The proposed Time-of-Flight system design builds on several years of R\&D. The R\&D included constructing and operating a cosmic ray test stand located in B0[21] and performing a successful 5\% "full-scale" TOF system test in the CDF detector at the end of the collider running period in 1995, known as the Run Ic test[22]. The cosmic ray studies provided the essential design parameters of the Run Ic test. This test, described in more detail in References [23, 24], confirmed the viability of the design being proposed for the full system. The test also demonstrated that the time at which an individual $p \bar{p}$ collision occurred, called $t_{0}$, could be determined from the tracks in the event, and that the "hit" occupancy from neutrons and "back splash" from the electromagnetic calorimeter is not a problem. Based on the installation experience for the Run Ic test, an improved cable plant is planned for Run II.

Several design modifications came out of the experience gained from building and analyzing actual TOF data from $p \bar{p}$ collisions and this experience has now been applied to the proposed TOF system for Run II. These include using higher gain photomultipliers to offset the larger than expected gain reduction observed 


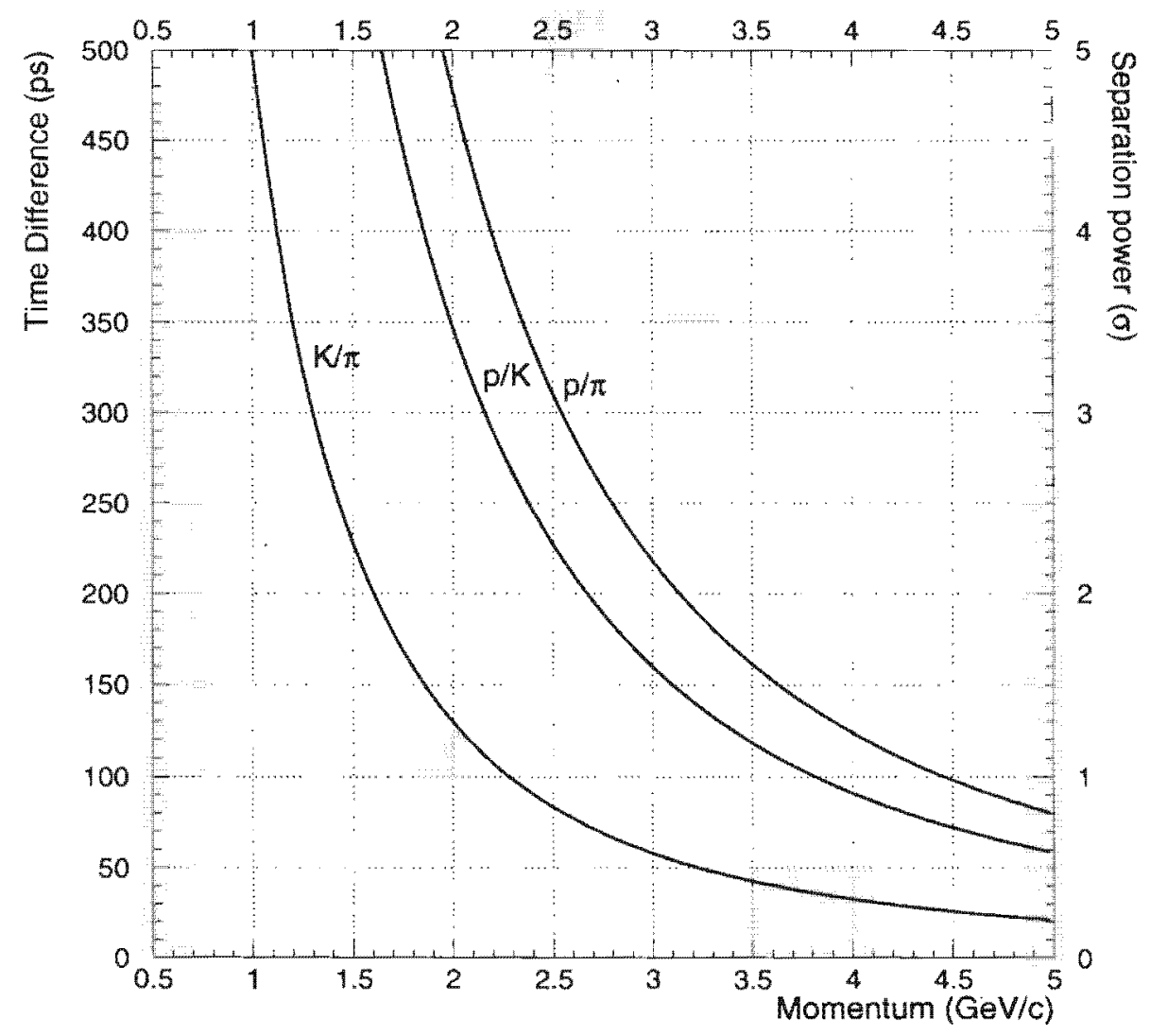

Figure 3.1: Time difference as a function of momentum between $K / \pi, p / \pi$ and $K / p$ traversing a distance of $140 \mathrm{~cm}$, expressed in ps and separation power, assuming a resolution of $100 \mathrm{ps}$. 
by operating the tubes inside the CDF solenoid and a simpler discriminator design (leading edge rather than constant fraction) that saves space and reduces cost. The signal and high voltage cables used in the test also proved to be stiff and consequently several channels were noisy due to bad connections.

More recently, another cosmic ray test stand has been developed to refine some of the issues in the design. The single main system area not addressed in the test was the timing electronics, since commercial TDC's and ADC's were used. We have prototyped the clock distribution system and a prototype Timeto-Amplitude Converter (TAC) circuit has been modeled in SPICE and a single channel has been built and tested. Based on this prior and present work, we feel confident that the proposed TOF system should reach $\sim 100$ ps single-bar resolution and will have a high efficiency and acceptable occupancy for operation even at TeV33.

The proposed TOF system consists of 216 bars of scintillator, $3 \mathrm{~m}$ in length, arranged in a cylinder around the main CDF II tracker, the COT. The location of the TOF is illustrated in Figure 3.2. Mounted on each end of every bar is a fine mesh 19-stage 1.5 inch Hamamatsu photomultiplier tube. Connected to each tube is a base that provides the high voltage divider chain and a preamplifier to increase the signal size. The signals from the preamplifier are driven differentially to a leading edge discriminator and the TOF Front End Electronics card (TOFFEE) which contains a single timing channel. This circuit includes a timeto-amplitude converter (TAC) and an ADC that digitizes the resulting voltage. The readout makes use of the CDF analog memory module (ADMEM) which normally contains calorimeter front-end (CAFE) cards, mounted as daughter boards. Each ADMEM board would have its CAFE cards replaced by 18 TOFFEE cards. In this way we will obtain timing measurements while utilizing the already developed VME and trigger interfaces. A very stable $(<25 \mathrm{ps})$ TOF clock distribution system is used to generate the start-pulse for each TAC. The pulse height, necessary for a time-walk correction, is measured with a simplified CDF II CAFE card (called the DECAF card) that is also mounted as a daughter card on a separate ADMEM board.

\subsection{Scintillator and PMT Assembly}

There is about $4.7 \mathrm{~cm}$ of radial space available between the outer can of the COT and the cryostat. This space would be occupied by bars of scintillator $4 \mathrm{~cm}$ thick and $3 \mathrm{~m}$ long, to cover the length of the COT. The optimal width of the bars is related to issues of timing resolution and counter occupancy and was determined to also be about $4 \mathrm{~cm}$. Thus, 216 bars of scintillator would be required for full azimuthal coverage at a radius of about $140 \mathrm{~cm}$ from the beam axis. Bars with a trapezoidal cross section would be used to minimize the space in cracks between them.

The scintillator for the proposed system would be Bicron 408, which has 


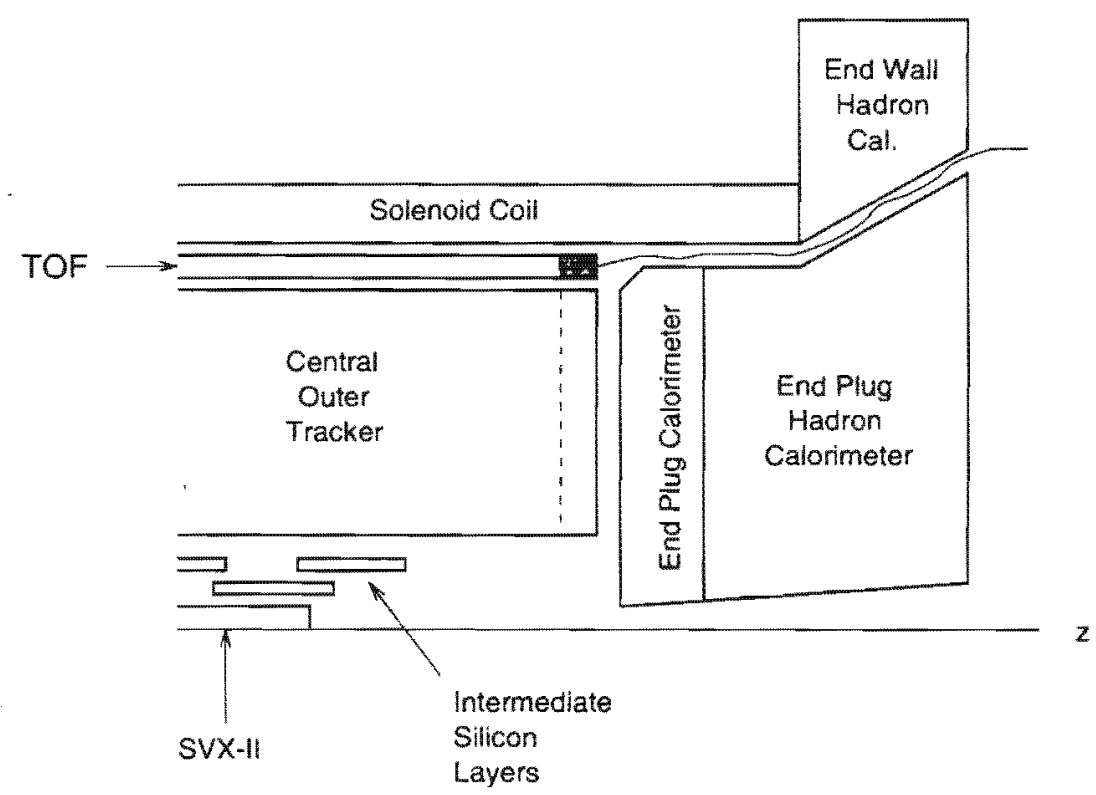

Figure 3.2: Side view of CDF II showing the location of the Time-of-Flight subdetector.

both a short rise-time and a long attenuation length of $\sim 380 \mathrm{~cm}$. As shown in figure 3.3, the photomultiplier tubes are attached to these bars via Winston cone light guides to optimize the timing resolution. The photomultipliers would be

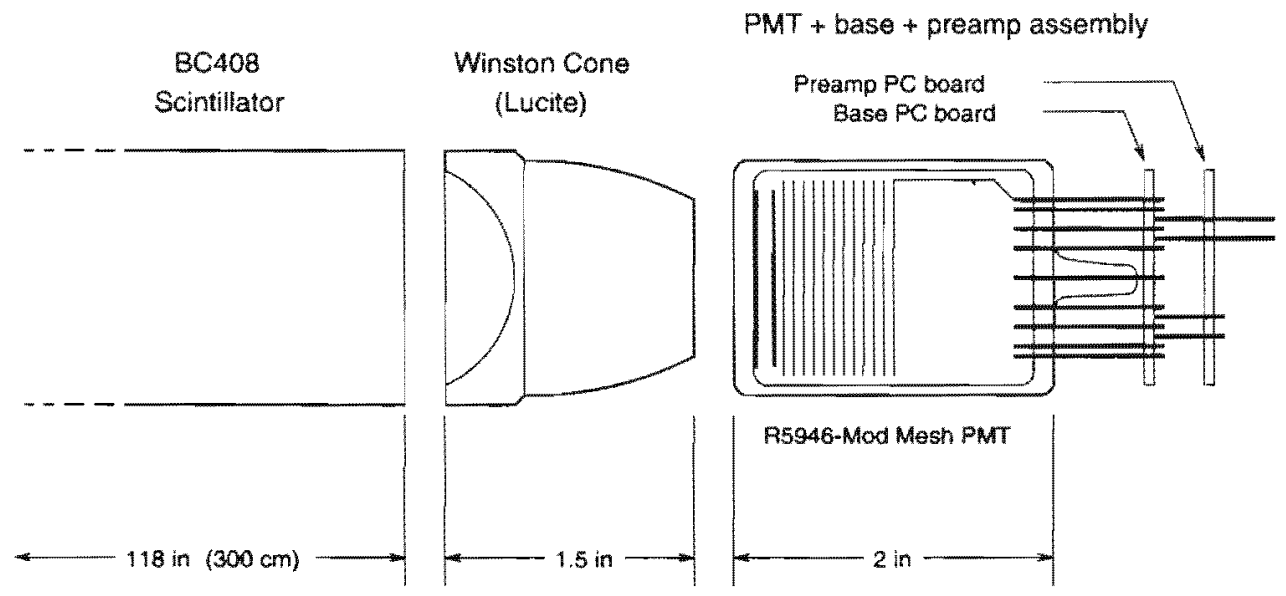

Figure 3.3: Arrangement of the scintillator, Winston cone and PMT assembly. This also shows the printed circuit boards for the PMT base and the preamplifier.

Hamamatsu R5946-Mod tubes or an equivalent, if available. These are 1.5 inch fine mesh tubes with 19 dynode stages which will provide sufficient gain in the magnetic field. We intend to purchase tubes rated for operation with grounded cathodes, ie. positive high voltage. This is because these tubes are not required to have a low leakage current across the glass face which results in a significant reduction in cost. Cables from each end of a bar are brought out to the same 
end of the detector and interface to the same electronics module. Not only does this simplify the calibration procedure, but it also makes possible the eventual integration of TOF into a Level 2 mass trigger. This would require $z$-information obtained from the difference in time measured at opposite ends of a bar.

\subsection{Electronics}

\subsubsection{PMT base}

The PMT base design is similar to the one used to build bases for the 16 stage tubes used in the Run Ic test. It has been modified to accommodate 19 dynode stages of the R5946-Mod tube and includes a DC blocking capacitor at the anode. As has been done in the past, the base will be constructed on a small, round printed circuit board using surface mount components. Each base will draw about $100 \mu \mathrm{A}$ of current at its operating voltage. Based on our previous experience building similar bases, we anticipate no problems in their construction or their long term operation.

\subsubsection{PMT preamplifier}

The preamplifier for the PMT's will also be built on a small printed circuit board and mounted directly behind the PMT base. The circuit, shown in figure 3.4, is a classic common base amplifier configuration with emitter followers to drive the base of the shaper/driver output transistor. The circuit shown was built and used for 16 stage R5946 mesh tubes with good results. It has a wide dynamic range and has proven to be very reliable and robust. We propose to modify this design slightly by adding a PNP transistor at the output stage to drive a differential signal on a shielded twisted pair cable. This will provide additional common mode rejection and the mutual inductance between the two conductors will reduce the cable dispersion.

The preamplifiers require low voltage power and a control line which can be used to attenuate the gain. This attenuation will be necessary if they are to be tested with the CDF magnet off. It will be convenient to bring the low voltage and control lines into the detector from the front-end electronics crates via the transition boards which receive the PMT pulse signals. So as to remain isolated from the crate power supply, separate low voltage supplies would be mounted near the front-end electronics crates specifically for supplying power to the preamplifiers.

\subsubsection{High Voltage}

Mesh PMT's generally have very stable gains even in a high magnetic field. Since timing measurements are the primary interest, with pulse height information 


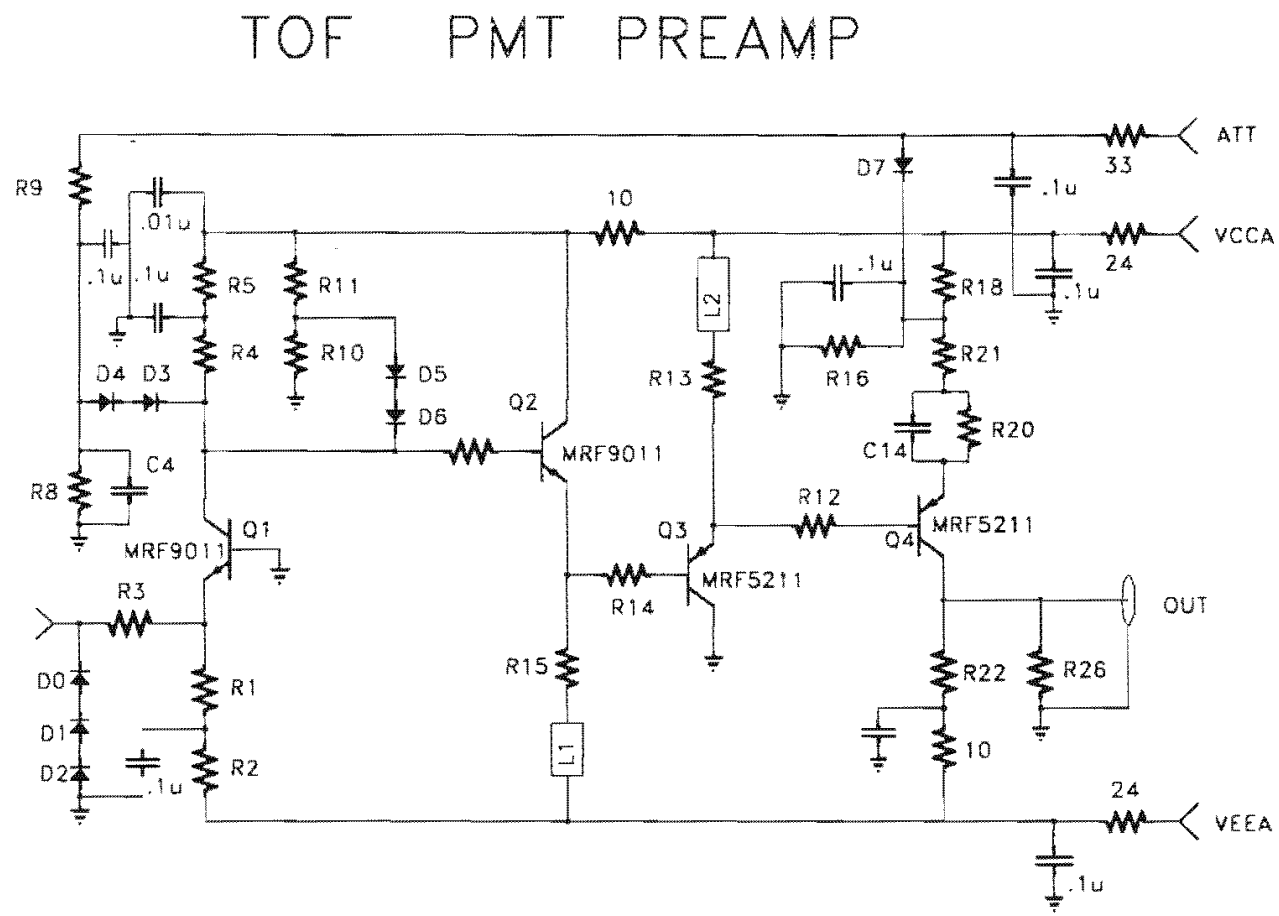

Figure 3.4: Schematic of the TOF preamplifier used in the 20 bar test.

used mainly for offline walk corrections, we will not require the ability to control the gains of individual PMT's during a run. However, the gains on individual mesh PMT's can vary widely. Although we do not need fine control of the gains we will need to set the high voltage on each tube separately so that the gains are approximately equalized and to avoid possible saturation of the preamplifiers for the largest pulses. The most cost effective means to achieve this is through a resistor-zener network powered from a bulk high voltage supply. The total current needed for the entire system would be less than $50 \mathrm{~mA}$ with PMT's operated at 2000 volts.

A block diagram for this high voltage distribution system is shown in figure 3.5. Since the current draw on each base will be known, the resistors at the last stage can be chosen to fix the predetermined PMT gains. This requires measuring the gains of all PMT's in a magnetic field prior to installation and can be done using a 1.4 Tesla test magnet located at $\mathrm{B} 0$. The performance will not depend strongly on the actual gains but optimal performance could be achieved by adjusting the resistor values during short accesses to the experimental area. 


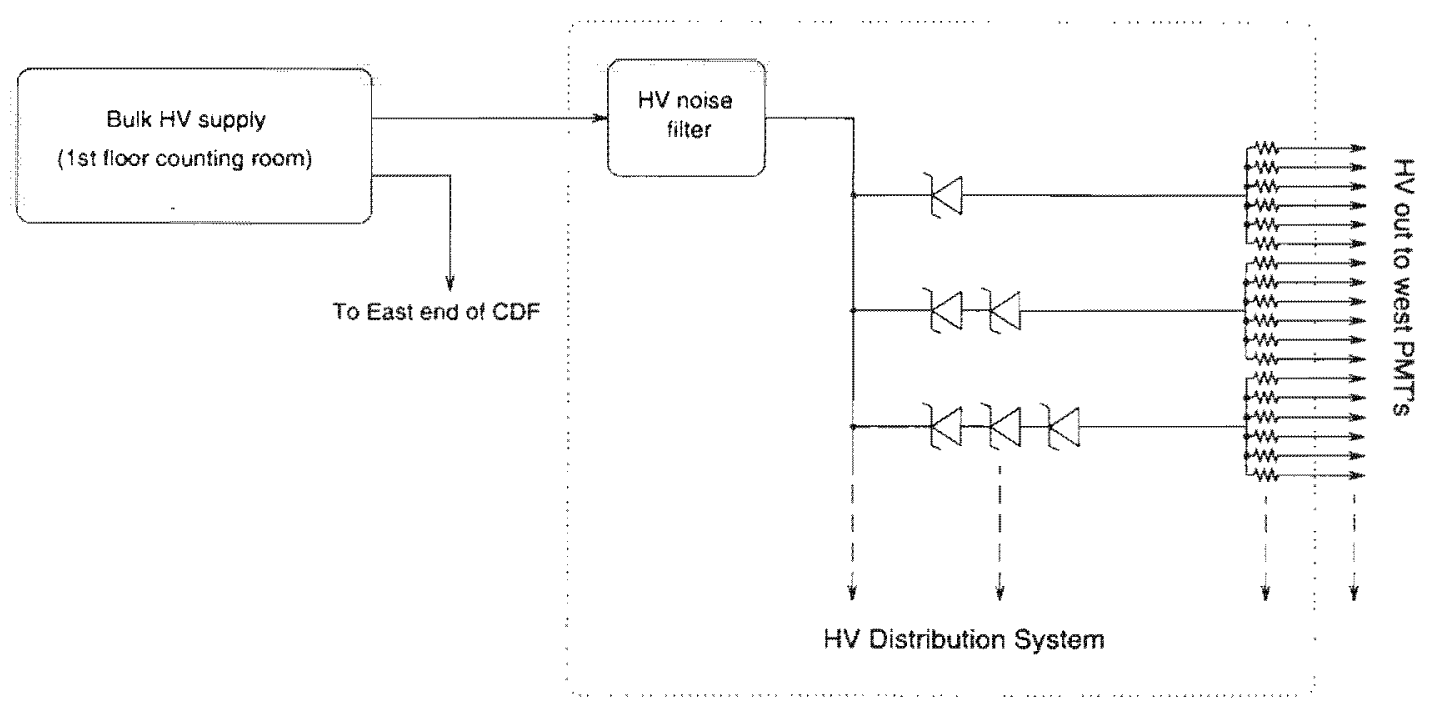

Figure 3.5: High voltage distribution system.

\subsubsection{TDC Design}

The front-end electronics for TOF are required to provide precise timing information and measurements of pulse heights. We desire a timing stability in the electronics better than about 25 ps so that the photon statistics remains the dominant factor limiting the timing resolution of a single PMT. This level of timing stability is an order of magnitude beyond what is required in most other CDF II electronics. This level of precision for a TOF system has been achieved by the CLEO collaboration[25].

We intend to make use of the front-end electronics developed for CDF's electromagnetic calorimeter with appropriate modifications where necessary. The calorimeter front-end electronics digitizes PMT pulse height information using 20 channel ADMEM modules. These VME modules receive the PMT signals via transition boards on the back of the crate and attach via a connector isolated from the VME bus backplane. The ADC electronics for each channel is located on a removable CAFE daughter board. The ADMEM board also provides the logic necessary to buffer events in a pipeline while awaiting a trigger. Since this electronics infrastructure has already been developed, we intend to make use of it as the basis for our TDC measurements.

The front-end electronics for TOF will be located space already allocated in eight Endwall VME crates mounted on the detector. Figure 3.6 shows the electronics components required for a single crate. This figure shows the transition board used to receive the PMT pulses and to distribute low voltage power to the preamplifiers. These also provide buffering of the PMT pulses so that their pulse height can be measured via ADMEM modules in adjacent slots.

To provide time measurements, the removable CAFE cards are replaced by the TOFFEE cards containing the TAC and ADC. With a smallest count of 
25 ps, a 12 bit ADC will be more than sufficient to cover the required dynamic range of $\sim 50$ ns. A candidate ADC is the Analog Devices AD9042 chip which is similar to the 10 bit AD876 chip used on the CAFE modules. Each TDC digitizes the time between a common start clock pulse and the pulse from the discriminator which is located on the transition board.

The proposed TAC design, shown in figure 3.7 , is intended to have minimal sensitivity to noise pickup and leakage current on the timing capacitors. The differential ECL start signal inhibits the charging of the timing capacitors and initiates a linear ramp on one of them. The difference in voltage between the two capacitors is then proportional to time, with any droop due to leakage cancelled to first order. In addition, any noise present would be picked up equally on both capacitors and would cancel in the difference. This motivates operating the TDC's in a common start mode, where synchronous switching noise will be picked up symmetrically on each capacitor at the start of the timing cycle. The differential ECL stop signal from the discriminator halts the linear ramp and initiates sampling of the voltage difference across the capacitors. A prototype TAC circuit has been constructed and tested. The next step is to interface it with the $\mathrm{ADC}$ and integrate several channels into electronics for a cosmic ray test setup.

The bunch length in the Tevatron is rather long $(\sim 30 \mathrm{~cm})$ and the times at which $p \bar{p}$ interactions occur will be distributed over a range of a few nanoseconds in each event. To provide precise time-of-flight measurements, we need to measure the time, $t_{0}$, at which each interaction occurred. This procedure, described in detail in section 3.6.2, requires that all 432 TDCs in the front-end electronics receive the start pulse to within the desired $25 \mathrm{ps}$ resolution. The modifications to the ADMEM board are required to achieve and maintain this level of precision.

To achieve these goals, a new connector is required on the front panel of the ADMEM board to receive a precision differential ECL clock signal. Additional traces are required on the ADMEM board to fan out this differential signal to each of the TOFFEE cards. Although this requires the placement of only 4 new IC's for the on-board clock fanout, the routing of the traces is critical and will require careful board layout. Although the Fermilab EE support is not required to make these board modifications, their past experience with and knowledge of the existing ADMEM board design will be valuable. This motivates performing these changes in board layout at Fermilab where reasonably close contact with the EE staff who worked on the original ADMEM board can be maintained.

Because of the stringent timing requirements of the electronics, time dependent changes due to temperature drifts, ground voltage shifts or aging of the components must be anticipated. It is essential to include ways of measuring these effects in the design of the electronics. Thus, a calibration clock located on the transition board and driven by a temperature stabilized quartz oscillator is included in the design. This provides a fast and easy way to determine the slope of the amplitude vs time dependence of the TAC electronics which can be 
performed as often as is required. The ability to monitor the temperature at key points on the TOFFEE cards is also desirable as it could allow the correlation of timing and temperature changes to be observed and accounted for. In general, we can provide insurance that the electronics will perform with the desired level of precision by providing as many means of calibration and monitoring as possible.

To simply apply an offline correction for discriminator walk, the full precision and dynamic range provided by the CDF II ADMEM board is not required. To reduce the cost of the electronics, we will also build simpler DECAF cards and use them instead of the CAFE cards in ADMEM modules used for pulse-height measurements. This is a simplification of the TOFFEE card design since it does not include the components for the TAC.

\subsubsection{Clock Distribution}

To achieve the required timing precision, a common start signal must be sent to all TDC channels with a stability of $<25$ ps. A clock signal would be derived from the Tevatron RF and a dedicated fanout system for TOF would be required to achieve and maintain this stability. The TOF clock fanout system would be an order of magnitude more stable than the clock distribution used for the synchronization of CDF electronics for the trigger and event readout [26] but would also be considerably simpler.

All clock signals would be differential ECL and would be sent along shielded twisted pair cables from a central fanout point to each crate and to each TDC module within each crate. This clock distribution scheme is based on Motorola's ECLinPS series of integrated circuits. The system- and crate-level fanout would each be driven by MC100E111 chips which provide a 1:9 fanout of a differential ECL signal. This chip has a very small channel-to-channel skew of typically $<25$ ps which implies a channel-to-channel jitter of much less than this.

Although the event-to-event jitter would be very small, it is important to measure the actual propagation delay from the central fanout location to each of the crates. This will allow longer term drifts to be monitored and included in offline calibrations. This will be achieved by receiving the clock pulse in each crate and, in addition to distributing it to the TDC modules, returning it along a separate clock cable to the central distribution point. A TDC in the central clock fanout module would then measure the round-trip time which would include all delay effects external to the electronics crates.

One important cause of changes in propagation delay is the temperature of the clock cable. Thus, we propose an additional cross check whereby the temperature along each cable is measured at eight points with which we could correlate measured changes in the round-trip propagation delay. Even larger changes in signal speed are caused by thermal gradients across the dielectric in the cable and we intend to minimize these by wrapping the clock cable, the 
returned clock signal cable and the temperature sensors in mylar insulation.

We have built a prototype clock distribution system which drives a differential ECL signal on a 40 foot cable. The propagation delay was measured with a LeCroy 11 bit TDC with 50 ps resolution. Although the spread of propagation delays was much less than this resolution, we measured it by introducing an additional delay proportional to an analog control signal. By varying this voltage the delay distribution could be scanned across 50 ps count boundaries allowing its width to be be measured. In this way we determined that the instantaneous jitter associated with both the clock fanout electronics, the cable and the TDC was $\mathcal{O}(3 \mathrm{ps})$.

\subsection{Calibration and Monitoring}

To maintain precise timing resolution it will be crucial to monitor the key elements of the TOF system so that changes in operating conditions can be detected and the appropriate calibration corrections applied to the data. Since TOF information will be an important element in the analysis of CP violation and $B_{3}^{0}$ mixing measurements, it is important to minimize the time required to commission and calibrate the system so that these key measurements will be made as quickly and efficiently as possible.

As described in sections 3.3 .4 and 3.3 .5 we intend to integrate some calibram tion measures into the design of the electronics. The crystal oscillator driven clock generator on the TDC transition boards allows the slope of each TDC channel to be determined as often as will be necessary. This makes it easy for any changes in timing due to drifts in the ground voltage or crate temperature to be detected and calibrated out. It will also be useful to correlate any such changes with observed changes in the temperature at key points on the electronics boards and along the clock distribution cables.

We also consider two methods by which we could inject light directly into the scintillator. The first is via LED's located at the ends of the bars in the PMT mounting assembly. Although not useful for timing calibration, the information obtained from pulsing the LED's is valuable for detecting bad channels or cabling errors and would speed up the commissioning phase of the TOF system. The LED's would also provide an additional way to monitor long term changes in the attenuation length of the scintillator. This system is particularly attractive since it can be implemented with almost negligible additional cost.

To study as many aspects of the system as possible in a well controlled way, we also propose a laser calibration system. Light from a nitrogen laser would be brought into the detector along glass fibers and injected into the bars to excite the scintillator. The laser would also excite a piece of scintillator attached to a PMT outside the detector to provide a reference time. The time difference between TOF channels and the reference time would allow the timing resolution on individual channels to be measured and monitored. This would 
provide an important way to validate changes in calibration constants without relying exclusively on data recorded with physics triggers. CDF has already purchased a Nitrogen laser for TOF R\&D work which can be used for this system.

\subsection{Installation and Integration}

To prevent loading and possible deflection of the COT endplates, we would support the scintillator for TOF from mounting hardware attached to the cryostat. In the current CDF II upgrade schedule, the CTC used in Run-I will be removed at the end of August 1999 which will allow free access to the inside of the cryostat. We would then perform a survey of the radius of the inner surface at several points and locate the positions of welded seams in the cryostat. If it is found that the cryostat is more than $3 \mathrm{~mm}$ out-of-round, adjustments to the mounting hardware dimensions could be made at this point.

\subsubsection{Mounting Scheme}

We have designed the mounting hardware such that it can be partially disassembled to provide maximum radial space for the insertion of the COT, and then reassembled after the COT is in place. The proposed design is shown in figure 3.8 and consists of three components. A total of 24 slotted aluminum rails can be safely attached to the inner surface of the cryostat either by gluing or using spot-welded threaded aluminum studs. Extruded aluminum fins running the length of the detector are then inserted into these slots. These fins have notches at their inner radius which hold rolled aluminum shelves in position on which the scintillator rests. The mount would be attached, assembled completely, surveyed and removed in about a week. There would then be about $3.5 \mathrm{~cm}$ of radial clearance for the COT to be inserted after which the mount would be completely reassembled. The scintillator could then be inserted into the mount at any time thereafter.

\subsubsection{Cable Routing}

The inner subdetectors will route their cables radially outward to slots in the $30^{\circ}$ crack between the plug calorimeter and the central electromagnetic calorimeter. The natural cabling scheme is for the outermost subdetectors to be cabled first, having their cables covered by successive layers of cables from the inner subdetectors. Thus, it is highly desirable to have all cables in place prior to the cabling of the COT. As we will have only 216 channels per side, the time required to fully dress and route the TOF cables will be short compared with cabling the COT, ISL and SVXII subdetectors and should not interfere with their installation schedule. 
The cables to be routed include the high voltage cables for the PMT's, low voltage, signal and control lines for the preamplifiers as well as control lines for pulsing the LED's and fibers for the laser calibration system. Some of the low voltage and control lines can be distributed from inside the detector and will not require routing separate cables for each channel. As previously discussed, the signal cables from PMT's at both ends of a scintillator bar are brought out to the same side of the detector but this is not necessary for the high voltage or preamplifier power and control signals.

\subsection{Event Reconstruction}

Many aspects of event reconstruction with this TOF system have been studied using a fast Monte Carlo parametrization[27]. This assumed that the timing resolution varied linearly with the distance, $d$, from the phototube at which a track entered the scintillator:

$$
\sigma_{t}=(100 \mathrm{ps})+(0.4 \mathrm{ps} / \mathrm{cm}) \cdot d
$$

and is motivated from cosmic ray tests. Event reconstruction proceeds by first associating PMT hits with tracks and then determining the times ( $t_{0}$ 's) at which $p \bar{p}$ interactions occurred in the event. Several $p \bar{p}$ interactions can occur in a single bunch crossing and it will be important to determine the production times of each. These times must be known much more precisely than the overall event $t_{0}$ used, for example, by the COT to measure drift times. Ultimately, each interaction will have its $t_{0}$ measured to approximately 30 ps which will in no way limit the performance of TOF for particle identification. Given the production time, each track from a $p \bar{p}$ interaction vertex will have its time-offlight determined.

\subsubsection{Association of tracks with PMT's}

Some events may have as many as $20 \%$ of the activated TOF counters hit by more than one track in Run II and as many as $30 \%$ for luminosities of $10^{33} \mathrm{~cm}^{-2} \mathrm{~s}^{-1}$ in possible future Tevatron runs. However, the way this influences the ability to perform particle identification depends on how information available from the TOF system is used. In particular, the particle identification efficiency depends on the algorithm used to associate tracks with PMT's. We have considered two approaches and have studied how they perform at high luminosities.

A common algorithm used to determine whether both PMT's on a bar are associated with a track is to compare the extrapolated $z$-position of the track, determined using information from the tracking chambers, to the $z$-coordinate determined from the time difference:

$$
z=\frac{1}{2}\left(T_{L}-T_{R}\right) \cdot s_{\text {bar }}
$$


where $T_{L}$ and $T_{R}$ are the times measured at left and right ends of the bar and $s_{\text {bar }}$ is the speed of light propagation in the bar. We refer to this method as the " $T_{L}-T_{R}$ " algorithm and consider a track to be associated to both PMT's on a bar if the extrapolated and measured $z$-position agree to within $2 \sigma$. The efficiency with which this algorithm associates hits with tracks and the frequency with which hits are incorrectly assigned to a track are shown in figure 3.9.

This algorithm does not make optimal use of the available information since the PMT's on bars which are hit by multiple tracks will not, in general, be associated with any of them. An alternative algorithm has been considered in which a PMT is associated with a track only if no other tracks hit the bar at $z$-positions between the track in question and the PMT. Since the distributions of $t_{0}$ and time-of-flight for tracks are typically shorter than the time required for light to propagate to the ends of a bar, this algorithm usually makes the association correctly. We refer to this as the "closest track" algorithm and the efficiency with which it associates tracks with PMT's and its misassociation frequency are shown in figure 3.10 .

\subsubsection{Determination of Interaction $t_{0}$ 's}

In general, we may need to determine the $z$-coordinate and $t_{0}$ of each interaction in the event. The problem of finding $t_{0}$ for each interaction in the event will reduce to applying a clustering algorithm to tracks in $z$ and $t_{0}$ and determining the mean $t_{0}$ for each interaction separately. However, for analyses in which a $B$ decay is fully reconstructed it will be sufficient to identify only those tracks associated with its primary interaction vertex. We have studied the details of this latter problem.

The average production time for the four final state tracks in a $B^{0} \rightarrow J / \psi K_{\mathrm{S}}^{0}$ decay $\left(\mu^{+} \mu^{-} \pi^{+} \pi^{-}\right)$already determines the $t_{0}$ of the event with a resolution of about 63 ps. This is sufficient to identify tracks in the event which have production times consistent with the $t_{0}$ of the $J / \psi K_{\mathrm{S}}^{0}$ primary vertex. These tracks are used in the $t_{0}$ fit if their transverse and longitudinal impact parameters are within $0.5 \mathrm{~cm}$ and $1.0 \mathrm{~cm}$ of the $J / \psi$ vertex, respectively, and if their production time, under any of the $\pi, K$ or $p$ hypotheses, is within $5 \sigma$ of the $J / \psi K_{S}^{0} t_{0}$ estimate.

Figure 3.11 shows the $t_{0}$ distributions for both the $J / \psi K_{\mathrm{S}}^{0}$ alone and for the full event, obtained by fitting $t_{0}$ using tracks associated with the $J / \psi K_{\mathrm{S}}^{0}$ production vertex. The $t_{0}$ resolution is found to be about $33 \mathrm{ps}$ and has a negligible effect on particle identification. This figure also shows the distribution of the number of tracks used in the fit. This has a mean of 10 , in addition to the $J / \psi$ and $K_{\mathrm{S}}^{0}$ tracks.

The fit is performed by first computing the expected production times for 
each track under assumed particle hypotheses:

$$
t_{i}^{h}=T_{i}-D / c \sqrt{1+m_{h}^{2} / p^{2}}
$$

where $T_{i}$ is the time recorded by the TOF counters, $D$ is the path length of the track from the production point to the counter and $p$ is its momentum.

Then, the $t_{0}$ for the interaction is determined by maximizing the likelihood function

$$
\mathcal{L}=\frac{1}{\sqrt{2 \pi} \sigma_{t}} e^{-\left(t_{J / \psi K_{S}^{0}}-t_{0}\right)^{2} / 2 \sigma_{t}^{2}} \cdot \prod_{i}\left(f_{\pi} \mathcal{L}_{\pi}\left(t_{i}^{\pi}, \sigma_{t_{i}} ; t_{0}\right)+f_{K} \mathcal{L}_{K}\left(t_{i}^{K}, \sigma_{t_{i}} ; t_{0}\right)+f_{p} \mathcal{L}_{p}\left(t_{i}^{p}, \sigma_{t_{i}} ; t_{0}\right)\right)
$$

where $t_{J / \psi K_{S}^{0}}$ is the measured production time of the $J / \psi K_{\mathrm{S}}^{0}$ with uncertainty $\sigma_{t}, f_{h}$ are assumed particle production fractions and

$$
\mathcal{L}_{h}\left(t^{h}, \sigma_{t} ; t_{0}\right)=\frac{1}{\sqrt{2 \pi} \sigma_{t}} e^{-\left(t^{h}-t_{0}\right)^{2} / 2 \sigma_{t}^{2}}
$$

The interaction $t_{0}$ resolution roughly scales with the number of tracks from fragmentation and in the underlying event as $1 / \sqrt{N_{\text {track }}}$. Thus, there will be sufficient information available to determine $t_{0}$ as precisely as will be necessary even for, physics processes with low multiplicity final states such as $p \bar{p} \rightarrow \tilde{\tau} \overline{\tilde{\tau}}$.

This same procedure was used to determine $t_{0}$ in events recorded during the Run Ic test[23]. Because only $5 \%$ of the full system coverage was installed there was, on average, less than one track per event with which to determine $t_{0}$. Nevertheless, this algorithm was shown to be effective and we are confident that it will perform as expected for the full TOF system.

\subsubsection{Kaon Identification using TOF}

Particle identification using TOF is performed by comparing the event $t_{0}$ with the expected production time of a track, calculated using its momentum, pathlength, time-of-flight, and an assumed mass. We also calculate the uncertainty on this difference, and use it to form a $\chi^{2}$ variable for each mass hypothesis. The probability $\lambda_{i}$ that a given track is a particular particle type $i=\pi, K, p, e, \mu$ is calculated using a normalized likelihood:

$$
\lambda_{i}=\frac{f_{i} L_{i}}{\sum_{j} f_{j} L_{j}}
$$

where $L_{j}=\exp \left(-\chi_{j}^{2} / 2\right)$ and $f_{j}$ are $p_{T}$ dependent particle fractions, determined from the Monte Carlo. With real data, it will be sufficient to use estimates of these fractions using Monte Carlo, but we can also measure $f_{i}\left(p_{T}\right)$ directly using TOF.

A sample of kaons is selected by requiring $\lambda_{K}>\lambda_{K}^{\text {cut }}$. The efficiency with which real kaons are selected and the purity of the resulting sample are shown for 
a range of values of $\lambda_{K}^{\text {cut }}$ in figure 3.12. For this figure, we have used the Monte Carlo dataq sample described in Section 5.3.1. It can be seen from this figure that the "closest track" algorithm has a higher efficiency, as it must, since the PMT's it associates with tracks form a superset of those associated by the " $T_{L}-T_{R}$ " method. Nevertheless, the class of tracks which have only one associated PMT will have worse average timing resolution. Therefore, the highest purity kaon samples will generally contain tracks with which both PMT's are associated.

\subsubsection{TOF System Occupancy}

The occupancy expected in Run II will depend on the luminosity per bunch in the Tevatron. The number of additional minimum bias events which will be present for various luminosities and bunch crossing intervals is listed in table 3.1.

\begin{tabular}{|r|r|r|}
\hline $\mathcal{L}\left(\mathrm{cm}^{-2} \mathrm{~s}^{-1}\right)$ & $\tau_{\mathrm{bc}}(\mathrm{ns})$ & $\left\langle n_{\mathrm{mb}}\right\rangle$ \\
\hline \hline $.5 \times 10^{32}$ & 396 & 2 \\
$2 \times 10^{32}$ & 396 & 5 \\
$2 \times 10^{32}$ & 132 & 2 \\
$10 \times 10^{32}$ & 132 & 10 \\
\hline
\end{tabular}

Table 3.1: Expected $\left\langle n_{\mathrm{mb}}\right\rangle$ for different luminosities and times between bunch crossings.

The average counter occupancy in $B^{0} \rightarrow J / \psi K_{\mathrm{S}}^{0}$ events for these various operating scenarios is shown as a function of the mean number of additional minimum bias events, $\left\langle n_{\mathrm{mb}}\right\rangle$, in figure 3.13. These estimates are made using minimum bias events recorded in Run-I with the reconstructed tracks propagated to the positions which would be occupied by the TOF system. These additional tracks are added to the event record of Monte Carlo events in the physics studies used to quantify the enhancements provided by TOF under real operating conditions. $\mathrm{g}$

\subsection{Performance Summary of TOF R\&D}

We very briefly summarize three $R \& D$ projects for the TOF system; a cosmic ray test at $\mathrm{B} 0$, the 20 -bar test in Run Ic, and a cosmic ray test at Penn. The first data was recorded in 1995 using a cosmic ray test stand located in B0. The results from this early test indicated that outside of a magnetic field a resolution of $\sim 100$ ps was possible. A typical timing performance plot is shown in Fig. 3.14.

At the end of the Run Ib running period, a test TOF system was installed between the Central Tracking Chamber and the 1.4 Tesla superconducting solenoid 
at a mean radius of $140 \mathrm{~cm}$. The TOF system consisted of 20 bars of Bicron BC408 scintillator, each $130 \mathrm{~cm}$ in length with a cross section of $4 \times 4 \mathrm{~cm}^{2}$. To each end was fitted a small compound parabolic concentrator (Winston cone) and a 16-stage R5946 Hamamatsu fine-mesh photomultiplier. The arrangement of the scintillation bars is shown in Fig. 3.15.

The electronics chain consisted of a custom designed preamplifier, constant fraction discriminator, and commercial TDC and ADC Fastbus modules readout with the CDF data acquisition system. The CDF charged particle tracking system was used to measure the arclength of the helical track from beam collision point to the scintillation bar. In general, the charged track momentum resolution and the intersection of the charged track with the scintillation counter as determined by the tracking system had a negligible contribution to the mass error. At very low momentum, however, the contribution to the timing resolution from multiple Coulomb scattering can be significant. No attempt was made to separate the timing resolution from various CDF detector effects, since the main goal was to measure the overall performance of the system. We determined a procedure for calculating the interaction $t_{0}$ time from all tracks in an event, the timing resolution, and the particle identification performance of this system.

Fig. 3.16 shows one measure of the performance of the test system. The system subtended only about $5 \%$ of the solid angle, and so typically very few tracks per event were detected. Fig. 3.16 used one track to determine the event start time, and one track was used for the mass plot. A paper, reporting these results, has been submitted to NIM and here we summarize some of the results and conclusions from the test.

The test system performance can be determined in a number of ways and used to estimate the performance expected for a full system. We measured a flight time resolution, averaged over all tubes, of $220-250$ ps. In this analysis there was usually only one charged track available to define $t_{0}$. In a full TOF system we would typically have 10 charged tracks from which $t_{0}$ could be determined. Correcting for this fact, the resolution of a full system would be 155-180 ps. The best performing tubes indicate a single bar resolution of $\sim 100-125$ ps is attainable.

We have performed tests and simulations to determine the cause of the lower than anticipated average PMT timing resolution. A combination of broken glue joints and performing an average walk correction (due to limited statistics) rather than an individual tube walk correction accounts for about 40-50 ps degradation in resolution. In addition, smaller than expected signals from the PMTs in the CDF magnetic field coupled with the constant fraction discriminator design is also a potential cause of the lower resolution. This suggests that higher gain tubes will perform better. It is possible to purchase a 19-stage 1.5 inch diameter tube from Hamamatsu, which will allow greater gain in the field. Care will also be required to ensure good glue joints, if used. We are investigating a different procedure for mounting the PMT assemblies which should relieve some of the stress at the optical interfaces. In summary, this small test system has been 
extremely valuable in guiding us to extract the best timing resolution from the "full" TOF system.

More recently, a TOF test stand has been developed at the University of Pennsylvania. The test stand is being used to explore a number of improvements to the TOF design. As a measure of the performance being obtained from this setup, we simply present the results of a recent cosmic ray data run in Fig. 3.17 After modifications to the electronics chain and better tube-by-tube corrections, it can be seen that resolutions well below 100 ps have been achieved. It should be noted that other groups, most notably the BELLE TOF group of H. Kichimi et al. have also achieved very good resolutions in their beam tests. 


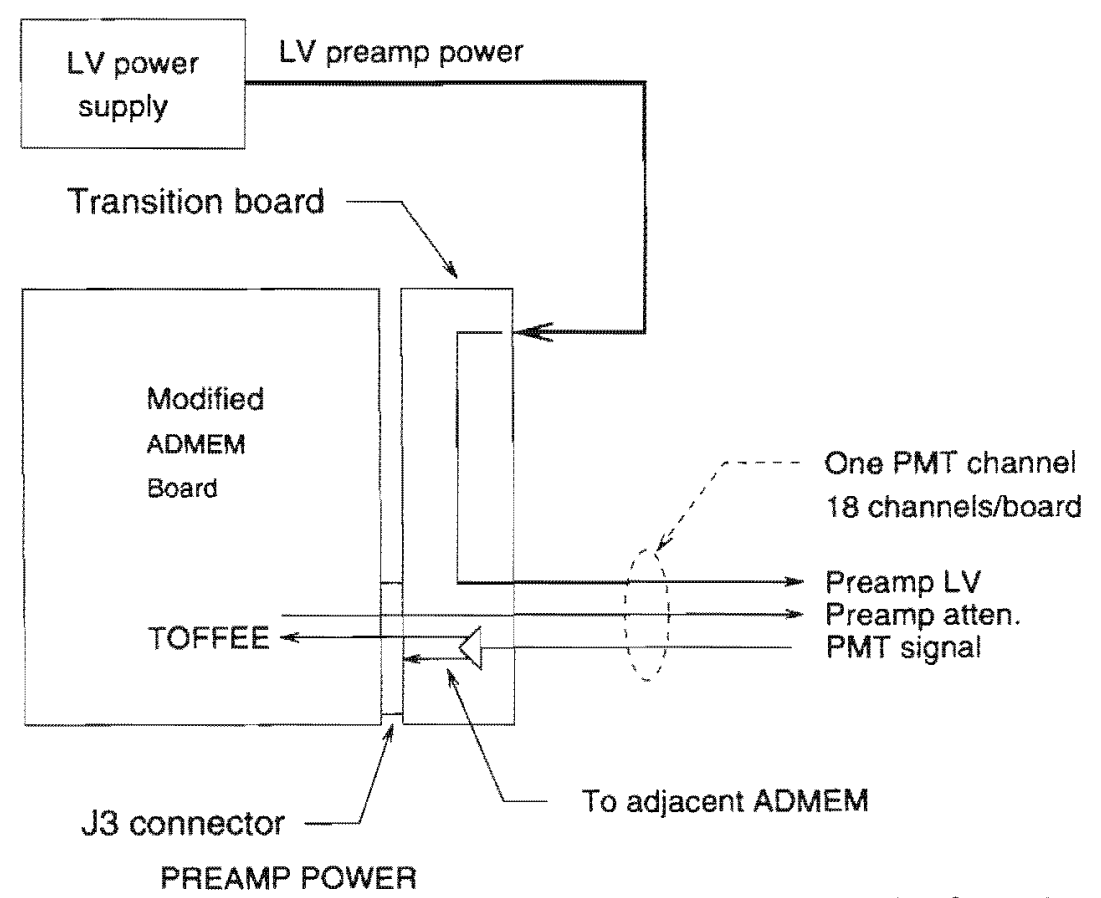

PREAMP POWEA

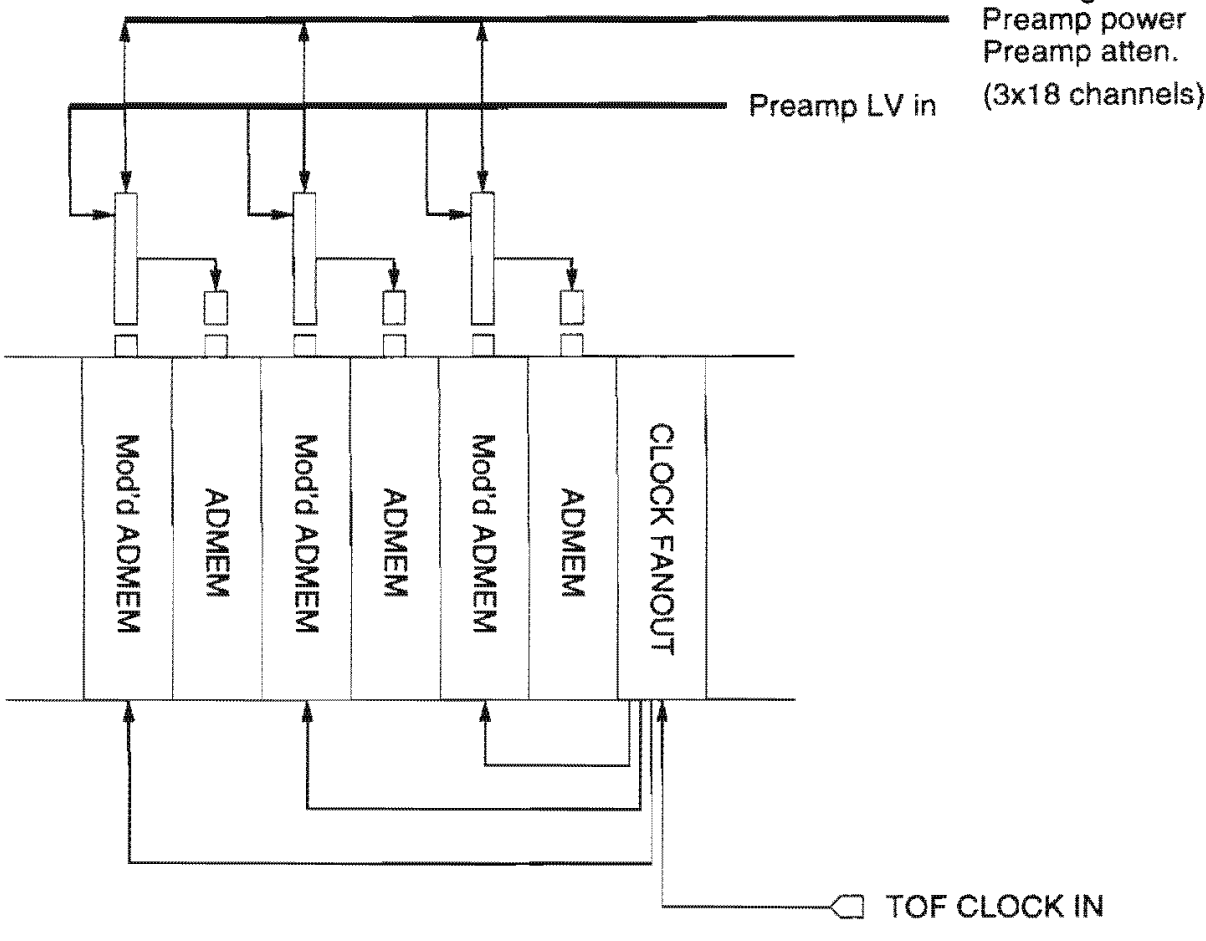

Figure 3.6: Components in the TOF electronics for a single crate. 


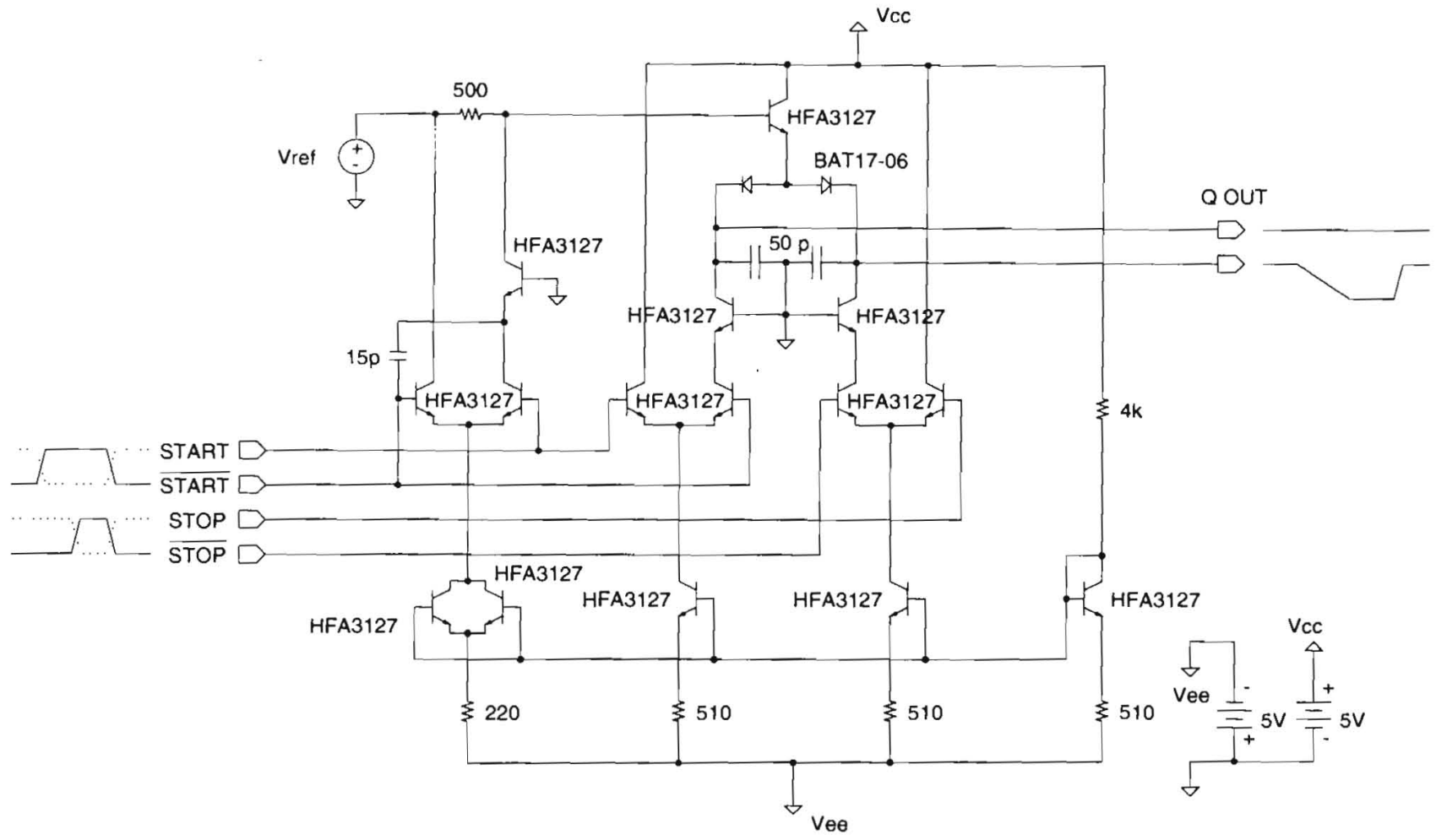

Figure 3.7: Time to Amplitude Converter (TAC) design for the TOF TDC electronics.

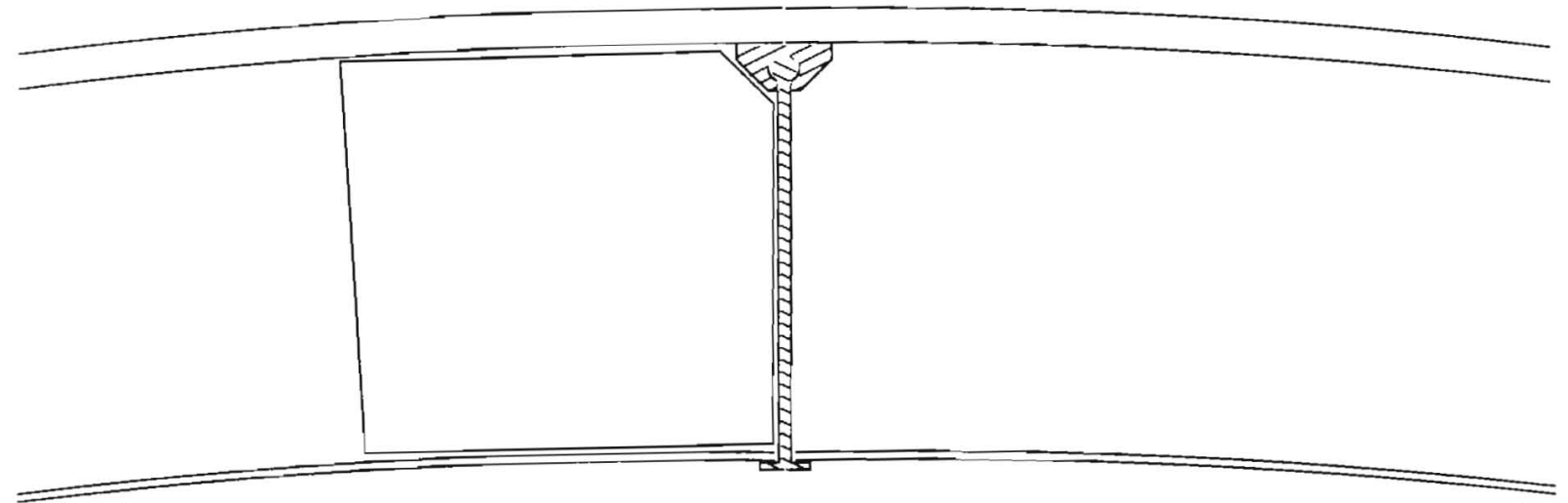

Figure 3.8: Design of the scintillator mounting assembly. 

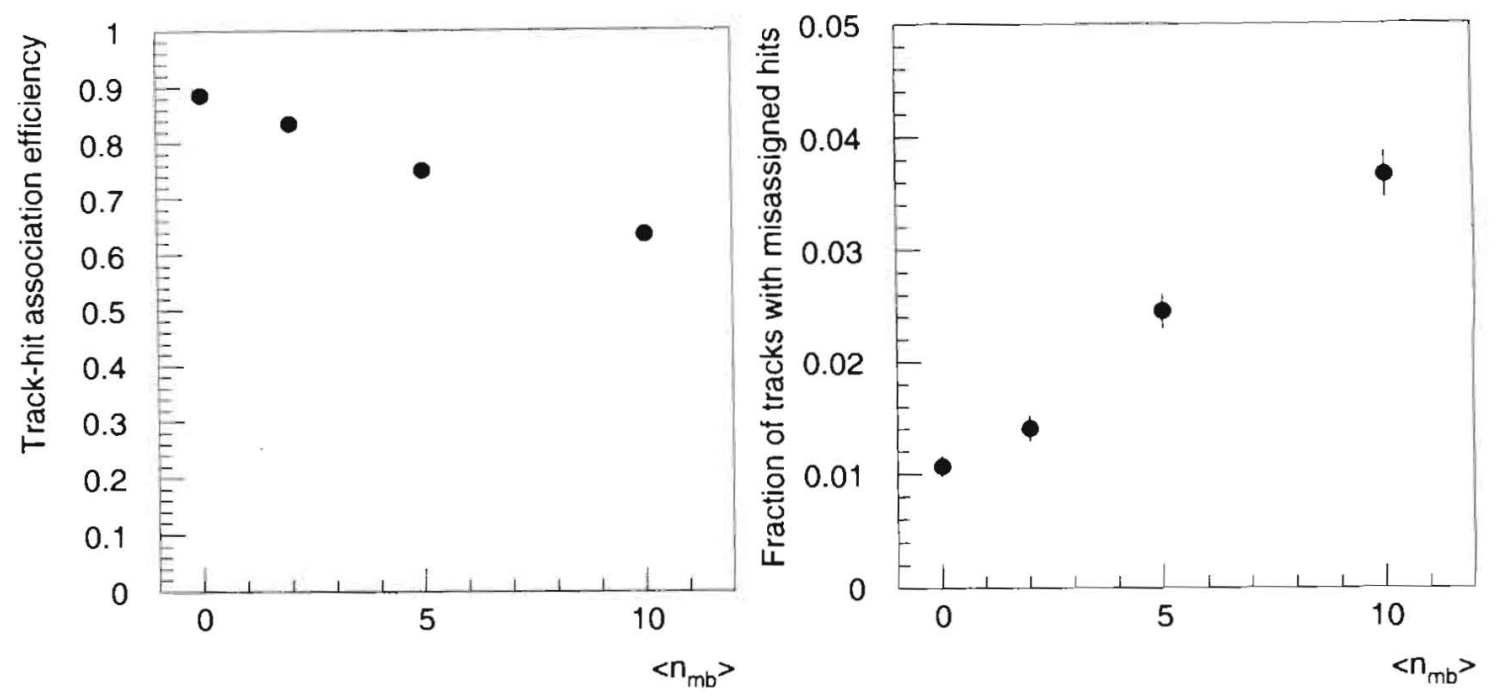

Figure 3.9: Track-hit association efficiency and misassociated fraction of tracks using the " $T_{L}-T_{R}$ " algorithm. These are plotted as functions of the mean number of minimum bias events, $\left\langle n_{\mathrm{mb}}\right\rangle$, present in the event.
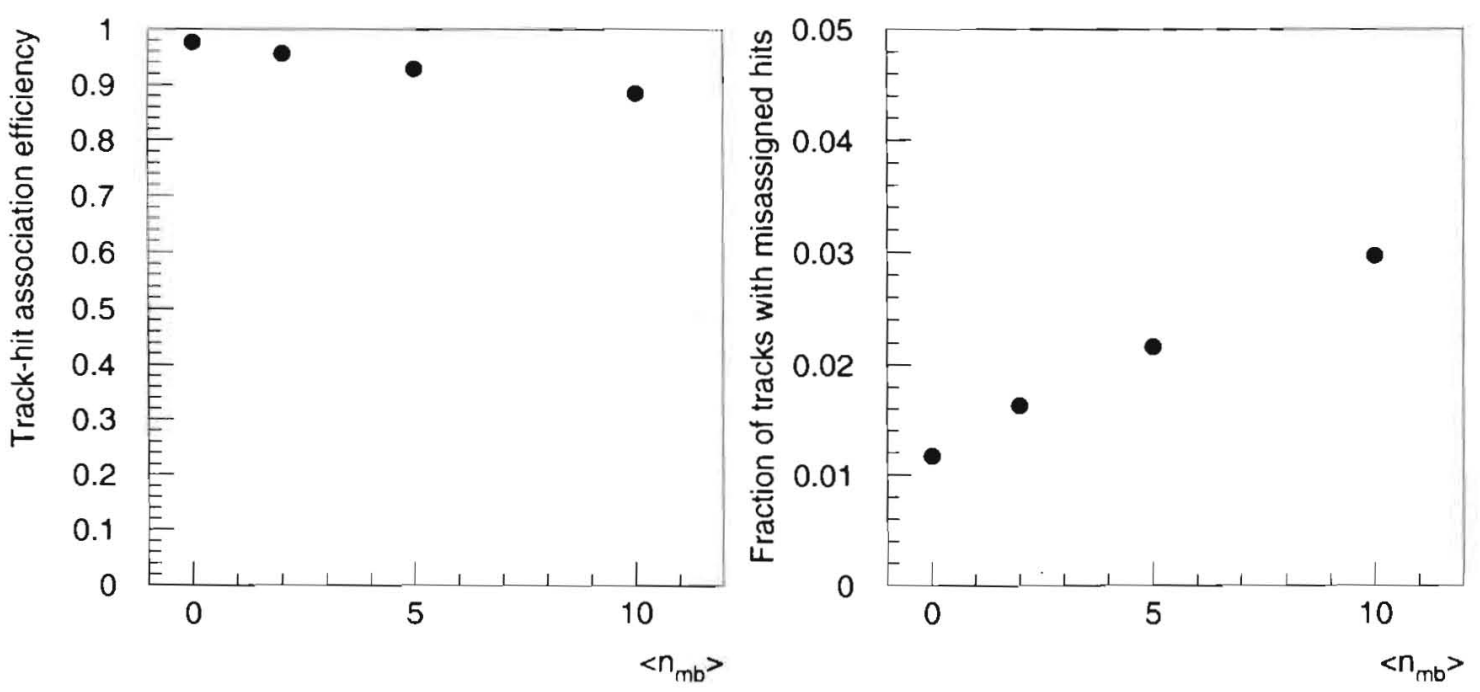

Figure 3.10: Track-hit association efficiency and misassociated fraction of tracks using the "closest track" algorithm. These are plotted as functions of the mean number of minimum bias events, $\left\langle n_{\mathrm{mb}}\right\rangle$, present in the event. 

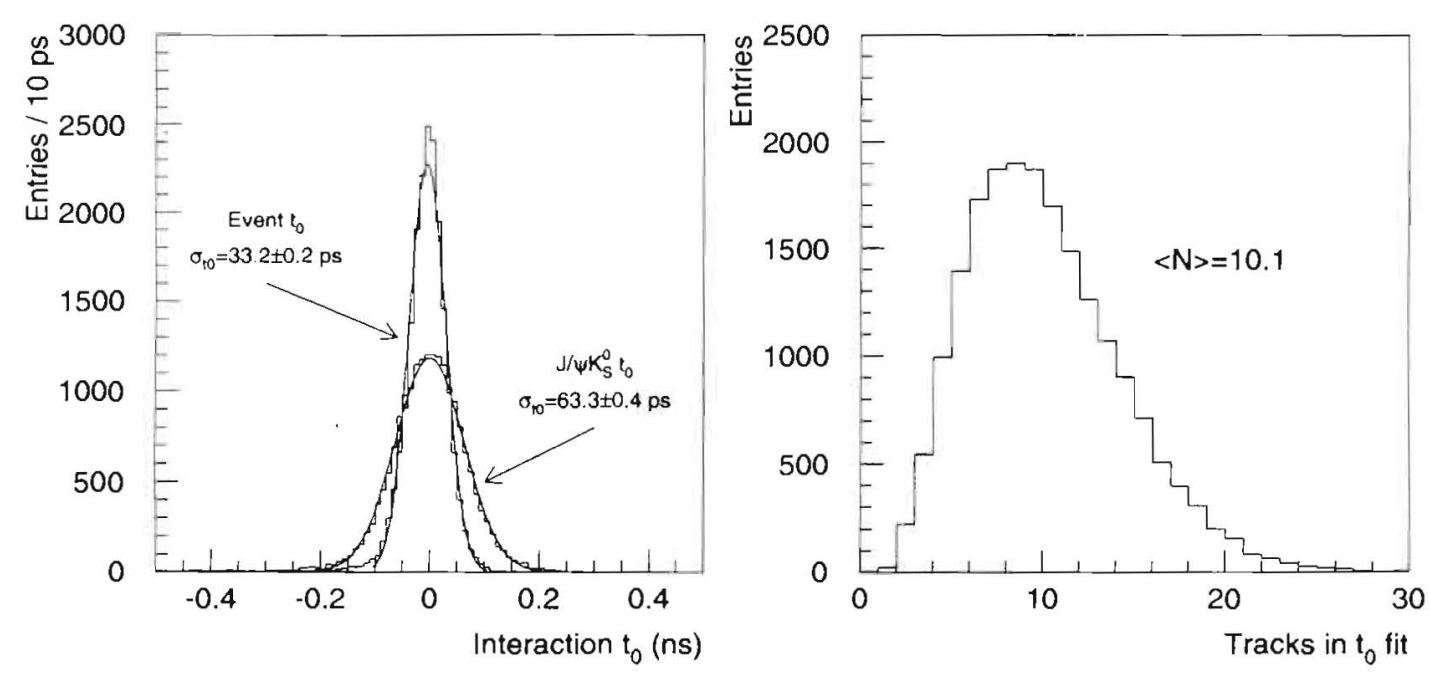

Figure 3.11: Fitted $t_{0}$ distributions for $J / \psi K_{\mathrm{S}}^{0}$ and for the full event. Also shown is the distribution of the number of tracks used in the event $t_{0}$ fit in addition to the tracks from the $B^{0} \rightarrow J / \psi K_{\mathrm{S}}^{0}$ decay.

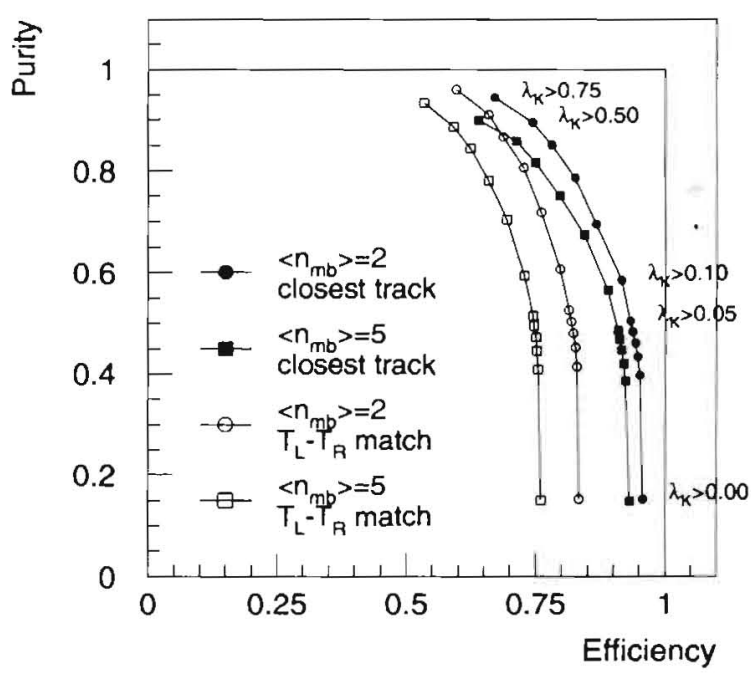

Figure 3.12: A comparison of the efficiency and purity as functions of the normalized likelihood, $\lambda_{K}$, for kaon samples selected using the two methods for associating PMT hits with tracks. It is seen that the algorithm which associates tracks with the closest phototube can provide both improved purity and efficiency compared to the association algorithm which simply requires a match in $z$. The comparison of these algorithms is shown for $\left\langle n_{\mathrm{mb}}\right\rangle=2$ and $\left\langle n_{\mathrm{mb}}\right\rangle=5$. 


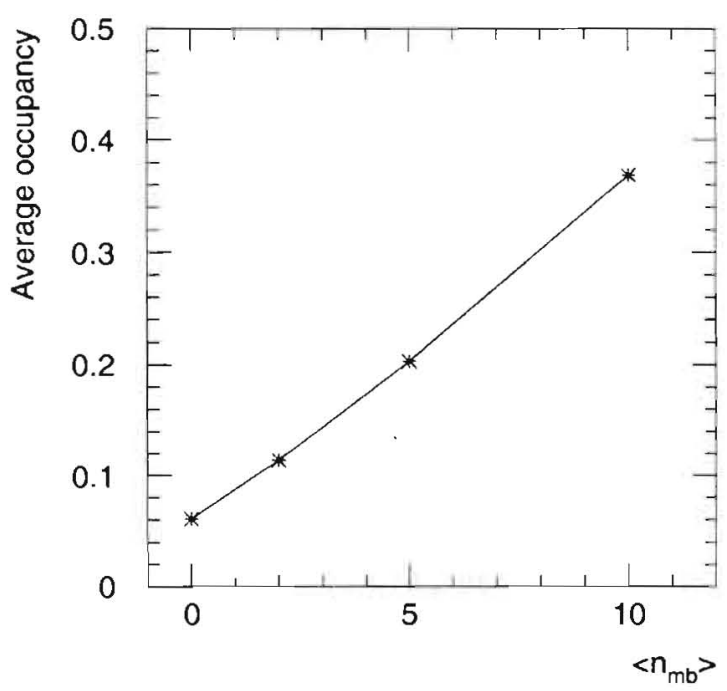

Figure 3.13: Average occupancy expected for the proposed TOF system as a function of the number of minimum bias events.
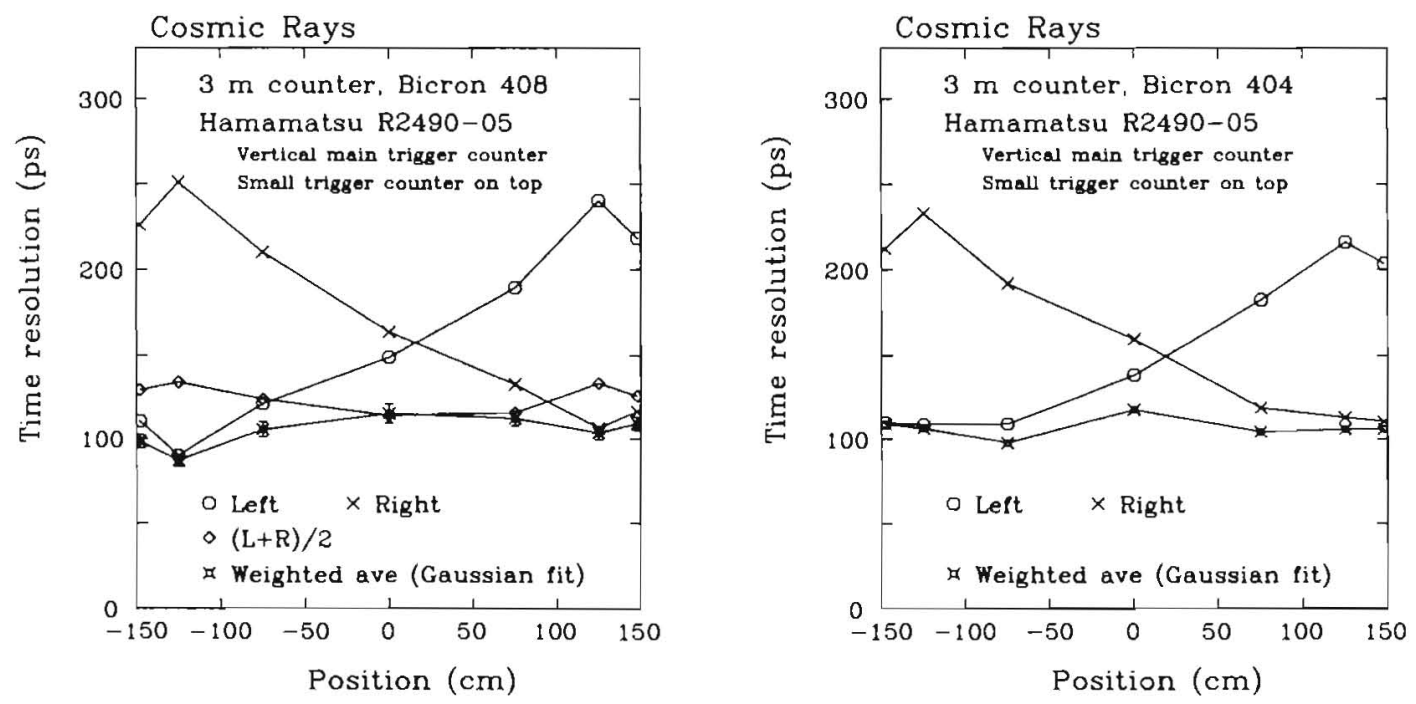

Figure 3.14: Time resolutions measured at several locations along the $3 \mathrm{~m}$ counter. Scintillator on the left is BC408 and on the right is BC404. Phototubes are Hamamatsu R2490. 


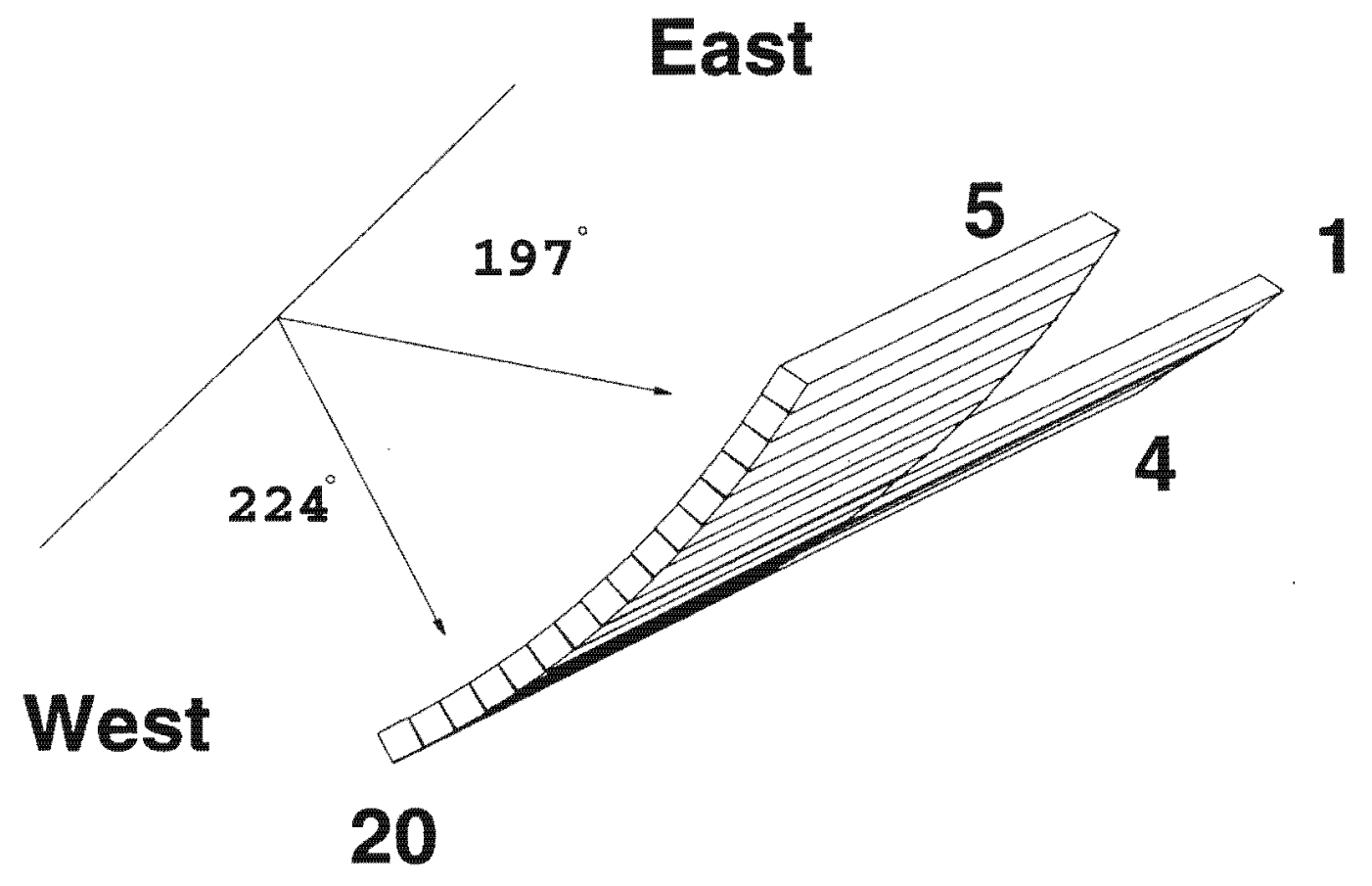

Figure 3.15: TOF counter arrangement (half length bars $130 \mathrm{~cm}$ ) inside the CDF solenoid in the Run Ic test. 


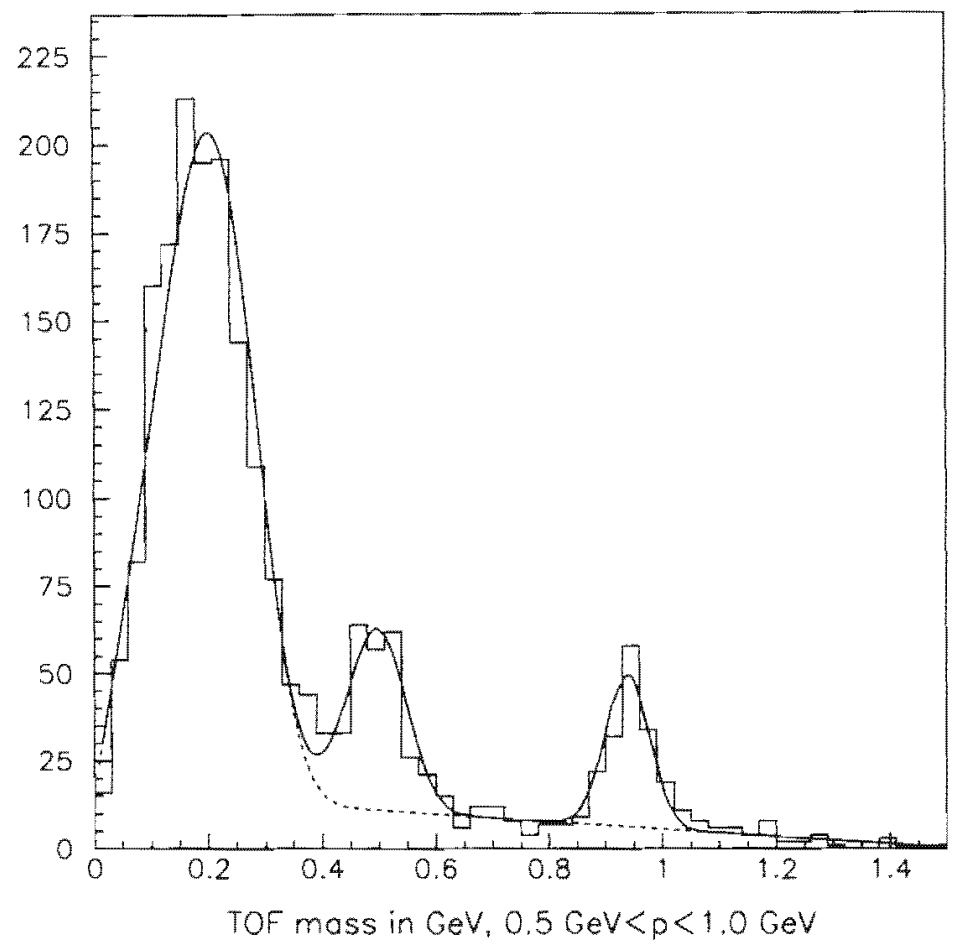

Figure 3.16: The measured TOF mass $\left(m_{f}\right)$ spectrum for tracks with $0.5 \mathrm{GeV} / c$ $<p<1.0 \mathrm{GeV} / c$. The solid line is the result of a fit to the spectrum which includes contributions from pions, kaons, protons, and a linear background. The dashed line shows contribution of only the pion and background terms.
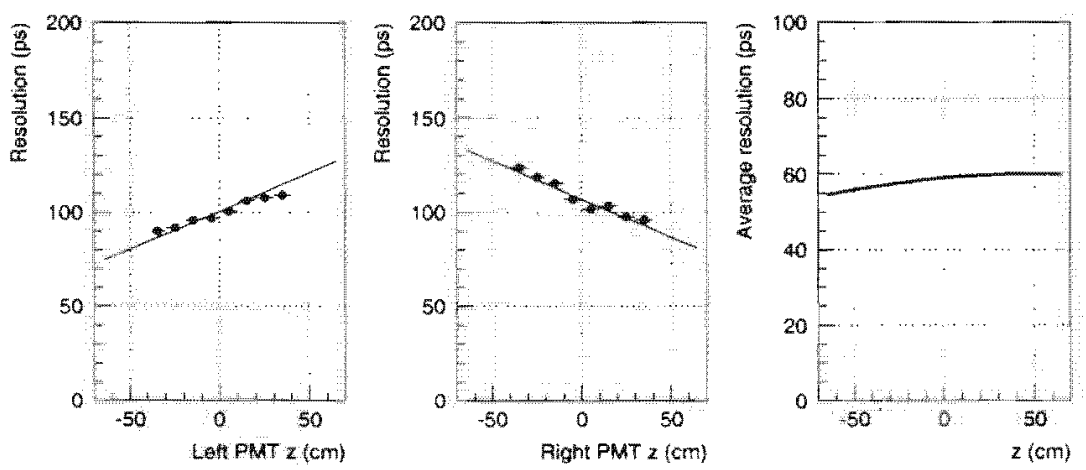

Figure 3.17: Timing resolution from cosmic rays for left and right PMT's as functions of $z$-position. The average resolution, relevant for particle identification, was $\mathcal{O}(60 p s)$ in this study. 


\section{Chapter 4}

\section{Physics with Layer 00}

\subsection{Overview}

The motivations for including Layer 00 (LO0) in the CDF II detector were detailed in Section 2.2. They are:

1. Impact Parameter Resolution. L00 will substantially improve the impact parameter resolution of the CDF II tracking system, particularly for tracks that have low momentum or pass through readout hybrids in SVXII. The estimated resolution improvements are approximately

$$
\sigma=9 \oplus \frac{34}{p_{T}} \longrightarrow 6 \oplus \frac{22}{p_{T}} \mu \mathrm{m}
$$

for tracks that do not pass through SVXII hybrids, and

$$
\sigma=9 \oplus \frac{66}{p_{T}} \longrightarrow 6 \oplus \frac{27}{p_{T}} \mu \mathrm{m}
$$

for tracks that pass through hybrids in each layer of SVXII. (See Table 2.1 for details).

2. Beyond Run II. The L00 silicon sensors are designed to withstand much higher levels of radiation than those in SVXII. If the effectiveness of the innermost layer of SVXII (LO) is reduced by radiation damage, the presence of L00 would mean an almost negligible change in impact parameter resolution allowing continued data taking. L00 can also be regarded as a prototype for radiation-resistant detectors for Run III.

Improved impact parameter resolution will increase the power of CDF II to identify displaced tracks (i.e. tracks that do not come from the primary vertex) and hence tag long-lived particles, i.e. bs and cs. Many important physics channels at the Tevatron Run II will rely on such tagging techniques, with any increase in tagging efficiency translating directly into extra statistical power 
for these analyses. Any improvement in impact parameter resolution will also directly increase the resolution of proper time in mixing measurements.

In fact, the inclusion of L00 will dramatically enhance the identification of decay products from long-lived particles in two ways:

- smaller measurement uncertainties on impact parameters giving easier separation of displaced tracks;

- much better measurement of low $p_{T}$ tracks allowing a lower $p_{T}$ cutoff for tracks to be included in tagging algorithms. This feature is complimented by the ability of the CDF II silicon detectors to do stand-alone tracking; including lower $p_{T}$ tracks that would otherwise have failed to pass through sufficient superlayers to be reconstructed.

The plots in Section 2.2 clearly show the large improvement in resolution at low $p_{T}$. While the importance of this for low $p_{T}$ physics studies is immediately clear, it is also worth noting that high $p_{T}$ physics channels also contain many low $p_{T}$ tracks. For example, as shown in Figure 4.1 , in top production events about half of the observed $b$ descendant particles have $p_{T}<2 \mathrm{GeV}$.

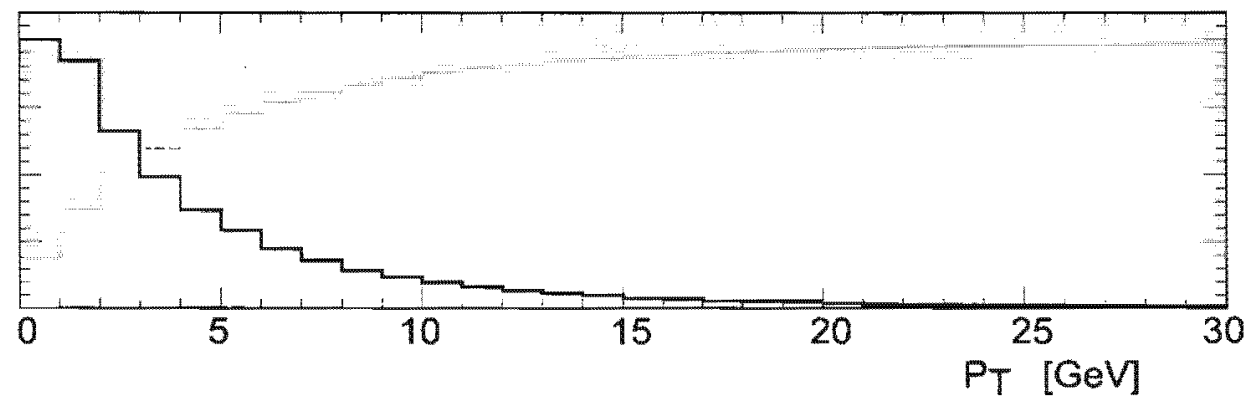

Figure 4.1: $p_{T}$ distribution for charged tracks from $b$ and subsequent $c$ decays in $t \vec{t}$ production events passing trigger and filter requirements. The blue curve shows the integrated distribution.

\subsection{Physics Studies}

Increases in tagging efficiencies for $b$ and $c$ quarks will enhance the signal-to-noise in many physics channels. Increasing the $b$ efficiency benefits all top and bottom physics studies, while both $b$ and $c$ tagging efficiencies can be important in exotic particle searches (e.g. Higgs or technipions decaying to $b \bar{b}$, scalar top decaying to charm plus neutralino, etc.). The improvement in proper time resolution is very 
important for CP violation and mixing measurements (e.g. $B_{s}$ mixing described in Section 5.4).

To quantify the effect of adding L00 to the CDF II tracking system on the type of physics topics mentioned, two analyses have been performed (more are still in progress):

- In high $p_{T} b$-jets in top events, the double $b$-tag efficiency ${ }^{2}$ with L00 included was found to be up to $32 \%$ higher relative to that without L00. If the inner SVXII layer (LO) were to cease functioning, the tagging efficiency would be essentially unaffected with L00 installed but would drop by $15 \%$ without L00. These results are applicable to other high $p_{T}$ processes including many exotic particle searches.

- In low $p_{T}$ b-jets produced in QCD processes, the double b-tag efficiency was found to be up to $289 \%$ higher with L00 than without (i.e. almost four times the efficiency).

These analyses are described in detail in the next three sections. It must be noted that the results obtained are conservative, since the tagging algorithms used were the same as those in Run I. Work is ongoing to develop new algorithms to take advantage of the unique capabilities that L00 and the whole CDF II tracking system will offer.

\subsection{Analysis Tools}

In order to quantify the contribution of L00 to the CDF II physics program, we have written a PYTHIA-based generator program, a parametric detector simulation and the necessary analysis tools. These allow us to generate any desired physics process, smear tracks according to chosen detector resolutions to create $3 \mathrm{D}$ track parameters with properly corrected covariance matrices, and perform physics analyses with the output. As a benchmark, we have used the standard CDF $b$-tagging algorithm "SECVTX" (used for the Run I top analyses) with output ntuples used for various additional tests. To include the effects of primary vertex and alignment uncertainty (as described at the beginning of Section 2.2), the decay length calculated by SECVTX was smeared by $10 \mu \mathrm{m}$ before tagging.

To crosscheck our analysis, we ran our package using SVX' parameters to simulate the $b$-tagging performance of CDF during Run $I$ and compared the results with those from the real Run I data. The single $b$-tag efficiency obtained with the simulation was $49 \%$ compared to $45 \%$ in data. It is expected that the simulation efficiency should be slightly higher since we have not attempted to

\footnotetext{
'Single tag efficiency is defined as the fraction of $b$-jets which are correctly tagged; double tag efficiency is the fraction of events with two b-jets in which both are correctly tagged.
} 
include detector or pattern recognition inconsistencies. Therefore, although we cannot rely on the simulation to give the absolute performance of a detector to a high degree of accuracy, we feel confident in using it to compare the relative performance of different possible configurations.

\section{4 b Tagging in Top Events}

To study the effect of L00 on high $p_{T}$ physics analysis, we simulated $t \bar{t}$ production at $2 \mathrm{TeV}$. The general results are also applicable to any similar physics signatures, including exotic particle decays to $b \vec{b}$ (e.g. Higgs). Using the SECVTX tagging algorithm with standard kinematic cuts, we find that the addition of L00 to SVXII leads to a significant enhancement of single and double $b$-tagging. For $b$-jets in the SVXII layer 0 hybrid regions, the addition of L00 increases the $b$-tag efficiency by $25 \%$. In the low mass regions of SVXII, there is an enhancement in the $b$-tag efficiency of $7 \%$. Overall, the SVXII detector with L00 is found to have a $9 \%$ higher single tag efficiency and a $20 \%$ higher double tag efficiency than without L00, (assuming a $30 \mathrm{~cm}$ luminous region).

Moreover, the use of the SECVTX algorithm with standard kinematic cuts does not give a complete picture of the potential improvements we can expect with L00. Since 250 to $750 \mathrm{MeV}$ tracks with L00 will be as well resolved as $500 \mathrm{MeV}$ to $2 \mathrm{GeV}$ tracks without L00, L00 should enable a reduction in the values of track $p_{T}$ cuts. We therefore also ran the SECVTX algorithm with L00 parameters and "equivalent" $p_{T}$ thresholds, i.e. $p_{T}$ cuts lowered to accept tracks with as good a resolution as those at the higher cuts without L00. We then find that the addition of L00 leads to a $32 \%$ increase in the double tag efficiency.

Finally, one can ask how L00 improves things after SVXII Layer 0 has died. If this occurs with a functioning L00, then there is negligible change in tagging efficiencies. Without L00, there is an $8 \%$ reduction in single tagging and $15 \%$ reduction in double tagging. Thus the relative improvement in efficiency with L00 in this case is $18 \%$ for single tags and $42 \%$ for double tags with standard $p_{T}$ cuts, and $24 \%$ for single tags and $64 \%$ for double tags with equivalent $p_{T}$ cuts.

All of the results of our simulations are summarized in Table 4.1. Note that the results presented are for overall efficiencies, including acceptance factors. For example, the SVX' single tag efficiency of $25 \%$ consists of a $b$ tag efficiency of $49 \%$ multiplied by a geometrical acceptance factor of 0.5 , since only half of the $b$-jets produced pass through the detector.

\subsubsection{Factors Not Included in the Simulation}

As already mentioned, these results do not take into account all the nuances of real tracking. Pattern recognition errors and failures will diminish the tagging rates shown in Table 4.1. Secondary tracks produced in interactions between 


\begin{tabular}{|c||c|c||c|c|}
\hline \multicolumn{1}{|c||}{ Detector } & \multicolumn{2}{c|}{ Single Tag Efficiency } & \multicolumn{2}{c|}{ Double Tag Efficiency } \\
\cline { 2 - 5 } & Absolute & /SVXII & Absolute & /SVXII \\
\hline \hline SVX & $25.0 \pm 0.6 \%$ & 0.55 & $8.0 \pm 0.5 \%$ & 0.38 \\
\hline \hline SVXII & $45.1 \pm 0.7 \%$ & 1.00 & $21.0 \pm 0.8 \%$ & 1.00 \\
\hline SVXII (L0 Dead) & $41.7 \pm 0.7 \%$ & 0.93 & $17.8 \pm 0.8 \%$ & 0.85 \\
\hline \hline L00 (Standard $p_{T}$ cuts) & $49.3 \pm 0.7 \%$ & 1.09 & $25.2 \pm 0.8 \%$ & 1.20 \\
\hline L00 & $\mathbf{5 1 . 8} \pm 0.7 \%$ & $\mathbf{1 . 1 5}$ & $\mathbf{2 7 . 8 \pm 0 . 8 \%}$ & $\mathbf{1 . 3 2}$ \\
\hline L00 (L0 Dead) & $51.8 \pm 0.7 \%$ & 1.15 & $27.8 \pm 0.8 \%$ & 1.32 \\
\hline
\end{tabular}

Table 4.1: B Tag Efficiency Comparisons. Quoted efficiencies include detector acceptance and $10 \mu \mathrm{m}$ decay length smearing. The second and fourth columns show the efficiencies relative to those for the baseline CDF II tracking system. L00 figures are for "Equivalent" $p_{T}$ cuts unless otherwise specified.

particles and material in the detector will also reduce the efficiencies. It is not planned to include these effects in the package described, (although they will naturally be part of the full CDF II tracking simulation currently under development), but it is possible to consider these effects qualitatively.

An additional track hit with high position resolution, as afforded by L0O, is likely to reduce the number of fake tracks and allow an increase in track-finding efficiency. On the other side of the coin, it can be argued that LOO adds material to the system and hence increases the secondary track problem. However, the material added by L00 is minimal $\left(0.6 \% \mathrm{X}_{0}\right.$ over most of the coverage) and it is possible that L00 may prove to be valuable in untangling the effects of secondaries generated at larger radii.

The tracking system as a whole will be more robust with L00 than without. Any unexpected or unconsidered effect leading to a reduction in tagging efficiency should therefore affect the L00 estimates less than those with SVXII alone.

One concern is the possibility that many tracks would have overlapping hits in L00, particularly in dense jets. In our simulations, the fraction of tracks $\left(p_{T}>\right.$ $100 \mathrm{MeV}$ ) having hits within 2 strips of another track in L00 was found to be $23 \%$, assuming a radius of $1.7 \mathrm{~cm}$ and a $50 \mu \mathrm{m}$ readout pitch. This compares with $20 \%$ in SVXII layer 0 (radius $=2.44 \mathrm{~cm}$, pitch $=60 \mu \mathrm{m}$ ). It should be noted that these estimates are conservative, since the $Z$ segmentation was not taken into account.

\subsubsection{Tagging as a Function of $b$ quark $p_{T}$}

Fig.4.2 shows the $b$-tagging efficiencies as a function of the $p_{T}$ of the $b$ quark. Comparing the black and blue entries, we see that when using the standard track $p_{T}$ cuts, L00 gives a relatively constant absolute increase in tagging efficiency at 


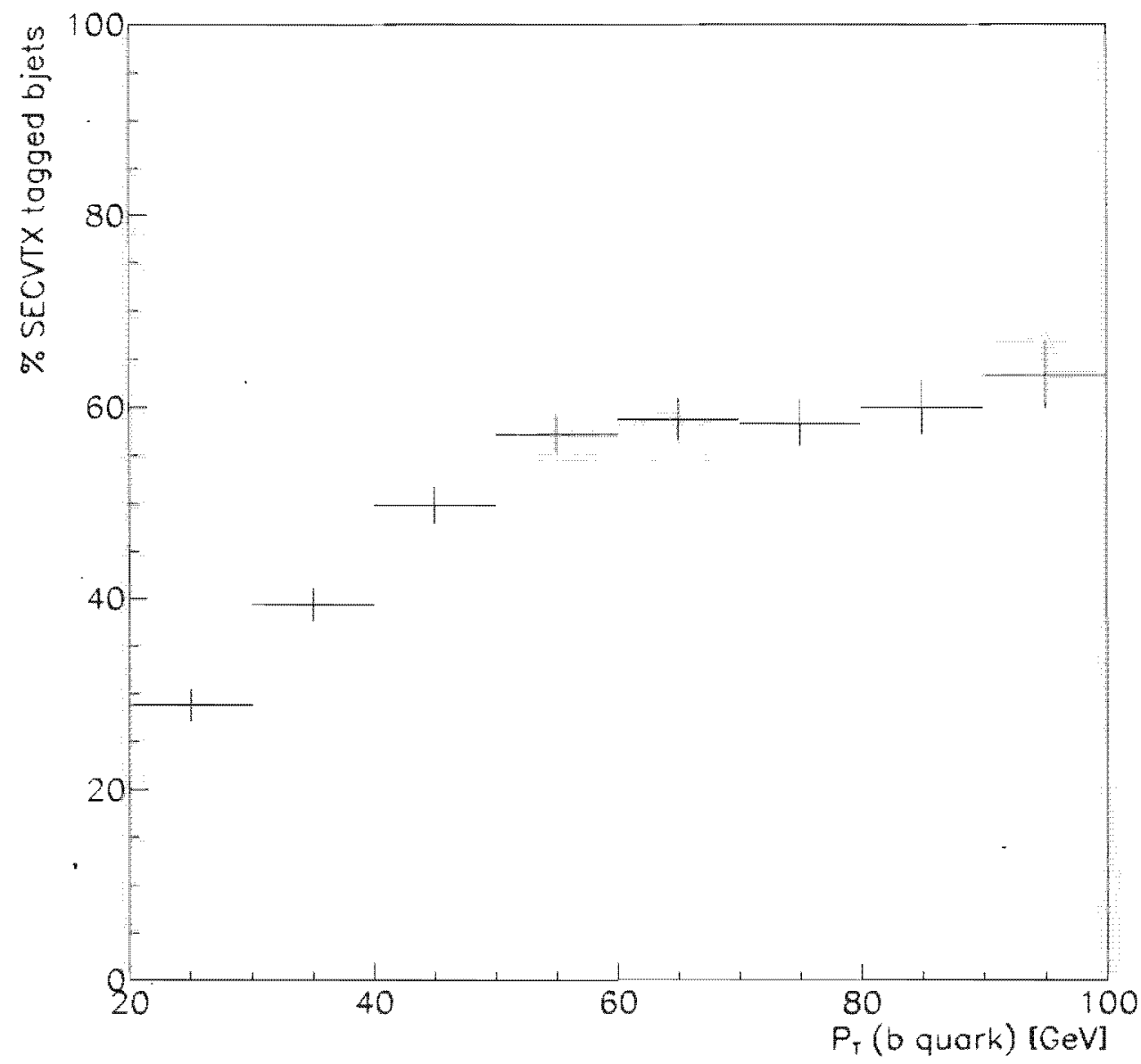

Figure 4.2: $b$-tagging efficiencies as a function of the $b$ quark $p_{T}$. Errors shown are statistical and only plotted once since the same events were used to generate each of the curves. Black: SVXII only; Red: SVXII (L0 Dead); Blue: SVXII+L00; Green: SVXII+LO0 using "Equivalent" $p_{T}$ cuts

all $p_{T}$. The relative increase is unsurprisingly larger for low $b$ quark $p_{T} \mathrm{~s}$ when the lower "equivalent" track $p_{T}$ cuts are used.

\subsection{Displaced Tracks in Low $p_{T} b$ Decays}

To study the impact of LOO on low $p_{T}$ physics topics, we generated and analyzed $b \bar{b}$ events. $b$-jets in these events were then selected by requiring at least one displaced track with $p_{T}>2 \mathrm{GeV}$. Figure 4.3 shows a comparison of the number of significantly displaced tracks observed in these $b$-jets using SVXII with and without LO0.

It is seen that L00 adds more than 0.5 displaced tracks per $b$ decay on average. 


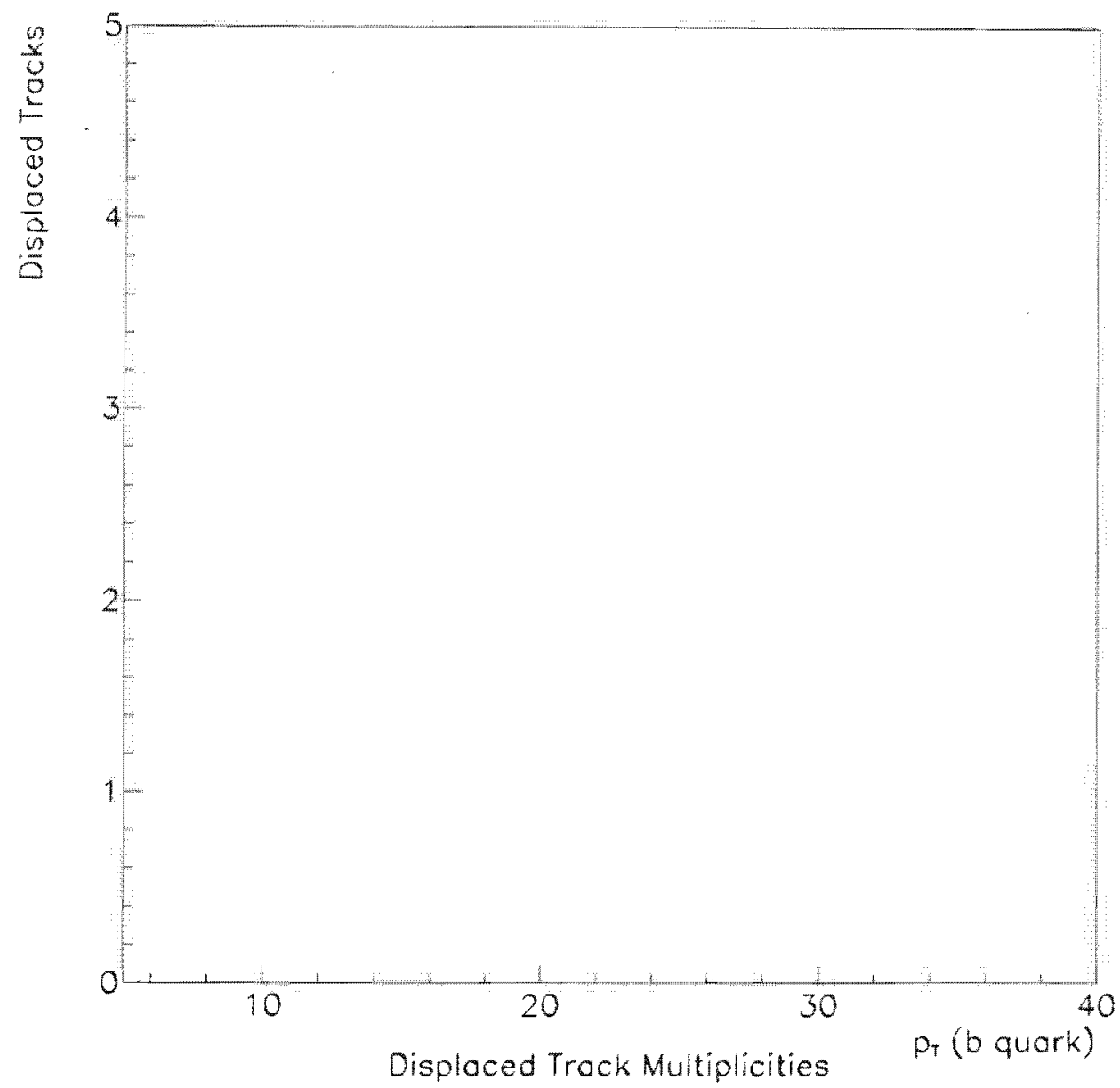

Figure 4.3: Number of displaced tracks in low $p_{T} b$ decays. Red: SVXII; Blue: SVXII +LO0

This can lead to a very significant enhancement in physics capabilities, as is clear when considering that the statistical background for three displaced tracks is so much smaller than that for two. Thus we expect a major improvement in signal to noise, particularly for low $p_{T} b$-jets, giving us the possibility of both new physics channels and greater statistical power in existing ones.

To estimate $b$-tagging efficiencies in this low $p_{T}$ jet sample we increased the maximum allowed angle between the jet and track to one unit in $\eta-\phi$ space (the standard SECVTX algorithm cuts at 0.4). With "equivalent" $p_{T}$ cuts, the addition of L00 results in a $98 \%$ relative increase in the single tag efficiency and a $289 \%$ increase in the double tag efficiency. Even with standard $p_{T}$ cuts the double tag efficiency shows a $55 \%$ increase. This clearly demonstrates the utility of LOO and its power to increase the tracking and analysis capabilities of the CDF II detector. 


\section{Chapter 5}

\section{Physics with Time of Flight}

\subsection{Physics Motivation for TOF}

\subsubsection{Overview}

The proposed TOF system will broadly enhance the physics capabilities of the CDF-II detector. Here we highlight a few of the main physics topics. The TOF system will make a dramatic improvement in the ability to study the physics associated with the $B_{s}$ meson, in particular, $B_{s}$ mixing. This measurement, in conjunction with a measurement of $\sin 2 \beta$, may provide the first test of closure of the unitarity triangle. The TOF system could more than double the $B_{s}$ flavor tagging effectiveness and consequently same-side kaon tagging for $B_{s}^{0}$ may be our most powerful $B$ flavor tag. Furthermore, in the Standard Model, $C P$ asymmetries induced by mixing in $B_{s}^{0}$ decays to $C P$ eigenstates (e.g. $B_{s}^{0} / \bar{B}_{s}^{0} \rightarrow J / \psi \phi$ ) are expected to be extremely small. A measurement of a significant asymmetry would be indisputable evidence that the Standard Model is incomplete. The TOF system also extends the sensitivity to any long-lived charged massive particles. For example, in gauge mediated SUSY breaking models, the stau could be the next-to-lightest SUSY particle and its decay into the gravitino (the lightest SUSY particle) is suppressed. In such models, all SUSY particles cascade down into a long-lived stau. Present CDF-II simulations demonstrate the TOF system will extend the $4 \sigma$ discovery reach of the detector from $\sim 110 \mathrm{GeV} / c^{2}$ out to a stau mass of $\sim 160 \mathrm{GeV} / c^{2}$.

In the following sections we introduce the nomenclature associated with the unitary triangle, we give a brief description of the TOF simulation and then follow with a discussion of flavor tagging. We report first on flavor tagging results from the decay mode $B^{0} \rightarrow J / \psi K_{S}^{0}$ because it has been studied in the CDF RunI data, allowing a comparison of Monte Carlo distributions with data. There are detailed discussions of opposite-side and same-side tagging of $B^{0} \rightarrow J / \psi K_{S}^{0}$, followed by same-side tagging for $B_{s}$. We present the expected improvements to $B_{s}$ mixing and the sensitivity to $C P$ violation in the decay mode $B_{s} \rightarrow J / \psi \phi$ 
from the TOF system. We then outline details of the search for the long-lived supersymmetric tau. Finally, we end with a list of other uses for TOF and a brief summary.

\subsubsection{The Unitary Triangle}

The weak decays of $B$ hadrons are of great interest, because they probe five of the nine elements in the three-generation Cabibbo-Kobayashi-Maskawa matrix. The unitarity of this matrix leads to nine unitarity relationships, one of which is of particular interest:

$$
V_{\mathrm{ud}} V_{\mathrm{td}}^{*}+V_{\mathrm{us}} V_{\mathrm{ts}}^{*}+V_{\mathrm{ub}} V_{\mathrm{tb}}^{*}=0
$$

In the complex-plane, this sum of three complex numbers can be drawn as a triangle, and this triangle is commonly referred to as the Unitary Triangle. The weak decays of $B$ hadrons can be used to measure the magnitudes of the three sides of the Unitary Triangle, and $C P$ asymmetries in $B$ meson decays can be used to measure the three angles. The goal of $B$ physics in the next decade will be to measure both the sides and angles of this triangle to see if they give a consistent picture.

We can use several approximations to reexpress eq. 5.1 in a more convenient form:

$$
\begin{gathered}
V_{\mathrm{ud}} \approx V_{\mathrm{tb}}^{*} \approx 1 \\
V_{\mathrm{ts}}^{*} \approx-V_{\mathrm{cb}}
\end{gathered}
$$

$V_{\text {us }}$ is $\lambda=\sin \theta_{\mathrm{C}}$. Equation 5.1 then becomes

$$
1+\frac{V_{\mathrm{td}}^{*}}{\lambda V_{\mathrm{ts}}^{*}}-\frac{V_{\mathrm{ub}}}{\lambda V_{\mathrm{cb}}}=0,
$$

which is shown as the sum of three vectors in the complex plane in Figure 1.

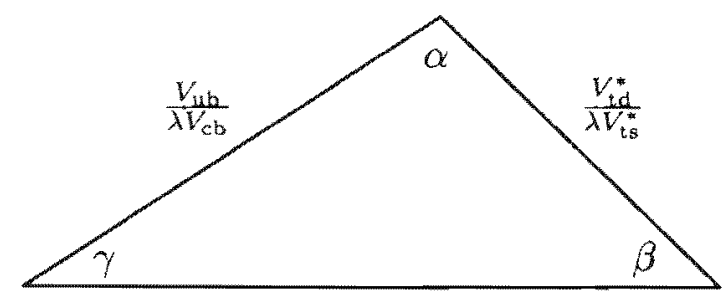

1

Figure 5.1: The Unitarity Triangle with the sides renormalized in a convenient way. 
The Cabibbo angle is already measured to a precision of $1 \%: \lambda=0.2196 \pm$ 0.0023 [28]. The ratio $\left|V_{\mathrm{ub}} / V_{\mathrm{cb}}\right|=0.08 \pm 0.02$ [28], determined from a combination of results from CLEO and LEP, will be measured more precisely by adding data from the asymmetric $B$ factories and CLEO. The angle $\beta$ will be measured both by experiments on the $\Upsilon(4 S)$ and at hadron colliders. The first determination will probably be from measuring $\sin 2 \beta$ from the asymmetry in $B^{0} / \bar{B}^{0} \rightarrow J / \psi K_{\mathrm{S}}^{0}$. The ratio $\left|V_{\mathrm{td}}^{*} / V_{\mathrm{ts}}^{*}\right|$ will probably first be determined by measuring the frequency $\Delta m_{s}$ of $B_{s}^{0} \leftrightarrow \bar{B}_{s}^{0}$ oscillations, and comparing it to the already well measured frequency $\Delta m_{d}$ of $B_{d}^{0} \leftrightarrow \bar{B}_{d}^{0}$ oscillations:

$$
\frac{\left|V_{\mathrm{td}}^{*}\right|}{\left|V_{\mathrm{ts}}^{*}\right|}=(1.15 \pm 0.05) \cdot \sqrt{\frac{\Delta m_{d} \cdot m\left(B_{s}^{0}\right)}{\Delta m_{s} \cdot m\left(B^{0}\right)}}
$$

The first test of unitarity will be a combination of measurements of $\sin 2 \beta$ and $\left|V_{\mathrm{td}}^{*} / V_{\mathrm{ts}}^{*}\right|$. The CDF collaboration is in a unique position in that it can do both. Since $B_{s}^{0}$ mesons are not produced on the $\Upsilon(4 S)$, this physics will be the domain of hadron colliders for some time to come. ${ }^{1}$.

\subsection{3 $B$ Reconstruction and Flavor Tagging}

Measuring flavor oscillations and $C P$ asymmetries requires:

1. reconstructing the $B$ meson in the appropriate final state, e.g., $B^{0} / \bar{B}^{0} \rightarrow$ $J / \psi K_{\mathrm{S}}^{0}$ for $\sin 2 \beta$, or $B_{s}^{0} \rightarrow D_{s}^{-} \pi^{+}$, followed by $D_{s}^{-} \rightarrow \phi \pi^{-}$, with $\phi \rightarrow$ $K^{+} K^{-}$for $\Delta m_{s}$;

2. measuring the proper-time of the $B$ meson decay;

3. determining the flavor of the $B$ meson when it is produced (and in the measurement of flavor oscillations, comparing it to the flavor when it decays). We refer to this determination as "b-flavor tagging." The flavor of the $B$ meson is determined by whether it contains a $b$ quark or $\vec{b}$ antiquark: $B^{0}(\bar{b} d)$ and $B^{+}(\bar{b} u)$ have the same flavor, but $B^{+}$and $B^{-}$have opposite flavor.

The Time-of-flight detector (TOF) is useful for identifying pions, kaons, and protons. In the context of $B$ physics, particle identification is useful for both reconstructing $B$ decays and for $b$ flavor tagging. The primary motivation for proposing this upgrade is $b$ flavor tagging. The figure of merit for a $b$ flavor tagging method is its "total tagging effectiveness", $\epsilon D^{2}$. Here the efficiency $\epsilon$ is how often you can apply the tag given you have a $B$ decay (such as $J / \psi K_{\mathrm{S}}^{0}$ ) that you want to tag: $0<\epsilon<1$. D is known as the "dilution" and is related to the probability $\mathcal{P}$ that the flavor tag is correct: $D=2 \mathcal{P}-1$. A value of $D=1$ (or

\footnotetext{
'In the next years, depending on the performance of the SLC collider, the SLD collaboration could achieve a sensitivity of $\Delta m_{s}=15 \hbar \mathrm{ps}^{-1}$.
} 
"dilution" $=100 \%$, corresponding to $\mathcal{P}=1$ ) describes a perfect flavor tag that is always correct; a value $D=0$ (or "dilution" $=0 \%$, corresponding to $\mathcal{P}=0.5$ ) describes a random tag.

The statistical error on the determination of a $C P$ asymmetry $\mathcal{A}$ determined with $N$ total candidates is approximately

$$
\delta \mathcal{A} \approx \frac{1}{\sqrt{\epsilon D^{2} N}}
$$

An additional flavor tagging method that increases $\epsilon D^{2}$ by a factor of two leads to a decrease in the statistical error on $\mathcal{A}$ by a factor of $\sqrt{2}$ (and the same decrease on the error of, for example, the $\mathrm{CP}$ violation parameter $\sin 2 \beta$ ). A value of $\epsilon D^{2}$ of $1 \%$ or more is respectable.

Using Run I data, CDF has investigated several $b$ flavor tagging methods:

1. the soft-lepton flavor tag or SLT;

2. the jet-charge flavor tag or JCT;

3. the same-side flavor tag or SST.

The SLT and JCT are "opposite-side" flavor tagging methods, that is, they identify the flavor of the other $B$ hadron in the event to infer the flavor of the $B$ hadron of interest. The SLT is based on identifying the semileptonic decay of the $B$ hadron. The charge of the lepton identifies the $b$ flavor of the $B$ hadron: $b \rightarrow \ell^{-}$, but $\bar{b} \rightarrow \ell^{+}$. The JCT is based on the momentum weighted sum of charges of tracks in the jet formed by the $b$ quark. This charge is on average negative for a hadron containing $a b$ quark and positive for a hadron containing a $\bar{b}$ antiquark.

The SST is based on the charge correlation of tracks produced in association with the hadronization of a $\bar{b}$ quark into a $B$ meson. In particular, a $\pi^{+}\left(\pi^{-}\right)$ is expected to be produced in association with the formation of a $B^{0}\left(\vec{B}^{0}\right)$. Figure 5.2 illustrates the view we have for the fragmentation of a $\bar{b}$ quark into a $B^{+}$or $B^{0}$ meson. To form a $B^{+}$, a $\bar{b}$ quark combines with a $u$ quark from a $u \bar{u}$ pair, pulled from the vacuum, which leaves a $\bar{u}$ quark available for the formation of the charged hadrons $\pi^{-}, K^{-}$or $\bar{p}$. For $B^{+}$, all charged hadrons formed in this way have the same charge, which is opposite that of the primary $\bar{b}$ quark charge. However, for a $B^{0}$ meson, a positive charge correlation occurs only when a $\pi^{+}$is formed. Same-side tagging methods rely on identifying fragmentation tracks produced in association with a $B$ meson and using their charge to tag the production flavor.

The performance of these $b$ flavor tagging methods has been quantified by measuring $B^{0}$ flavor oscillations, which also requires knowledge of the flavor of the neutral $B$ meson at production. For the SLT we measure $\epsilon D^{2}=(1.07 \pm$ $0.13) \%[29]$; for the JCT we measure $\epsilon D^{2}=(0.78 \pm 0.15) \%[29]$; and for the 


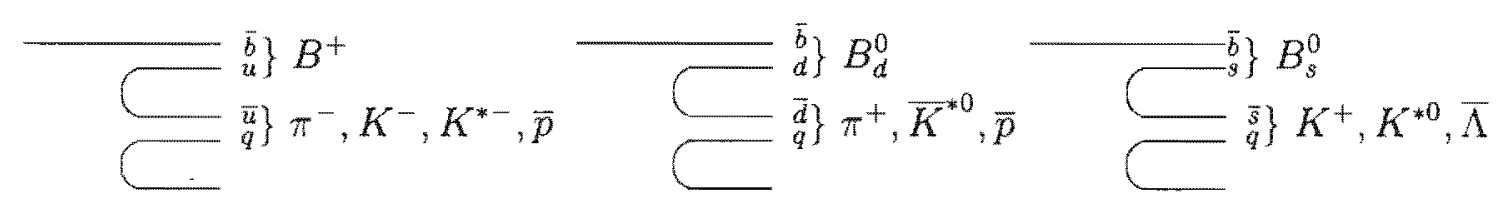

Figure 5.2: A schematic picture of $B^{+}, B^{0}$, and $B_{s}^{0}$ meson formation in $\bar{b}$ quark fragmentation. $A B^{+}$is produced in association with positively charged hadrons only, while a $B^{0}$ is produced along with positive pions, negative kaons (via $\bar{K}^{* 0} \rightarrow$ $K^{-} \pi^{+}$) and antiprotons. A $K^{+}$is produced in association with a $B_{s}^{0}$.

SST we measure $\epsilon D^{2}=\left(2.4_{-0.8}^{+0.9}\right) \%[6]$ using a sample of partially reconstructed semileptonic $B^{0}$ decays. Based on a combination of Monte Carlo studies and measurements from our data, we determine $\epsilon D^{2}=(1.8 \pm 0.3) \%$ [2] in our $B^{0} / \bar{B}^{0} \rightarrow J / \psi K_{\mathrm{S}}^{0}$ data sample for the SST.

These Monte Carlo studies used the PYTHIA [30] Monte Carlo, with parameters tuned [31] to reproduce the charged multiplicities and $p_{T}$ distributions observed in the data. We have found [32] that PYTHIA explains well the SST dilutions measured in our data, and reproduces several distributions associated with the selection of tracks used for same-side tagging. Fig. 5.3 shows an example. In the following sections, we rely on PYTHIA, using the same parameters, for our projections for RUN II flavor tagging efficiencies.

The TOF can be used to enhance both opposite-side and same-side $b$ flavor tagging. The decays of $\bar{B}$ hadrons containing $b$ quarks produce more $K^{-}$than $K^{+}$on average. Therefore identifying a charged kaon from $B$ decay can be used as an opposite-side $b$ flavor tag. This is the basis of one of the two flavor tags that will be used by the $B$ factories (the other is the lepton-tag). The TOF can be used for same-side tagging as well. In the case of $B_{s}^{0}$ production, the same mechanism described above for $B^{0}$ produces a $K^{+}\left(K^{-}\right)$in association with the formation of a $B_{s}^{0}\left(\bar{B}_{s}^{0}\right) . \quad B_{s}^{0}$ physics is especially important, because it will be unique to the Tevatron in Run II. In addition to using kaon identification to enhance SST for $B_{s}^{0} / \bar{B}_{s}^{0}$, we can also use it to enhance SST for $B^{0} / \bar{B}^{0}$. In this case, however, the kaons produced in association with $B^{0} / \bar{B}^{0}$ have the opposite charge- $b$ flavor correlation expected for pions (see figure 5.4). Therefore we will either use the TOF to identify kaons (and protons too, by the way) and reject them, or to switch their charge correlation for the flavor tag. 


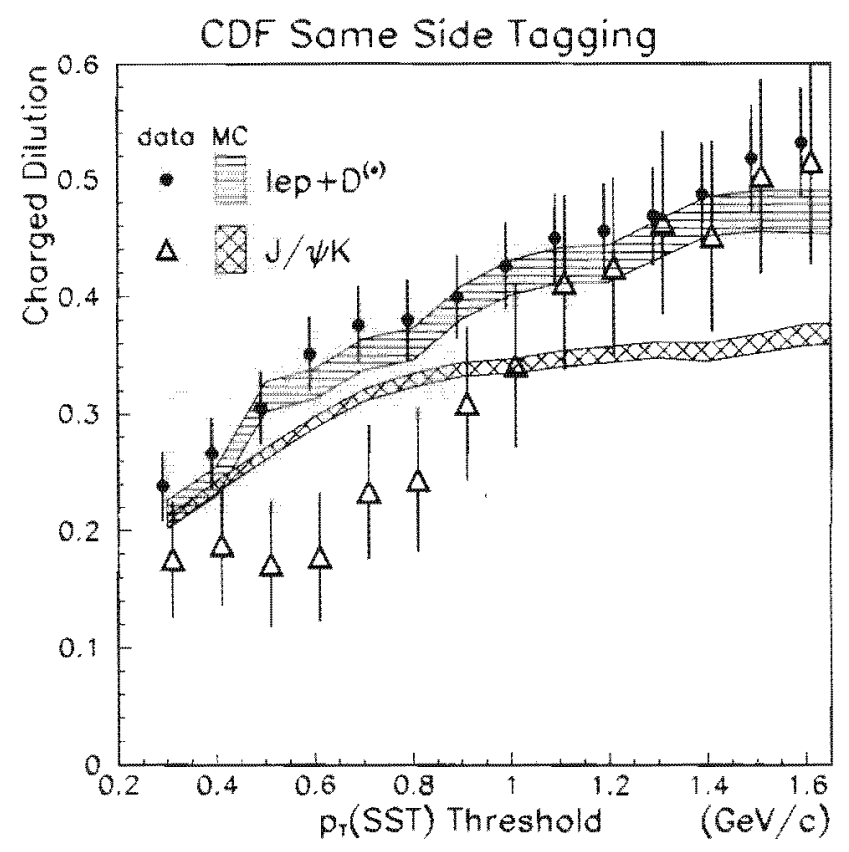

Figure 5.3: Comparison of data with predictions from PYTHIA [30], for the tagging dilution as a function of $\operatorname{tag} p_{t}$ cut for $B^{+}$data. The data points are correlated with each other.

\subsection{Time-of-Flight Simulation}

We have written a simulation of TOF that can be used for fast parametric studies. A detailed description of the simulation can be found in reference [27]. The helical trajectories of charged particles in the magnetic field are determined. Particles with $p_{T}<330 \mathrm{MeV} / c$ curve so much in the magnetic field that they never hit the TOF. We approximate the COT tracking efficiency as $100 \%$ for charged particles with $p_{T}>400 \mathrm{MeV} / c$. Particles with $330<p_{T}<400 \mathrm{MeV} / c$ are not used in physics analyses, but their affect on TOF counter occupancy is taken into account. In addition, the simulation includes:

- The time-of-flight resolution, including the effects of fluctuations in photon statistics in the scintillator. The average resolution for a single particle is $100 \mathrm{ps}$.

- The decay in flight of kaons and pions before they enter the TOF.

- Occupancy effects, including shadowing of one track by another (we have not proposed multiple hit electronics), the effect of additional inelastic collisions (minimum bias events), and photon conversions in the scintillator and outer can of the COT. 


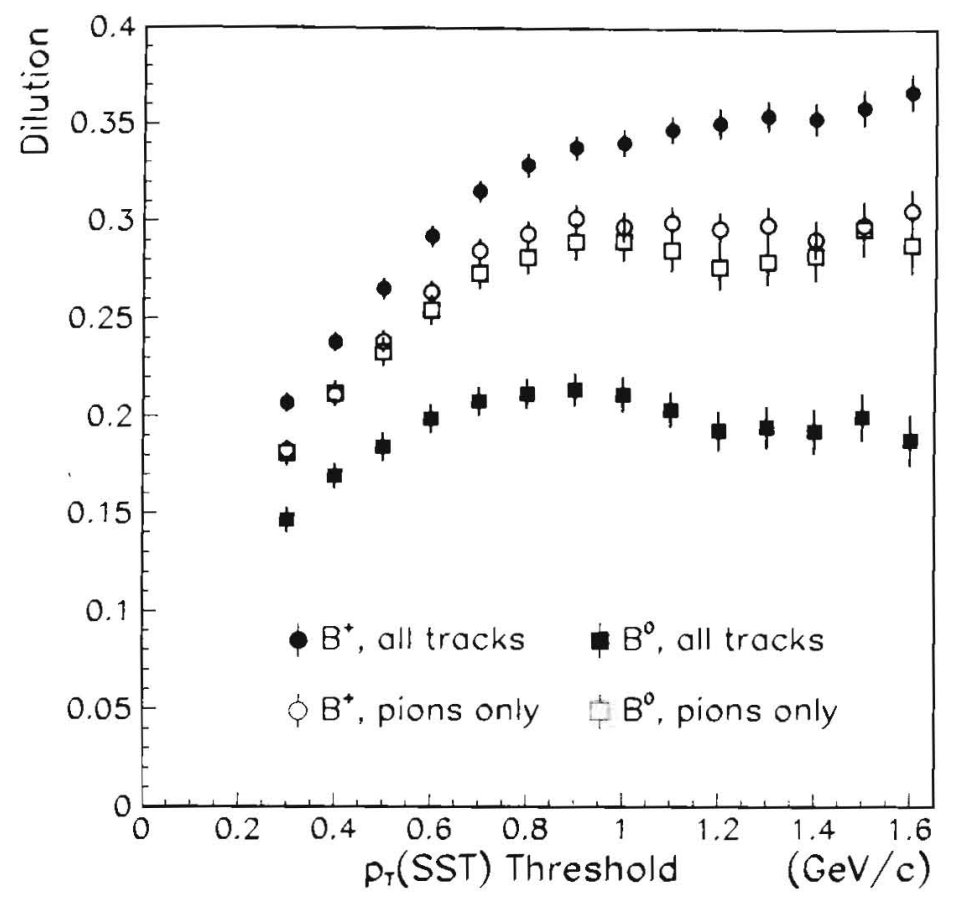

Figure 5:4: Dilution as a function of the $\operatorname{tag} P_{T}$ cut from Monte Carlo simulations from the $J / \psi K$ modes of $B^{0}$ 's (solid squares) and $B^{+}$'s (solid circle). When the tagging is restricted to prompt pions only, the neutral dilution becomes the open squares, and the charged the open circles.

- The resolution on the time $t_{0}$ measured with TOF of the $p \bar{p}$ interaction.

In opposite-side kaon tagging, we are searching for kaons coming from the decay of $B$ hadrons. We must separate these kaons from kaons coming from the underlying event and additional inelastic collisions. We can achieve this separation efficiently by exploiting the relatively long lifetime of $B$ and $D$ hadrons. The kaons from $B$ hadron decay should have significant impact parameters with respect to the primary vertex. The simulation uses a parameterization of the impact parameter resolution of tracks, both for the currently proposed SVXII geometry, and for SVXII plus an additional layer of silicon (Layer 00) with $r \varphi$ readout only, located inside SVXII.

\subsection{Results on $b$ Flavor Tagging using the TOF}

\subsubsection{Flavor Tagging for $B^{0} / \bar{B}^{0} \rightarrow J / \psi K_{\mathrm{S}}^{0}$}

To study opposite-side and same-side flavor tagging for the measurement of $\sin 2 \beta$, we have generated a sample of $b \bar{b}$-events using the PYTHIA Monte 

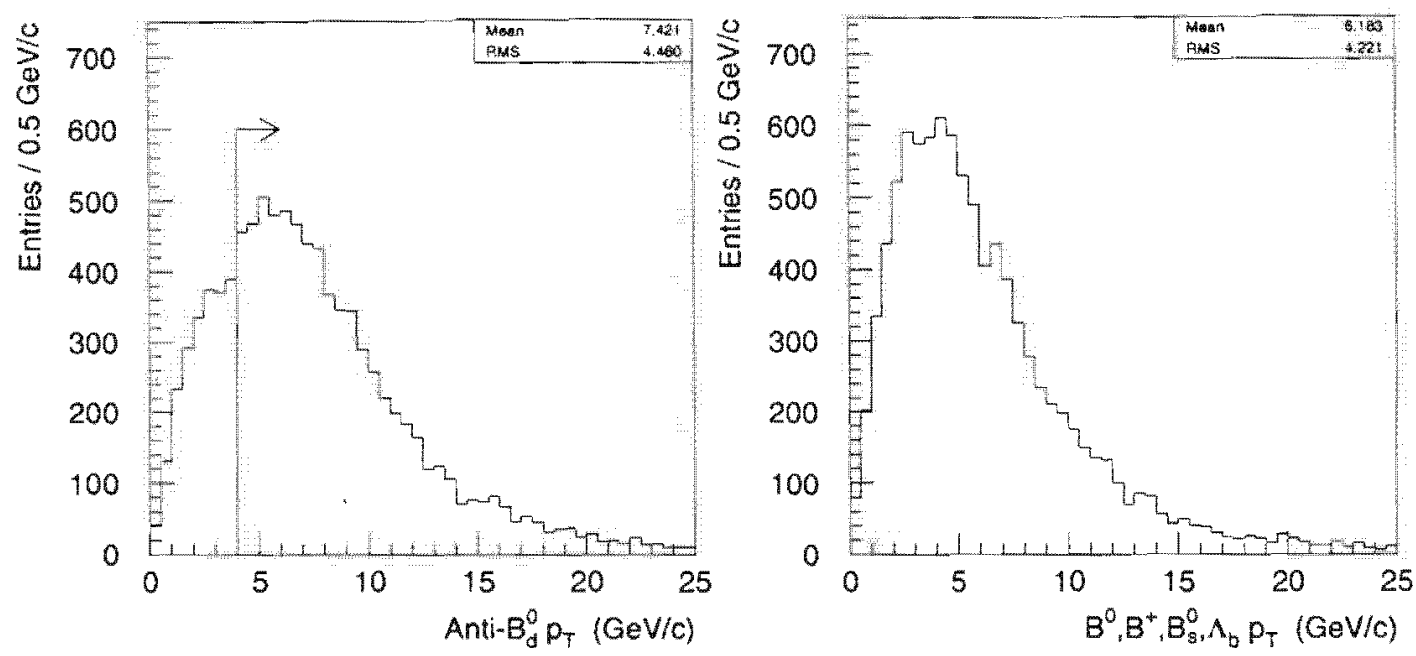

Figure 5.5: Transverse momentum distributions for the $\bar{B}^{0}$ that decayed to $J / \psi K_{\mathrm{S}}^{0}$ (left) and the opposite-side $B$ hadrons (right).

Carlo [30]. The $b \bar{b}$ events were generated with the same parameters used in our published studies [6] on same-side tagging, as described in Section 5.1.3. The $B$ hadrons in these events were decayed using $Q Q$ [33] with a modified decay table that forced the decays $\bar{B}^{0} \rightarrow J / \psi K_{\mathrm{S}}^{0}$ and $J / \psi \rightarrow \mu^{+} \mu^{-}$. A sample of 20,000 such events were generated and were used for the studies of both same-side and opposite-side tagging.

To simulate the Run II trigger, we required the two muons from $J / \psi \rightarrow \mu^{+} \mu^{-}$ to be within $|\eta|<1$, and each muon was required to have transverse momentum larger than $1.5 \mathrm{GeV} / c$. We also required that the $K_{\mathrm{S}}^{0}$ have $|\eta|<1$ and $p_{T}>$ $1 \mathrm{GeV} / c$. The resulting $p_{T}$ distributions for the $\bar{B}^{0}$ and for the opposite-side $B$ hadron are shown in Fig. 5.5. Finally, when analyzing these data, we require the $p_{T}$ of the reconstructed $\bar{B}^{0}$ be greater than $4 \mathrm{GeV} / c$ because this is the lowest $P_{T}$ used in Run-I CDF analyses that gives a good signal-to-noise in the mass peak. This reduces the data sample by $25 \%$, and increases the mean $p_{T}$ of the $\bar{B}^{0}$ from $7.3 \mathrm{GeV} / c$ to $8.9 \mathrm{GeV} / c$.

\section{Opposite-Side Tagging in $B^{0} / \bar{B}^{0} \rightarrow J / \psi K_{\mathrm{S}}^{0}$}

The charged kaon multiplicity in flavor tagged $B$ decays has been measured by ARGUS and DELPHI. The multiplicities produced by the $B$ meson decay package QQ agree with the measurements at the $20 \%$ level. Unfortunately, the inclusive branching fraction $\operatorname{Br}\left(B \rightarrow K^{ \pm} X\right)$ has not been measured.

The excess of positive kaons in $B$ decays provides a means to tag the flavor of an opposite side $B$. We construct a flavor tag by selecting tracks identified as kaons that are separated from the $J / \psi K_{\mathrm{S}}^{0}$ and that have impact parameters inconsistent with production at the primary vertex. $A B$ is tagged if there is exactly one kaon selected in this way according to its charge. No attempt is 
made to tag the remaining small fraction of the events (14\%) in which two or more kaons have been selected. The momentum spectrum of opposite-side kaons from $B$ decay is shown in Fig. 5.6. The spectrum is soft and hence a TOF system will tag over one-half of the decays with $>2 \sigma$ separation.

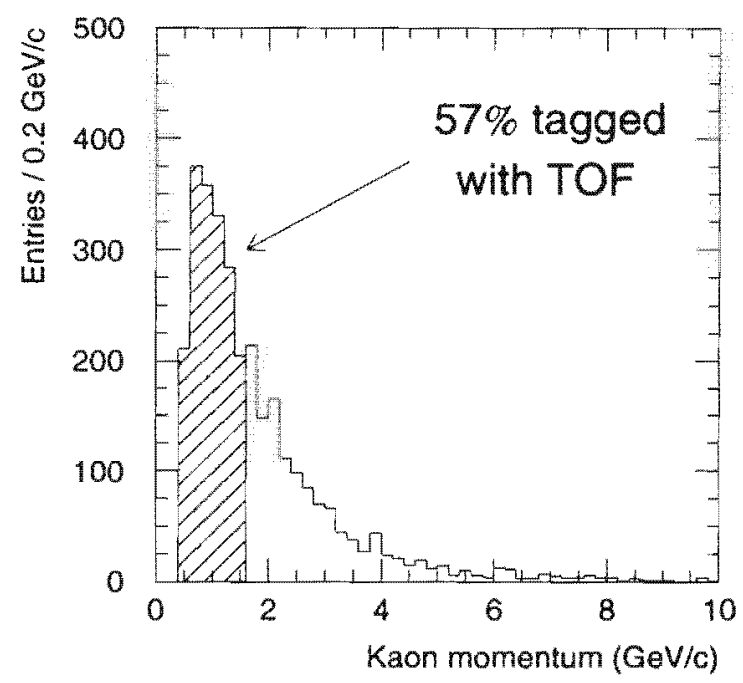

Figure 5.6: Momentum spectrum of kaons from the $B$ decay opposite $\bar{B}^{0} \rightarrow$ $J / \psi K_{\mathrm{S}}^{0}$ from events with $p_{T}\left(\bar{B}^{0}\right)>4 \mathrm{GeV} / c$. Opposite side kaons are required to have $p_{T}>400 \mathrm{MeV} / c$ and $|\eta|<1$. We find $57 \%$ of these kaons have momentum less than $1.6 \mathrm{GeV} / c$ and would be identified using TOF.

The acceptance for an opposite side $B$ to have only one (at least one) kaon with $p_{T}>0.4 \mathrm{GeV} / c$ that hits a $3 \mathrm{~m}$ TOF bar was found to be $0.144 \pm 0.004$ $(0.167 \pm 0.004)$. We did not simulate neutral $B$ meson mixing in the PYTHIA Monte Carlo, but we reverse the charge of the selected kaons if they originated from a $B^{0}$ or $B_{s}^{0}$ with probabilities

$$
\begin{aligned}
\mathcal{P}\left(B_{s}^{0} \rightarrow \overline{B_{s}^{0}}\right) & =\frac{1}{2} \\
\mathcal{P}\left(B^{0} \rightarrow \overline{B^{0}}\right) & =\frac{1}{2}\left(1-\cos \Delta m_{d} t\right)
\end{aligned}
$$

where $m_{d}=0.472 \mathrm{ps}^{-1}[34]$ and $t$ is the decay time of the $B^{0}$, generated with an average lifetime of $1.59 \mathrm{ps}$.

An upper bound on $\epsilon D^{2}$ can be estimated by considering only those kaons that were decay products of the opposite side $B$ hadron. This gives a tagging efficiency of $0.144 \pm 0.004$ with a dilution of $0.67 \pm 0.02$ to give a combined effective tagging efficiency of $\epsilon D^{2}=(6.5 \pm 0.4) \%$. In a real analysis, this will be reduced by the kaon selection efficiency and purity, and by the efficiency with which tracks from the primary vertex are rejected.

The selection of kaons is performed by applying the cut on the likelihood variable $\lambda_{K}>0.25$. The rejection of tracks from the primary vertex was first 


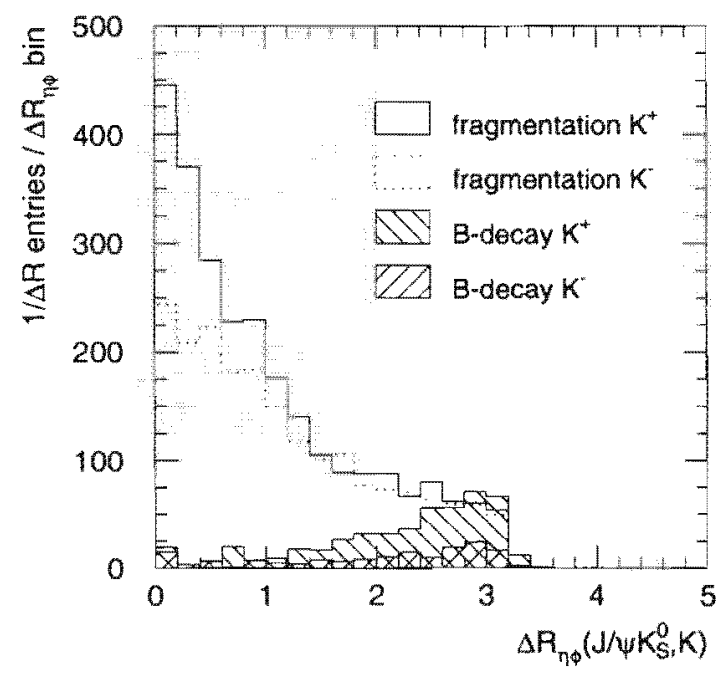

Figure 5.7: Distributions of $\Delta R_{\eta \varphi}$ for kaons from fragmentation, the underlying event, and from the decay of the opposite-side $B$. Entries are weighted by $1 / \Delta R_{\eta \varphi}$

performed by requiring that the kaon candidates be separated in $\eta$ - $\varphi$ space from the $J / \psi K_{S}^{0}$. The $\Delta R_{\eta \varphi}$ distributions of kaons in the Monte Carlo is shown in Fig. 5.7. The kaons from $B$ decays generally populate the region away from the $J / \psi K_{\mathrm{S}}^{0}$ jet, while an excess of "same-side" kaons can be seen around the $J / \psi K_{\mathrm{S}}^{0}$. We select kaons for the tag by requiring $\Delta R_{\eta \varphi}>1$.

To purify this sample further, the transverse and longitudinal impact parameters and their uncertainties for each track were used to construct a probability weight, $w_{D_{0}} z_{0}$, under the hypothesis that the track originated from the primary vertex. Tracks from the primary vertex have weights distributed uniformly between 0 and 1 , while tracks from displaced secondary vertices will generally have weights peaked near zero. The distributions of the weights for these two classes of tracks are shown in Fig. 5.8. We require kaon candidates to have $w_{D_{0} Z_{0}}<0.01$ to be considered for the flavor tag.

Applying this algorithm to select opposite-side kaons gave

$$
\begin{aligned}
\epsilon & =0.100 \pm 0.003 \\
D & =0.44 \pm 0.02 \\
\epsilon D^{2} & =0.019 \pm 0.001
\end{aligned}
$$

for no additional minimum bias events. Figure 5.9 plots the value for the opposite-side tagging $\epsilon D^{2}$ for for $\left\langle n_{\mathrm{mb}}\right\rangle=0,2,5$ and 10 additional minimum bias events.

The tight cut on $w_{D_{0} z_{0}}$ is necessary to obtain a pure sample of tracks from the decays of the opposite-side $B$ hadron. However, the efficiency for tracks from $B$ decays passing this cut would be increased if their impact parameters were measured more precisely. The proposed "Layer 00 " silicon detector would be 


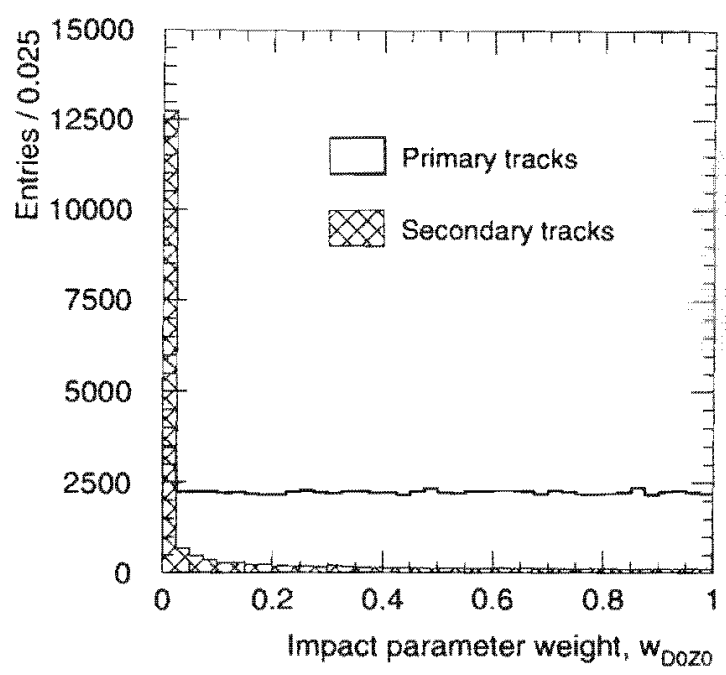

Figure 5.8: Probability weight distributions for tracks produced at the primary vertex (open histogram) and produced in the decay chain of $B$-hadrons (hatched histogram).

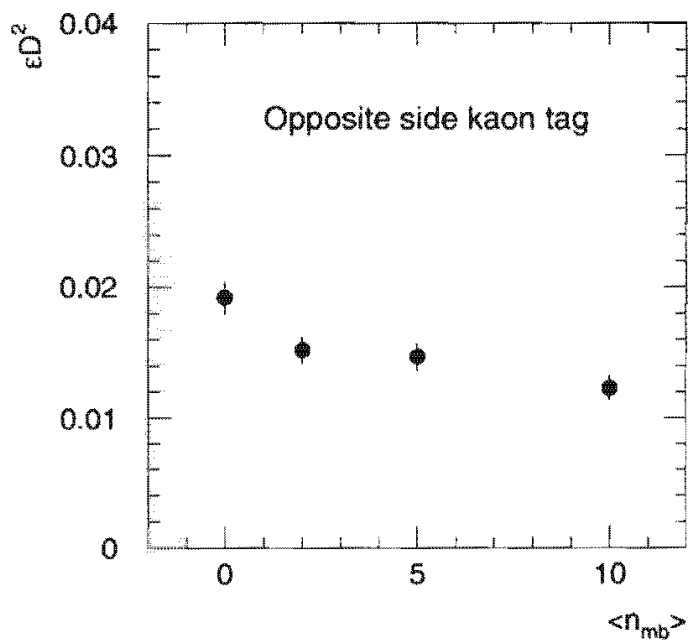

Figure 5.9: $\epsilon D^{2}$ for opposite-side kaon tagging for an average of $0,2,5$ and 10 additional minimum bias events. 
located very close to the beam pipe and would provide $D_{0}$ measurements with a resolution approximately half that of SVXII. We repeated the previous analysis of opposite-side kaon tagging except in this case, the $D_{0}$ resolution including Layer 00 [27] was used. This gave

$$
\begin{aligned}
\epsilon & =0.112 \pm 0.003 \\
D & =0.461 \pm 0.022 \\
\epsilon D^{2} & =0.024 \pm 0.002 \quad \text { (with Layer 00) }
\end{aligned}
$$

for no additional minimum bias events. With $\left\langle n_{\mathrm{mb}}\right\rangle=2$, we obtained $\epsilon D^{2}=$ $0.020 \pm 0.001$.

The need to reject kaon candidates that have impact parameters consistent with the primary vertex is not necessarily an optimal procedure. Instead, it might be better to apply a high efficiency secondary vertex reconstruction algorithm to the opposite-side tracks from which the kaon candidates would be selected. This will studied in the future, when we have a full tracking simulation package. Nevertheless, the simplicity of the analysis presented here indicates that opposite-side tagging should be feasible even without sophisticated vertexing algorithms.

Same-side Tagging in $B^{0} / \bar{B}^{0} \rightarrow J / \psi K_{\mathrm{S}}^{0}$

We use the same-side tagging algorithm used in the CDF measurement of $B^{0} \leftrightarrow \bar{B}^{0}$ mixing [6] and in the CDF measurement of $\sin 2 \beta$ [2]. This considers all charged particles with $p_{T}>400 \mathrm{MeV} / c$ within an $\eta-\varphi$ cone of radius $\Delta R_{\eta \varphi}=0.7$, centered along the direction of the $J / \psi K_{\mathrm{S}}^{0}$ momentum vector. Since we are considering only fully reconstructed $B$ decays, we do not apply a cut on the impact parameters to select tracks from the primary vertex. For each track considered, the quantity $p_{T}^{\text {rel }}$ is calculated, which is defined as the transverse momentum of the track with respect to the combined momentum vector of the $B$ and the track. If no track can be found in an event that satisfies these criteria, the event is not tagged. If at least one such track is found, the production flavor is tagged according to the charge of the track with the minimum $p_{T}^{\text {rel }}$.

We compare the performance of this algorithm to one that makes use of the particle identification capabilities that would be provided by time-of-flight. In this modified same-side tagging algorithm, we consider tracks with $p_{T}>$ $400 \mathrm{MeV} / c$ within the $\eta-\varphi$ cone as before, but also require that they be consistent with being pions when they have TOF hits associated with them. This consistency is made based on the the normalized pion likelihood by requiring $\lambda_{\pi}>0.1$. If the track has no TOF information, it is still considered as a sameside tagging candidate. The resulting flavor tagging efficiency, dilution, and $\epsilon D^{2}$ are shown in Table 5.1 for events with no additional minimum bias events. When we use the minimum $p_{T}^{\text {rel }}$ algorithm and no additional information from TOF, our values of efficiency $(\epsilon=0.582 \pm 0.004)$, dilution $(D=0.155 \pm 0.012)$, and $\epsilon D^{2}$ agree well with the values obtained by CDF and published in [6]. 


\begin{tabular}{|c|c|c|}
\cline { 2 - 3 } \multicolumn{1}{c|}{} & without TOF & with TOF \\
\hline$\epsilon$ & $0.582 \pm 0.004$ & $0.539 \pm 0.004$ \\
$D$ & $0.155 \pm 0.012$ & $0.187 \pm 0.012$ \\
$\epsilon D^{2}$ & $0.014 \pm 0.001$ & $0.019 \pm 0.001$ \\
\hline
\end{tabular}

Table 5.1: Flavor tagging efficiencies, purities and $\epsilon D^{2}$ for same-side tagging of $B^{0}$ using $p_{T}^{\text {rel }}$ with and without TOF used for the identification of associated pions.

The additional information provided by TOF improves the $\epsilon D^{2}$ by over $30 \%$. Figure 5.10 shows how $\epsilon D^{2}$ changes depending on the mean number of minimum bias events present.

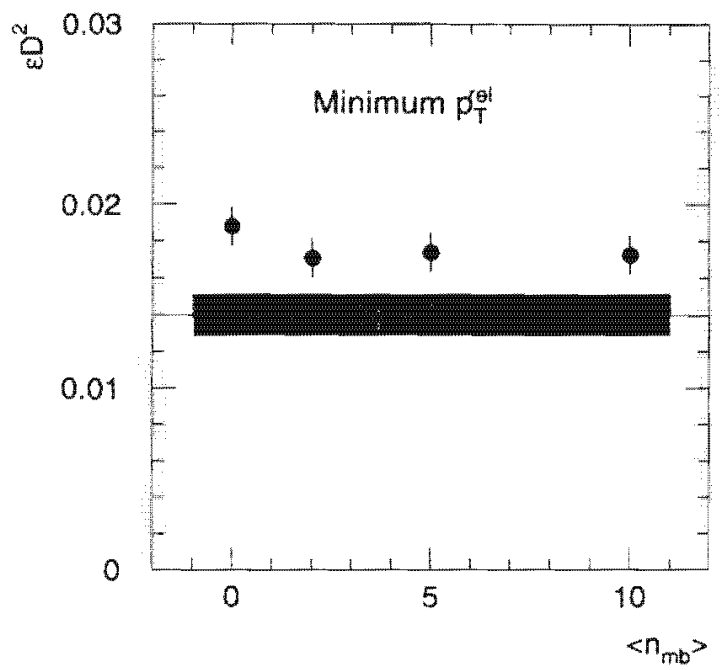

Figure 5.10: $\epsilon D^{2}$ vs $\left\langle n_{\mathrm{mb}}\right\rangle$ for same-side tagging using the Min $p_{T}^{\mathrm{rel}}$ algorithm with TOF (points with error bars) and without particle identification (shaded band).

In this analysis, the degradation of $\epsilon D^{2}$ with increasing $\left\langle n_{\mathrm{mb}}\right\rangle$ is minimal, mainly because the pion identification is only required if a track has associated TOF hits. Thus, the same pion with minimal $p_{T}^{\text {rel }}$ will be chosen as the tag even if its TOF information is lost due to occupancy.

\subsubsection{Same-side kaon tagging for $B_{s}^{0}$}

The same-side tagging principle also applies when a $B_{s}^{0}$ is formed, except that an associated kaon is produced in the fragmentation chain, as shown schematically in Fig. 5.2. The ability to identify kaons in a sample of same-side tag candidates can provide a very powerful method for tagging the production flavor of $B_{s}^{0}$ mesons. 
To study same-side tagging in $B_{s}^{0}$ decays, we generated approximately 7800 $\bar{B}_{s}^{0} \rightarrow J / \psi \phi$ decays using the PYTHIA Monte Carlo. We imposed the same requirements on the decay products as in the $\bar{B}^{0} \rightarrow J / \psi K_{\mathrm{S}}^{0}$ sample, except that we required $p_{T}(\phi)>1 \mathrm{GeV} / c$, instead of $p_{T}\left(K_{S}^{0}\right)>1 \mathrm{GeV} / c$. Again, when analyzing these data, we required the $p_{T}$ of the reconstructed $\bar{B}_{s}^{0}$ be greater than $4 \mathrm{GeV} / c$.

We apply the same-side tagging algorithm described in section 5.3.1 to the $\bar{B}_{s}^{0} \rightarrow J / \psi \phi$ sample, except that in this case we impose a cut on the kaon likelihood, requiring all tag candidates to have $\lambda_{K}>0.25$. The charge of the candidate track selected in this way with the smallest $p_{T}^{\text {rel }}$ is used to identify the production favor. The resulting flavor tagging efficiency, dilution and $\epsilon D^{2}$, for a TOF system and no minimum bias events are

$$
\begin{aligned}
\epsilon & =0.172 \pm 0.004 \\
D & =0.385 \pm 0.025 \\
\epsilon D^{2} & =0.026 \pm 0.002 .
\end{aligned}
$$

Figure 5.11 shows $\epsilon D^{2}$ for same-side kaon tagging for $\left\langle n_{\mathrm{mb}}\right\rangle=0,2,5$, and 10 events added to the $B_{s}$ decay and the nominal underlying event.
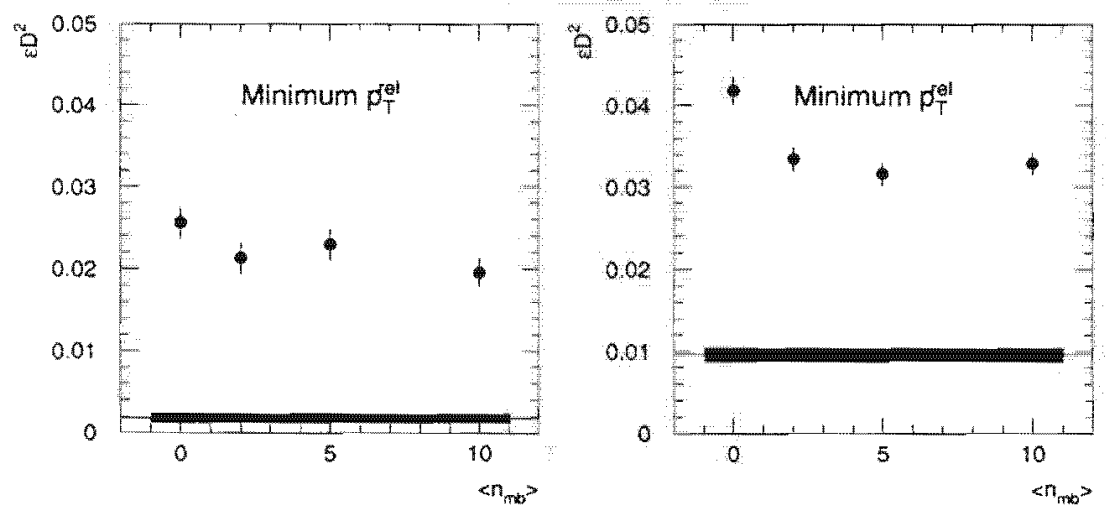

Figure 5.11: The left figure shows $\epsilon D^{2}$ vs $\left\langle n_{\mathrm{mb}}\right\rangle$ for same-side kaon tagging in $B_{s}^{0} \rightarrow J / \psi \phi$ using the Min. $p_{T}^{\text {rel }}$ algorithm. The shaded region shows the value of $\epsilon D^{2}$ obtained with no particle identification. The right figure shows $\epsilon D^{2}$ vs $\left\langle n_{\mathrm{mb}}\right\rangle$ for same-side kaon tagging in $B_{s}^{0} \rightarrow D_{s} \pi^{-}$using the Min. $p_{T}^{\text {rel }}$ algorithm.

These results are strongly dependent on the $p_{T}$ spectrum of the $B_{s}^{0}$ decays being studied. For the previous results we imposed $p_{T}\left(B_{s}^{0}\right)>4 \mathrm{GeV} / c$. If the acceptance is restricted to $p_{T}\left(B_{s}^{0}\right)>8 \mathrm{GeV} / c$, we obtain $\epsilon D^{2}=0.039 \pm 0.004$ for the minimum $p_{T}^{\mathrm{rel}} \mathrm{tag}$. This is particularly interesting since $B_{s}^{0}$ decays tagged with an all-hadronic trigger will have a harder $p_{T}$ distribution.

To investigate this further, we used PYTHIA to generate a Monte Carlo sample of approximately $11000 \overline{B^{0}}{ }_{s} \rightarrow D_{s}^{+} \pi^{-}$decays, where $D_{s}^{+} \rightarrow \pi^{+} \phi$ and $\phi \rightarrow$ $K^{+} K^{-}$. A Level 1 and Level 2 hadronic track trigger was simulated following one 
of the proposed scenarios for Run II [35]. This trigger requires two oppositely charged tracks with $|\eta|<1$, each of which has $p_{T}>2 \mathrm{GeV} / c$. The scalar sum of their $p_{T}$ was required to be greater than $5.5 \mathrm{GeV} / c$, and the azimuthal angle between them was required to be $\delta \varphi<135^{\circ}$. Since the $\pi^{-}$from the $B_{s}^{0}$ decay has the hardest $p_{T}$ spectrum, we consider the two oppositely charged tracks to be either $\pi^{+} \pi^{-}$or $\pi^{-} K^{+}$. The $p_{T}$ spectrum of the accepted $B_{s}^{0}$ decays is shown in Fig. 5.12 and has a mean of about $12 \mathrm{GeV} / c$. Also shown in this Figure is the $p_{T}$ distribution of the kaons from $\phi \rightarrow K^{+} K^{-}$, which indicates that about $45 \%$ of these kaons have $p_{T}<1.7 \mathrm{GeV} / c$. A significant fraction of these kaons will then be distinguishable from pions using time-of-flight for particle identification.
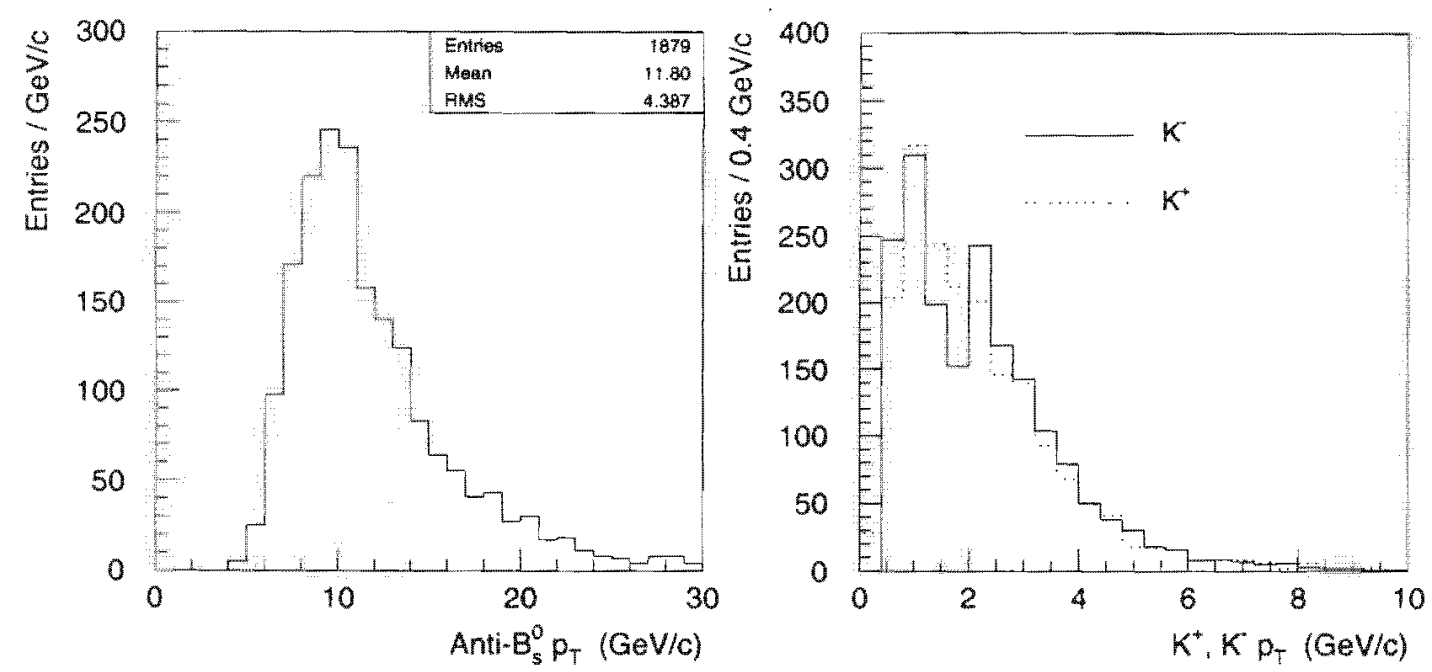

Figure 5.12: $p_{T}$ distribution of $B_{s}^{0} \rightarrow D_{s}^{-} \pi^{+}$decays selected with a hadronic track trigger and of the $K^{ \pm}$from $\phi \rightarrow K^{+} K^{-}$.

Applying the same-side kaon tagging algorithm using $p_{T}^{\text {rel }}$ to this sample gives

$$
\begin{aligned}
\epsilon & =0.170 \pm 0.006 \\
D & =0.496 \pm 0.016 \\
\epsilon D^{2} & =0.042 \pm 0.003 .
\end{aligned}
$$

Same-side tagging using kaons in $B_{s}^{0}$ decays promises to add significantly to our overall $\epsilon D^{2}$; indeed it may turn out to be the most powerful single flavor tagging method.

\section{Summary of $b$ Flavor Tagging with TOF}

Tables 5.2, 5.3, and 5.4 summarize our expected $b$ flavor tagging effectiveness in Run II with and without the TOF. The flavor tagging effectiveness of the sameside tag depends on whether the neutral $B$ meson is a $B^{0}$ or $B_{s}^{0}$, and on $p_{T}(B)$. As a result the total $\epsilon D^{2}$ for $B^{0} / \bar{B}^{0} \rightarrow J / \psi K_{\mathrm{S}}^{0}$ (summarized in Table 5.2), 
$B_{s}^{0} / \bar{B}_{s}^{0} \rightarrow J / \psi \phi$ (summarized in Table 5.3), and $B_{s}^{0} \rightarrow D_{s}^{-} \pi^{+}$(summarized in Table 5.4) are different. The values of $\epsilon D^{2}$ projected for Run II for the soft-lepton tag and the jet-charge tag are taken from the values quoted in the CDF-II Technical Design Report. The values of $\epsilon D^{2}$ for same-side tagging and for opposite-side kaon tagging are the values reported in this proposal. For opposite-side kaon tagging, we use the value of $\epsilon D^{2}$ that is obtained when both TOF and Layer 00 are used. The potential variation in the effectiveness of the opposite-side flavor tags with the $p_{T}$ of the $B$ that triggers the event has been neglected, since this variation is expected to be much smaller than in the case of the same-side tag. Studies of applying multiple $b$ flavor tags to our Run I $B^{0} / \bar{B}^{0} \rightarrow J / \psi K_{\mathrm{S}}^{0}$ data sample indicate that adding up the $\epsilon D^{2}$ values of the individual tags is a good approximation of the total tagging efficiency.

\begin{tabular}{|l|l|l|}
\hline \multicolumn{3}{|c|}{$B^{0} / \bar{B}^{0} \rightarrow J / \psi K_{\mathrm{S}}^{0}$} \\
\hline & without TOF & with TOF \\
\hline \hline Same-Side (pion) Tag & 1.4 & 1.9 \\
Soft Lepton Tag $(\mu)$ & 1.0 & 1.0 \\
Soft Lepton Tag $(e)$ & 0.7 & 0.7 \\
Jet Charge & 3.0 & 3.0 \\
Opposite-Side Kaon Tag & - & 2.4 \\
\hline Total & $6.1 \%$ & $9.0 \%$ \\
\hline
\end{tabular}

Table 5.2: Projected values for $\epsilon D^{2}$ for Run II without and with TOF. These flavor tagging efficiencies are for $B^{0} / \bar{B}^{0} \rightarrow J / \psi K_{\mathrm{S}}^{0}$.

\begin{tabular}{|l|l|l|}
\hline \multicolumn{3}{|c|}{$B_{s}^{0} / \bar{B}_{s}^{0} \rightarrow J / \bar{\phi}$} \\
\hline & without TOF & with TOF \\
\hline \hline Same-Side (Kaon) Tag & 0.2 & 2.6 \\
Soft Lepton Tag $(\mu)$ & 1.0 & 1.0 \\
Soft Lepton Tag $(e)$ & 0.7 & 0.7 \\
Jet Charge & 3.0 & 3.0 \\
Opposite-Side Kaon Tag & - & 2.4 \\
\hline Total & $4.9 \%$ & $9.7 \%$ \\
\hline
\end{tabular}

Table 5.3: Projected values for $\epsilon D^{2}$ for Run II without and with TOF. These flavor tagging efficiencies are for $B_{s}^{0} / \bar{B}_{s}^{0} \rightarrow J / \psi \phi$. 


\begin{tabular}{|l|l|l|}
\hline \multicolumn{3}{|c|}{$B_{s}^{0} \rightarrow D_{s}^{-} \pi^{+}$} \\
\hline & without TOF & with TOF \\
\hline Same-Side (Kaon) Tag & 1.0 & 4.2 \\
Soft Lepton Tag $(\mu)$ & 1.0 & 1.0 \\
Soft Lepton Tag $(e)$ & 0.7 & 0.7 \\
Jet Charge & 3.0 & 3.0 \\
Opposite-Side Kaon Tag & - & 2.4 \\
\hline Total & $5.7 \%$ & $11.3 \%$ \\
\hline
\end{tabular}

Table 5.4: Projected values for $\epsilon D^{2}$ for Run II without and with TOF. These flavor tagging efficiencies are for $B_{s}^{0} \rightarrow D_{s}^{-} \pi^{+}$

\section{4 $B_{s}$ Mixing Performance with TOF and Layer 00}

We present the sensitivity to $B_{s}^{0}$ mixing using the baseline detector as defined in the TDR, and with TOF and Layer 00 added separately and then combined. TOF improves the flavor tagging efficiency, and Layer 00 the ability to resolve the oscillations.

The sensitivity is studied assuming a signal of 20,000 fully reconstructed $B_{s}^{0}$ decays obtained using the two decay channels $B_{s}^{0} \rightarrow D_{s}^{+} \pi^{-}$and $B_{s}^{0} \rightarrow$ $D_{s}^{+} \pi^{-} \pi^{+} \pi^{-}$. This number is based on a study of the expected performance of the Level 1 track trigger and the Level 2 secondary vertex trigger[35]. This is the number of events before flavor tagging.

A simple Monte Carlo program was used to estimate the sensitivity to $B_{s}^{0}$ oscillations. The proper time of $B_{s}^{0}$ decays was generated according to the distribution expected after impact parameter cuts imposed by the Level 2 secondary vertex trigger. The $B_{s}^{0}$ production flavor was assumed to be tagged correctly with probability $\mathcal{P}_{\mathrm{tag}}=2 D-1$, and the probability that the $B_{s}^{0}$ had mixed at the decay time $t$ was

$$
\mathcal{P}_{\text {mix }}(t)=\frac{1}{2}\left(1-\cos x_{s} t / \tau\right)
$$

where $\tau\left(B_{s}^{0}\right)=1.54 \mathrm{ps}$ [28]. After smearing with the proper time resolution, these decay times were entered into mixed and unmixed histograms. The mixed asymmetry, defined as

$$
A_{\text {mixed }}(t)=\frac{N_{\text {mixed }}(t)-N_{\text {unmixed }}(t)}{N_{\text {mixed }}(t)+N_{\text {unmixed }}(t)}
$$

was determined from the mixed and unmixed distributions. The asymmetry was fit to the function

$$
a_{\text {mixed }}(t)=-A \cos x_{s} t / \tau,
$$

where the amplitude $A$ is determined by the tagging dilution $(D)$, the proper 
time resolution $\left(\sigma_{t}\right)$, and the signal-to-background ratio $(S)$ :

$$
A=\frac{D e^{-\left(x_{s} \sigma_{t} / \tau\right)^{2} / 2}}{1+1 / S}
$$

The exponential term describes the expected effective dilution due to the smearing in proper time. We see that the reach in $x_{s}$ is inversely proportional to the proper time resolution.

The only free parameter in the fit was $x_{s}$; including the amplitude $A$ as a second free parameter did not reduce appreciably the $x_{s}$ reach. The difference between the deepest and next-to-deepest minima in the curve of the negative$\log$-likelihood $-\log \mathcal{L}$ as a function of $x_{s}$ was then used to define the significance of an $x_{s}$ measurement $N_{\sigma}=\sqrt{2 \Delta \log \mathcal{L}}$. An ensemble of simulated experiments was used to determine the average significance $\left\langle N_{\sigma}\right\rangle$ of the measurements at a specific value of $x_{s}$.

The combination of Layer 00 and TOF will increase substantially the range of values of $x_{3}$ for which we can obtain a significant measurement. This significance depends on the tagging efficiency, the dilution, the proper time resolution, and the background level according to

$$
N_{\sigma} \propto \frac{\sqrt{\epsilon D^{2}} e^{-\left(x_{s} \sigma_{t} / \tau\right)^{2} / 2}}{1+1 / S} .
$$

Any improvement in the proper time resolution, $\sigma_{t}$, translates into an extension of the range of $x_{s}$ for which measurements of a given significance can be made. The proper time resolution expected from SVX-II is about 60 fs while the addition of Layer 00 will improve this to $45 \mathrm{fs}$. Hence, Layer 00 increases the range of sensitivity to $B_{s}^{0}$ oscillations by over $30 \%$. Time-of-Flight has a direct impact on the significance at a particular value of $x_{s}$ in two ways. First, as shown in Table $5.4, \epsilon D^{2}$ is increased from $5.7 \%$ to $11.3 \%$ with the addition of opposite-side and same-side kaon tags. Second, the ability to identify kaons and use them in the $D_{s}^{+}$reconstruction will reduce the level of background under the $B_{s}^{0}$ mass peak.

The Monte Carlo study of $B_{s}^{0}$ mixing[37] included the effects of finite $B_{s}^{0}$ momentum resolution, the background level, and the presence of non-Gaussian tails in the proper time resolution function. Since the $B_{s}^{0}$ is fully reconstructed, the momentum resolution is expected to be $\Delta p_{T}\left(B_{s}^{0}\right) / p_{T}\left(B_{s}^{0}\right)<0.4 \%$ and has a negligible effect on the proper time resolution. The signal-to-background level $S$ is difficult to estimate. In Run I, CDF did not have an effective trigger for all-hadronic final states such as $B^{0} \rightarrow D^{*-} \pi^{+}$. We were successful in reconstructing an inclusive charm signal in data collected with an inclusive lepton trigger. In these events, one of the $B$ hadrons decayed semileptonically, and a fully reconstructed charmed hadron originated from the decay of the other $B$ $(B \rightarrow D X)$. Based on the signal-to-background level observed in these samples, we adopted a range of $S=1: 2$ to $S=2: 1$ for our studies of our sensitivity to 
$B_{s}^{0}$ oscillations. We also studied the effect of non-Gaussian tails in the proper time distribution. For our predictions shown here, we do not include these tails. Our experience with our Run-I data has shown that these tails can be reduced to a negligible level, if necessary, by applying strict enough selection criteria on the silicon clusters associated to the reconstructed tracks [38]. This results, of course, in some loss in statistics.

Figure 5.13 shows how the addition of Layer 00 and TOF extends the range of $x_{s}$ for which we would observe $B_{s}^{0}$ oscillations for two different values of signalto-background. For the baseline, we assume a combined $\epsilon D^{2}=5.7 \%$, and when TOF is included, we assume a combined $\epsilon D^{2}=11.3 \%$. The effects of momentum
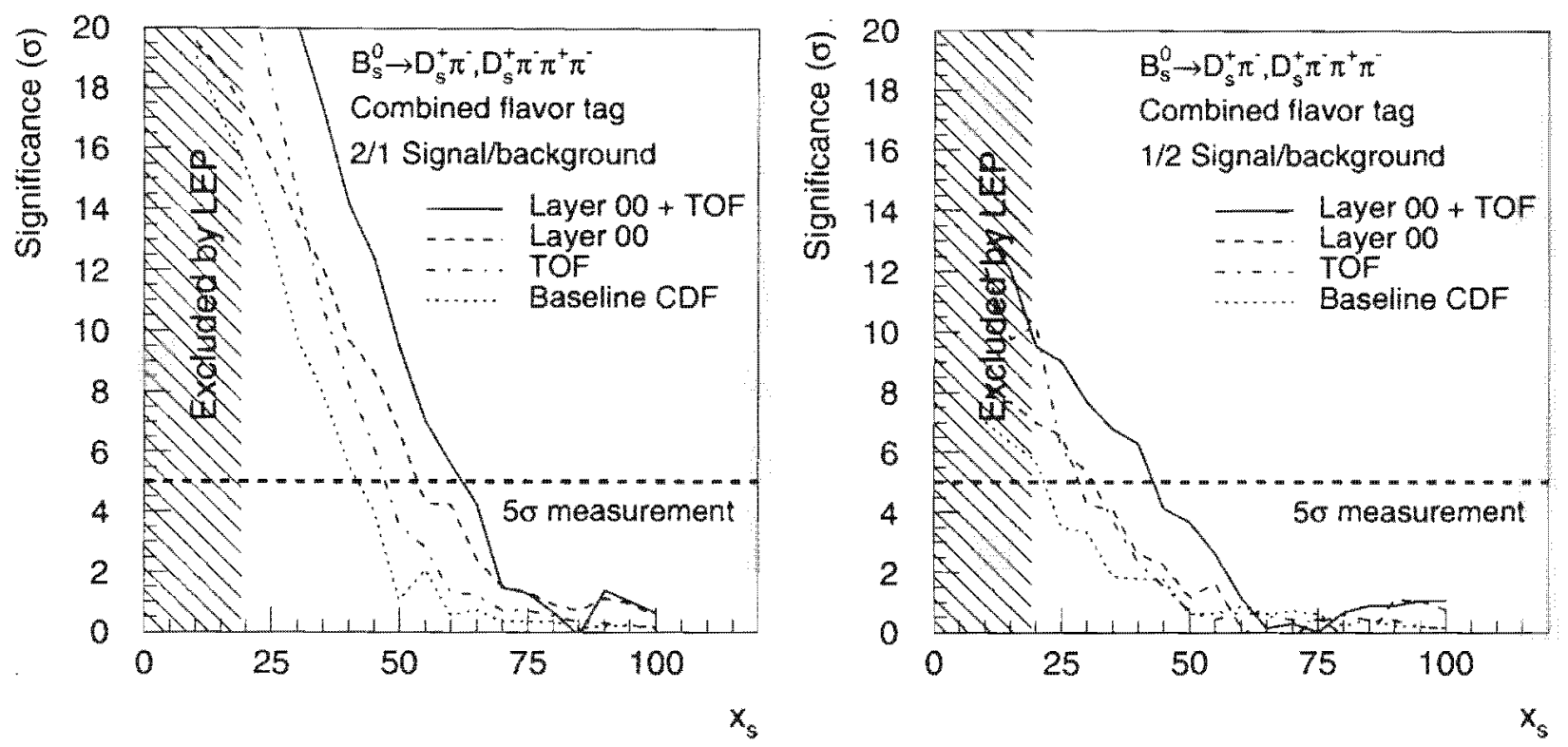

Figure 5.13: Average significance of $x_{3}$ measurements obtained with signal-tobackground ratios of $2: 1$ (left) and 1:2 (right).

resolution and non-Gaussian tails were neglected. In both cases, the $x_{s}$ reach is extended by about 20 with the addition of Layer 00 and TOF, allowing the range of $x_{s}$ preferred by the Standard Model to be covered even in the case of a 1:2 signal-to-background ratio. We extended this study by adding $5 \sigma$ non-Gaussian tails in the proper time distribution to $10 \%$ of the entries in the proper time distributions. This reduced the $x_{s}$ reach by about 5 .

Figure 5.14 shows how an observation of $B_{s}^{0}$ oscillations with $x_{s}=30$ and a signal-to-noise of 1:2 might appear if performed with the baseline CDF detector and with the addition of Layer 00 and TOF. The significance of these two observations is shown in Figure 5.15 by plotting the $-\log \mathcal{L}$ as a function of $x_{s}$. There is a local minimum at $x_{s}=30$ with the baseline detector, but one could not claim an observation with this distribution since a deeper minimum exists at $x_{s}=59$ with a significance of $2 \sigma$. With the Layer 00 and TOF upgrades, however, $x_{s}$ is measured to be $29.94 \pm 0.06$ with a significance of 11 standard 

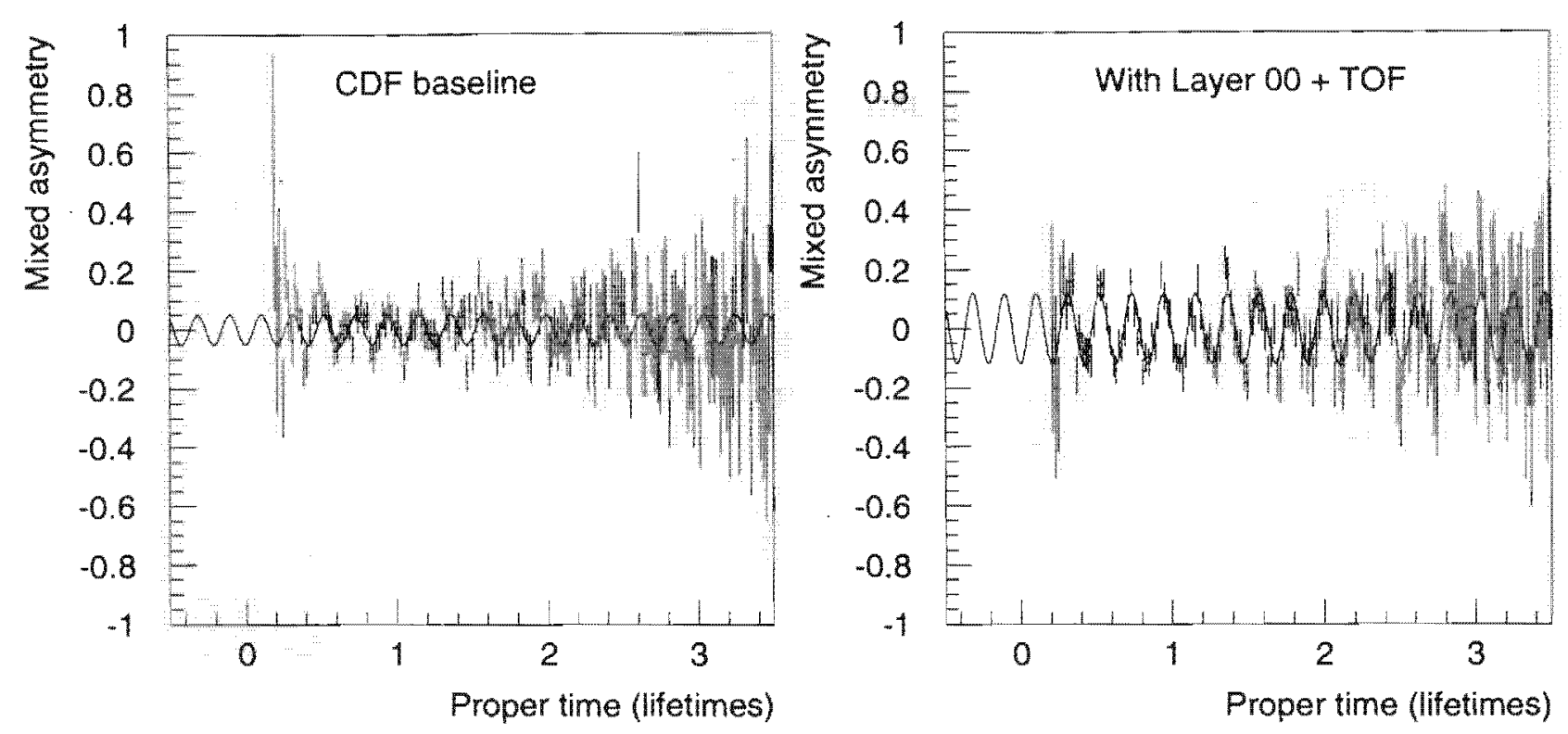

Figure 5.14: An examples of the mixed asymmetry vs proper time for $x_{s}=30$ for the baseline CDF detector (left) and with the addition of Layer 00 and Timeof-Flight (right). The signal-to-background ratio in this example is 1:2.

deviations.

Even if a particular value of $x_{s}$ yields a $5 \sigma$ measurement on average, there can still be a high probability that no significant measurement can be made. For example, for $x_{s}=30$ and a signal-to-background ratio of $1: 2,25 \%$ of $x_{s}$ measurements have significance of less than $3 \sigma$ with the baseline detector. With the additions of Layer 00 and TOF, this percentage is reduced to $5 \%$. Alternatively, the reach can be defined as the value of $x_{s}$ where a measurement with a significance of at least $5 \sigma$ can be made $95 \%$ of the time. Adopting this definition lowers all of our $x_{s}$ reach projections by about 10 .

We have used $B_{s}$ mixing as the benchmark process to demonstrate the power of the TOF and Layer 00 upgrades. It is difficult to determine with high certainty the reach in $x_{s}$ of the baseline detector because we lack the knowledge for such important quantities as signal-to-background of the $B_{s}$ mass peak. We find over a broad range of assumptions, however, that CDF-II will benefit dramatically from TOF and Layer 00. In all cases considered here, the differential improvement, relative to the baseline detector, is significant. The improvement will be greater if Time-of-Flight is used to reduce backgrounds by identifying the kaons in the final state. The improvement in $\epsilon D^{2}$ can also be considered insurance against the possibility that strict selection criteria may be required either to reduce the levels of background or non-Gaussian tails in the proper time resolution function. 

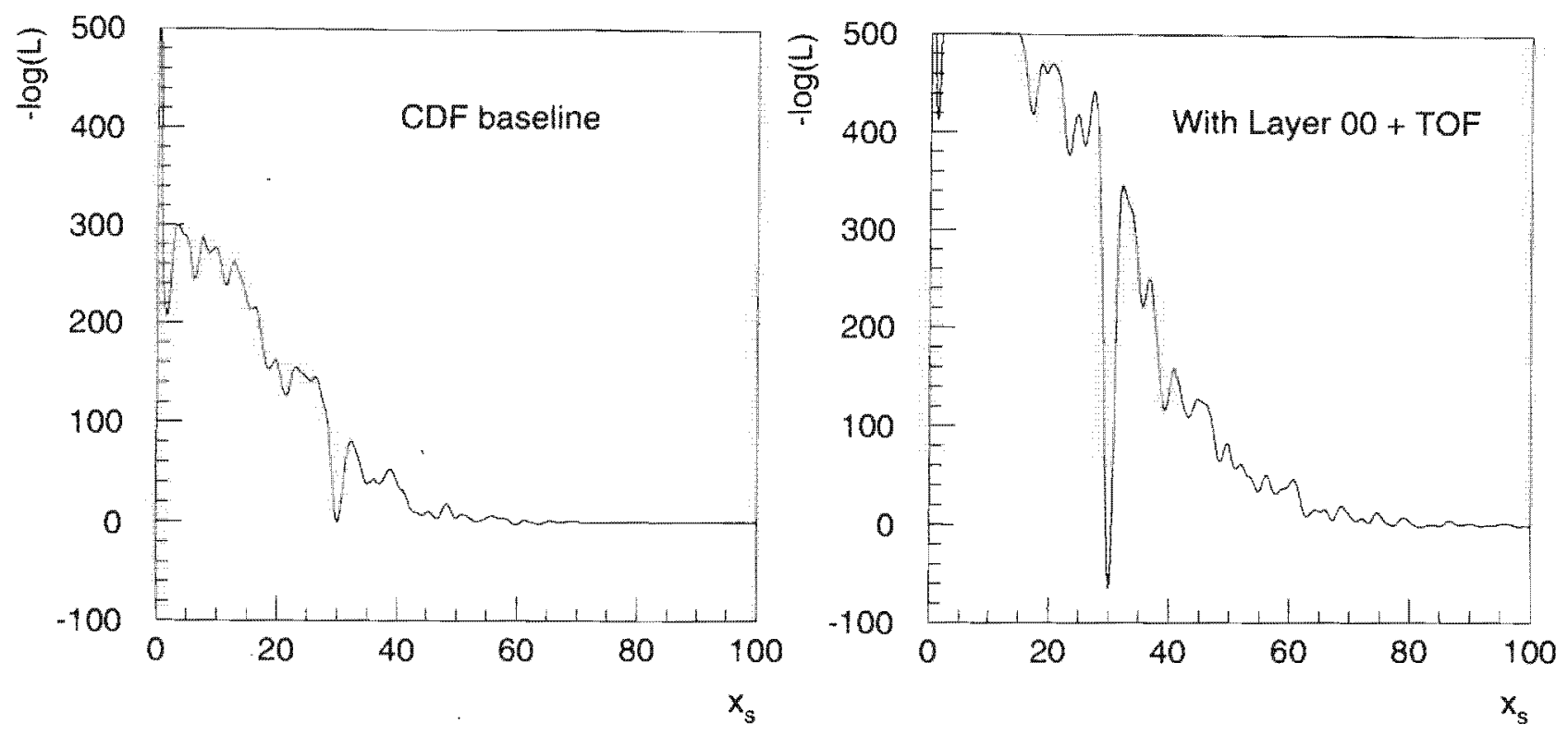

Figure 5.15: A comparison of $-\log \mathcal{L}$ vs $x_{s}$ when $x_{s}=30$ for the baseline detector (left) and proposed upgrade scenarios (right).

\subsection{Search for CP asymmetries in the $B_{s}$ system}

Even if an observation of $B_{s}$ oscillations is well within our reach, there are still strong motivations to optimize our ability to resolve these oscillations, since certain interesting CP asymmetries are modulated by the oscillation frequency. The CP asymmetry in the decay mode $B_{s} \rightarrow D_{s} K$ can be used to measure the $\mathrm{CP}$ angle $\gamma$. The CP asymmetry in $B_{s} \rightarrow J / \psi \phi$ provides a test of the unitarity of the CKM matrix in a single measurement. We expect (to first order) a CP asymmetry in this decay mode if and only if the unitarity triangle doesn't close. Such an asymmetry would signal an anomolous CP violating phase in the $b \rightarrow s$ transition. Y. Nir [40] discusses scenarios which can result in such a phase.

An early analysis of our reach for the CP angle $\gamma$ in the decay mode $B_{s} \rightarrow$ $D_{s} K[39]$ assumed a very pessimistic flavor tagging efficiency. If we simply rescale the results of this analysis for the flavor tagging efficiency shown in Table 5.4 and $2 \mathrm{fb}^{-1}$ of data, we obtain an uncertainty on $\sin \gamma$ of 0.25 . Thus, with the TOF and Layer 00 upgrades, we can begin to be sensitive to the angle $\gamma$ in Run II.

Our Run I experience is that our yield of $B_{s} \rightarrow J / \psi \phi$ events will be about $60 \%$ that of $B_{d} \rightarrow J / \psi K_{S}$. We assume that this ratio will also apply in Run II. The expected flavor tagging efficiencies are shown in Table 5.3. An angular analysis may be necessary to separate the different possible CP eigenstates contributing to this final state, and we neglect the loss of sensitivity from this procedure. With these assumptions, the uncertainty in the CP asymmetry for $B_{s} \rightarrow J / \psi \phi$, as a function of assumed $x_{s}$, is shown in Fig. 5.16. We see that with the Layer 


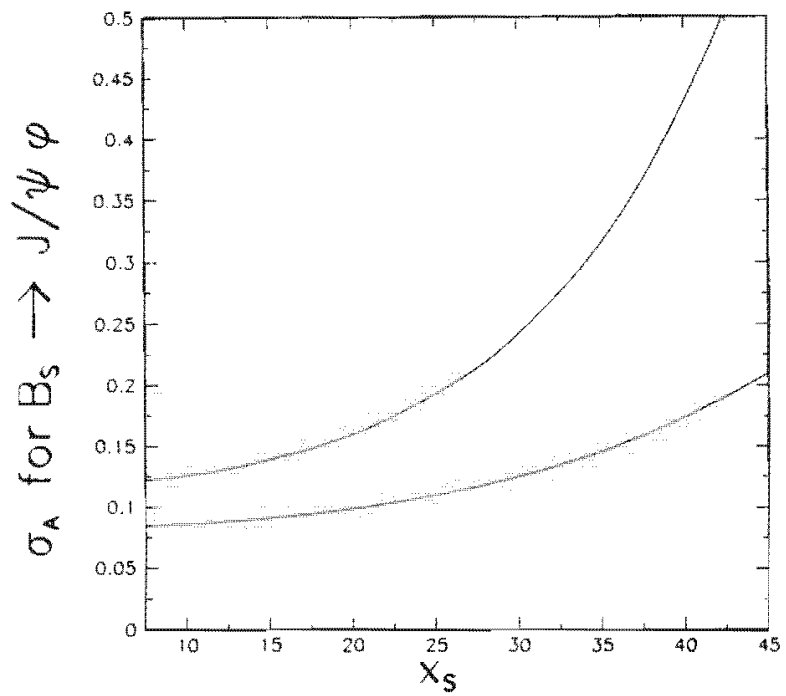

Figure 5:16: The uncertainty on the CP asymmetry for $B_{s} \rightarrow J / \psi \phi$ as a function of the $B_{s}$ mixing parameter $x_{s}$. The top curve is for the baseline CDF II, and the bottom curve includes improvements from TOF and Layer 00 .

00 and TOF upgrades, our sensitivity is improved, and falls off more slowly with increasing $x_{s}$.

\subsection{Sensitivity to Gauge-Mediated Supersymme- try}

The supersymmetric model we use is one in which the stau is the NLSP. With these parameters, the stau is the next-to-lightest SUSY particle and decays to a $\tau$ and a gravitino on a long time scale. The result is that all SUSY particles produced decay to the stau which is observed as a heavy charged particle in the CDF-II detector. Since SUSY particles are produced in pairs, we expect two of these objects in each event.

A TOF system will extend the CDF-II stau limit sensitivity by about 50 $\mathrm{GeV} / c^{2}$ from $\sim 150 \mathrm{GeV} / c^{2}$ to $\sim 200 \mathrm{GeV} / c^{2}$. A $4 \sigma$ discovery sensitivity would reach about $160 \mathrm{GeV} / c^{2}$ with TOF, and about $110 \mathrm{GeV} / c^{2}$ without the TOF system. The momentum acceptance for a $100 \mathrm{GeV} / c^{2}$ SUSY $\tau$ is shown in Figure 5.17. 


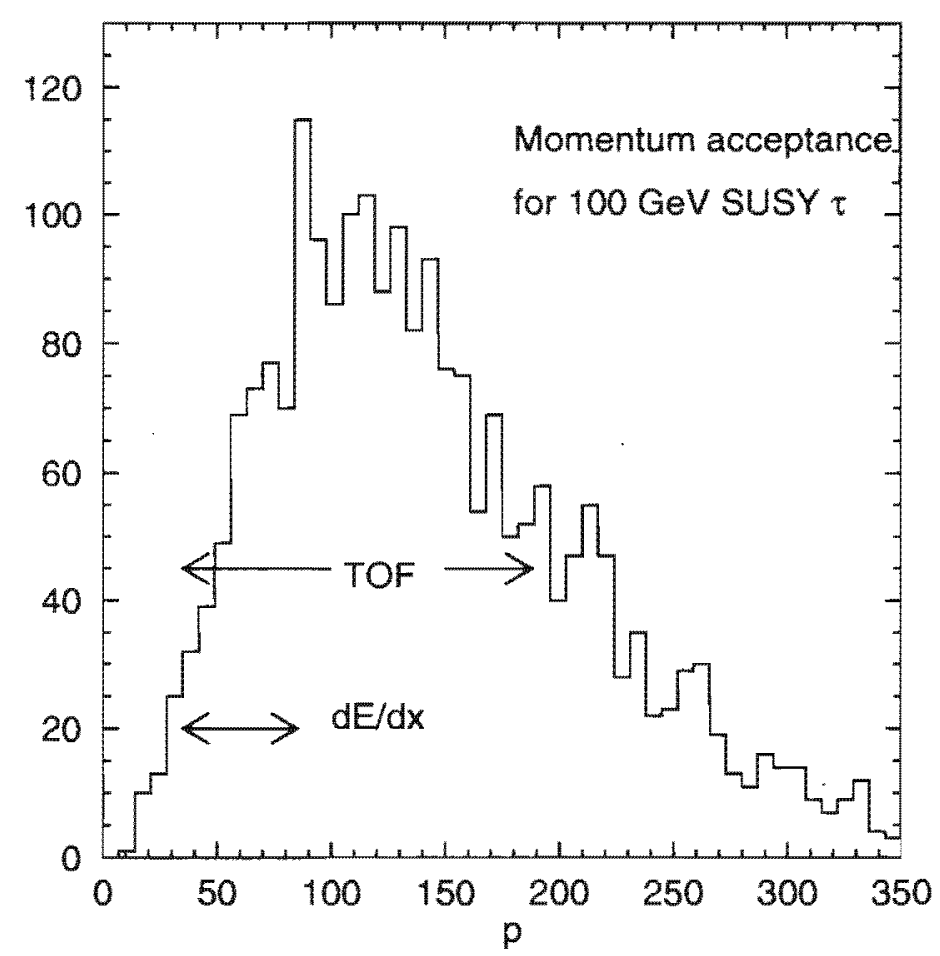

Figure 5.17: The large increase in momentum acceptance with the proposed time-of-flight system installed in CDF-II. 


\subsection{Other Potential Uses for the TOF System}

The TOF system has other potential uses besides $\pi / K / p$ identification. We have not explored any of these options in detail, but merely point out the possibilities. Several of these are presently under study.

- TOF could be used to reject cosmic ray muons in collider events.

- It will be useful for making a cosmic ray trigger.

- It could be used to trigger on very highly ionizing objects, such as monopoles.

- It could serve as a preradiator in front of the coil, since we measure pulse height.

- The TOF system can be used as a luminosity monitor. It is well known from Run-I that independent cross checks of the luminosity are very important for understanding the associated systematic error.

- TOF information can be combined with the XFT track processor and calorimetry information as part of a level 2 "mass" trigger.

\subsection{Summary}

$B$ flavor tagging is a crucial experimental component in the measurement of $C P$ asymmetries of the decays of neutral $B$ mesons to $C P$ eigenstates and in the measurement of neutral $B$ meson flavor oscillations. In particular, the search for $B_{s}^{0} \leftrightarrow \vec{B}_{s}^{0}$ flavor oscillations, the measurement of the asymmetry between the decays $B_{s}^{0} / \bar{B}_{s}^{0} \rightarrow J / \psi \phi$, which is a sensitive probe of physics beyond the Standard Model, and the measurement of the asymmetry between the decays $B^{0} / \bar{B}^{0} \rightarrow J / \psi K_{\mathrm{S}}^{0}$, which determines $\sin 2 \beta$, will be among the most important measurements in $B$ physics made during the first years of the next decade. CDF is in the unique position to study all three phenomena. We have found that the TOF and Layer 00 are particularly important for our studies involving $B_{s}^{0}$ mesons.

To determine the additional benefits of adding TOF, we compare to the results we achieved in $B$ flavor tagging in Run $I$ and to our projected $B$ flavor tagging in Run II without TOF. We have quantified the performance of the flavor tags using their effective tagging efficiency, $\epsilon D^{2}$. If we have a sample of $N$ events, then the statistical precision with which we can determine an asymmetry or the statistical significance with which we can measure an oscillation frequency is approximately inversely proportional to $\sqrt{\epsilon D^{2} N}$. If we can add a flavor tagging technique or techniques that double the value of $\epsilon D^{2}$, we have effectively doubled the statistics of our data sample. 
Using our Run I data, we measured $\epsilon D^{2}$ of three flavor tagging techniques: the soft-lepton tag (SLT), the jet-charge tag (JCT), and the same-side tag (SST). The measured values of $\epsilon D^{2}$ are $\epsilon D^{2}(\mathrm{SLT})=1.07 \pm 0.13 \%, \epsilon D^{2}(\mathrm{JCT})=$ $0.78 \pm 0.15 \%$, and $\epsilon D^{2}(\mathrm{SST})=1.8 \pm 0.4 \%$, for a total of $\epsilon D^{2}$ (Combined) $=3.7 \%$. This combined number is applicable to tagging $B^{0}$ decays only, since SST was measured for $B^{0}$ (SLT and JCT are opposite-side tagging techniques so we assume they apply equally to $B^{0}$ and $B_{s}^{0}$ ).

In Run II, improvements to the detector are expected to enhance significantly the effectiveness of our existing favor tags: for the SLT, we expect $\epsilon D^{2}$ (SLT) $=$ $1.7 \%$, and for JCT, our projection is $\epsilon D^{2}(\mathrm{JCT})=3.0 \%$. These projections may be found in the the CDF Run II TDR. For $B^{0}$, the projected flavor tagging effectiveness for SST is $\epsilon D^{2}(\mathrm{SST})=2.0 \%$, without TOF, yielding a total of $\epsilon D^{2}$ (Combined) $=6.7 \%$. For $B_{s}^{0}$, the projected flavor tagging effectiveness for $\mathrm{SST}$ is $\epsilon D^{2}(\mathrm{SST})=1.0 \%$, without TOF, yielding a total of $\epsilon D^{2}($ Combined $)=$ $5.7 \%$.

We find that for opposite-side kaon tagging (OKT), $\epsilon D^{2}(\mathrm{OKT})=2.4 \pm 0.02 \%$ (the error is the statistical error only). Opposite-side kaon tagging can be applied equally to $B^{0}$ and $B_{s}^{0}$. For SST in $B^{0}$, we find that using TOF to help select only pions as SST candidates improves $\epsilon D^{2}$ by $30 \%$. For SST in $B_{s}^{0}$, we find that using TOF to help select only kaons as SST candidates yields values of $\epsilon D^{2}$ ranging from $3 \%$ to $5 \%$ depending on $\left\langle p_{T}(B)\right\rangle$ of the data sample. This method alone would more than double $\epsilon D^{2}$ for $B_{s}^{0}$ tagging from the value we achieved so far in Run I (1.9\% from SLT and JCT combined), and could end up being the most powerful of all our flavor tagging techniques. Combining all our flavor tags, and including those made possible by TOF we could tag $B_{s}^{0}$ mesons with a combined $\epsilon D^{2}$ (Combined) $=9.7 \%$ to $11.3 \%$.

The summary of our findings is

- TOF would make a dramatic difference in our ability to study the $B_{s}^{0}$ system: it doubles our flavor tagging effectiveness compared to what we expect without TOF in Run II. This is especially important given the uniqueness of this physics to the Tevatron and the crucial role it plays in the test of closure of the unitarity triangle.

- Same-side kaon tagging for $B_{s}^{0}$ using TOF may be our most powerful $B$ flavor tag.

- The combination of TOF and Layer 00 will lead to a substantial increase in our sensitivity to $B_{s}^{0}$ oscillations. The value of $x_{s}$ for which a measurement could be made with an average significance of at least $5 \sigma$ would be extended from $x_{s}=45$ to $x_{s}=65$.

- TOF will also improve our tagging of $B^{0}$ : the total flavor tagging effectiveness increases from $6.1 \%$ without TOF to $9.0 \%$ with TOF. This corresponds to an increase of $40 \%$ of the statistics of our $J / \psi K_{\mathrm{S}}^{0}$ sample for the measurement of $\sin 2 \beta$. 
- The TOF system will allow the search for the supersymmetric tau well beyond the LEP-II limits. TOF extends the discovery reach for stau from $110 \mathrm{GeV} / c^{2}$ to $160 \mathrm{GeV} / c^{2}$.

Having begun the process of studying the application of TOF at CDF-II, we have not had time to study all the potential uses of the system, and if approved, the TOF will be a very important and productive part of the many different analyses not yet studied. 


\section{Chapter 6}

\section{Conclusions}

Technical descriptions of the Layer 00 silicon detector and the TOF detector have been presented in Chapters 2 and 3 , and enhancements to the physics capabilities of the CDF II research program have been described in Chapters 4 and 5 . In this chapter we briefly consider implementation issues for these two proposals. We discuss prospects for resources and conclude with a physics summary.

\subsection{Implementation Issues}

Both Layer 00 silicon and the TOF system were reviewed by an internal CDF II committee appointed for this purpose. Based upon this evaluation process, we are convinced that these proposals are technically sound, and will provide important extensions of the physics capabilities of the CDF II detector. Part of the charge to this committee was to evaluate possible impact of increased scope on the timely completion of the baseline detector. Obviously any increase in the scope of the baseline upgrade project carries with it some risk of delaying the project completion date. We propose to minimize this risk through a combination of staging strategies and addition of new collaborators. We describe below these ideas for each of the two proposed upgrades. We emphasize that this is work in progress. In particular, development of Layer 00 staging strategies is ongoing. We include preliminary cost estimates, but note that these have not yet been reviewed in detail. In addition, negotiations with new groups are ongoing to secure funding and personnel. Nevertheless, we believe that the information available now is useful to show the scale of the increase in scope we are proposing, and the progress we are making toward identifying strategies and resources for pursuing these upgrades.

\subsubsection{Silicon Layer 00}

Silicon Layer 00 will have about 16,000 channels. This represents only a few per cent increase in the CDF II upgrade channel count for silicon. Layer 00 
would use the same readout chip (SVX3) as other CDF II silicon systems. All electronics from the port card onward would also be identical to those used in other CDF II silicon systems. Thus no development effort is required for any of these components.

The development effort required for Layer 00 may be divided into three areas - the sensors, hybrids and mechanical/cooling issues. These have been discussed in section 2.3. Hybrid development is expected to be done at LBNL, where both the SVX II and ISL hybrids are being designed.

The sensor development and testing is expected to be done by new collaborators. We are now negotiating with groups from the UK to join the CDF II silicon effort. These groups are planning to contribute people to the baseline silicon projects (especially the readout and software areas) and are also very interested in the Layer 00 proposal. The possibility of gaining experience with LHC-style radiation-hard sensors is very attractive to these groups.

Currently we envision the bulk of the mechanical work being done at FNAL, though at least one UK group has indicated that they have engineering and design support that could be made available to CDF II. The interaction of this work with the baseline silicon projects has not yet been worked out in detail.

An estimate of the material cost for Layer 00 is shown in Table 6.1. We expect that spare SVX 3 chips would be used. Use of spare DOIMs is also possible. These would provide some cost savings.

A requirement of Layer 00 is that it not delay the baseline silicon projects. This can be accomodated by staging the installation, if necessary, though we plan to complete the installation at the start of Run II. While allowing for a staged installation complicates the mechanical design, it insures that Layer 00 will be compatible with the baseline upgrade project. Furthermore, such a design is desirable to allow swapping out the silicon and readout chips, in situ, to allow for extended running.

The schedule for Layer 00 and the impact on Run II are currently under study in conjunction with efforts to secure additional resources from outside (new groups) and within the collaboration. Support of the PAC and Stage I scientific approval from the Director will help us to acquire non-U.S. resources. Our plan would be to present more complete funding and schedule information to the Fermilab Director as soon as possible and certainly prior to requesting stage II approval. We believe that Layer 00 will provide a significant increase in physics capability for a relatively modest cost.

\subsubsection{TOF System}

The effort required to construct and install the TOF system for Run II can be well estimated from our experience with the 20 bar prototype TOF system tested in Run Ic. In addition, this same experience allows us to state with confidence that the TOF system proposed for Run II can be staged and can be scheduled 


\begin{tabular}{|l|r|r|r|}
\hline & $\#$ & Unit Cost & Total Cost \\
\hline \hline DAQ Items & 128 & 150 & 19,200 \\
\hline SVX3D & 32 & 500 & 16,000 \\
HDI & 8 & 1200 & 9,600 \\
Portcard & 32 & 1500 & 48,000 \\
DOIMs & 32 & 500 & 16,000 \\
DOIM fibers & 4 & 6550 & 26,200 \\
FIB & 4 & 5500 & 22,000 \\
VRB & 16 & 766 & 12,256 \\
GLinks & 8 & 8000 & 64,000 \\
Power supply & & & 233,256 \\
\hline crate slots) Total DAQ: & \multicolumn{3}{|c|}{} \\
\hline \hline Non DAQ items & 64 & 500 & 32,000 \\
\hline Silicon & 128 & 500 & 64,000 \\
Signal Cables & 128 & 600 & 76,800 \\
Hybrid & & & 35,000 \\
Mechanical & & & 207,800 \\
\hline Total NonDAQ:
\end{tabular}

Table 6.1: Estimated cost of Layer 00

such that the risk of delay to the baseline schedule is minimized. The only real requirements for these assertions to be true is that the scintillator bars and mounts be purchased and installed by the Fall of $1999 .^{1}$

The planned scintillator bars, light collectors, and PMT's will be similar to those already constructed. We understand their performance and no new R\&D is required. The scintillator bars, light concentrators, and PMT mounts will all be ordered commercially. The assembly time can be short. We estimate that one physicist and two technicians can assemble, wrap, test and install the scintillator bars in nine months. A small amount of additional engineering and drafting will be needed for the mounts. Resources for this work could be available on the required time schedule as the COT mechanical work starts to wind down. Photomultiplier testing, cables, and the bulk HV system for the TOF system will require another FTE physicist for one FTE year.

One long lead time item is the fine mesh PMT's from Hamamatsu. The TOF test system employed a 16 stage tube. This tube could be used for the proposed system, however, our tests indicate a 19 stage tube would provide higher gain and thus better performance due to better noise immunity. Hamamatsu has already made 19 stage 2 inch diameter tubes for KEK. They indicated to us that they can make 100 of our desired 1.5 inch round 19 stage tube with a lead

\footnotetext{
${ }^{1}$ The TOF could still be staged without the scintillator bars installed at the beginning of the run. However, in this case installation of the scintillator bars in situ would require that they be less than $1.5 \mathrm{M}$ in length and this would double the number required. This in turn would substantially increase the cost by doubling the number of readout channels.
} 
time of four months and then deliver 100 tubes/month afterwards. To avoid this lead time it would be attractive to order some of these tubes soon. However, we have developed a scheme that would allow us to mount PMT's in situ provided the scintillator bars are already installed. The PMTs could be installed during any two day or longer access in which we open the endplugs. Similarly although it is desirable to install the TOF cables through the 30 degree crack early, they too could be added at a later date.

The electronics for the system will be new and custom, but will be a variant of electronics used to read out the CDF II calorimeters in Run II, as described in section 3.3. The electronics development, production and installation could all be staged as necessary. The electronics infrastructure for TOF is straight forward. We have reserved extra slots in existing endwall VME crates to house the TOF cards such that there is no cost or effort associated with new crates or power supplies.

The TOF R\&D activities for Run Ic were carried out by the University of Pennsylvania, Fermilab and the University of Tsukuba. The University of Pennsylvania is expected to lead this project and has several people committed to it. In particular, the Penn group plans to do the electronics development, in consultation with Fermilab experts on the ADMEM boards. The electronics development would be done after Penn and Fermilab engineers had finished their baseline jobs (COT and calorimeter electronics). There is interest in the project from other U.S. and non-U.S. CDF II collaborators as well.

An estimate for the material cost for the TOF system is shown in Table 6.2 Installation of the scintillator before Run II begins would require about $\$ 200 \mathrm{~K}$. We are exploring possibilities of obtaining this funding from non-U.S. collaborators. 


\begin{tabular}{|c|c|c|c|c|c|}
\hline Component & Quantity & Cost & Basis & Total & Summary \\
\hline \multicolumn{6}{|l|}{ Detector } \\
\hline PMTs & $432(450)$ & $\$ 1037$ & Quote & $\$ 466650$ & \\
\hline PMT Testing & $450(450)$ & $\$ 33$ & Quote & $\$ 14850$ & \\
\hline $\mathrm{Ba} 1$ se & $432(450)$ & $\$ 12$ & Eng. est. & $\$ 5400$ & \\
\hline Preamp & $432(450)$ & $\$ 16$ & Eng. est. & $\$ 7200$ & \\
\hline PMT Mech. Mount & $432(450)$ & $\$ 30$ & Eng. est. & $\$ 13500$ & \\
\hline Scintillator & $216(225)$ & $\$ 760$ & Quote & $\$ 171000$ & \\
\hline Scin. Mech. Mount & $24(25)$ & $\$ 0$ & in baseline & $\$ 0$ & $\$ 687,600$ \\
\hline \multicolumn{6}{|l|}{ Electronics } \\
\hline Unmodified ADMEM & $24(26)$ & $\$ 3500$ & FNAL & $\$ 91000$ & \\
\hline DECAF & $432(450)$ & $\$ 100$ & Eng. est. & $\$ 45000$ & \\
\hline Modified ADMEM & $24(28)$ & $\$ 3700$ & Eng. est. & $\$ 103600$ & \\
\hline Cent. Clock Dist. & $1(2)$ & $\$ 4500$ & Eng. est. & $\$ 9000$ & \\
\hline Clock Fanout & $8(12)$ & $\$ 850$ & Eng. est. & $\$ 10200$ & \\
\hline Board Testing & $488(488)$ & $\$ 40$ & Eng. est. & $\$ 19520$ & \\
\hline Syst. Test/Integ. & $1(1)$ & $\$ 5000$ & Eng. est. & $\$ 5000$ & $\$ 330,775$ \\
\hline \multicolumn{6}{|c|}{ Calibration and Monitoring } \\
\hline Laser & $1(1)$ & $\$ 22000$ & Eng. est. & $\$ 22000$ & \\
\hline Fibers \& Connect. & $216(225)$ & $\$ 35$ & Eng. est. & $\$ 7875$ & \\
\hline Monitor \& Control & $1(1)$ & $\$ 7000$ & Eng. est. & $\$ 7000$ & $\$ 36,875$ \\
\hline \multicolumn{6}{|l|}{ Power Supplies } \\
\hline HV Bulk + "COW" & $1(1)$ & $\$ 9500$ & Eng. est. & $\$ 9500$ & \\
\hline LV for preamp & $8(10)$ & $\$ 650$ & Eng. est. & $\$ 6500$ & $\$ 16,000$ \\
\hline \multicolumn{6}{|l|}{ Cables } \\
\hline PMT Coax to ADMEM & $432(450)$ & $\$ 16$ & Eng. est. & $\$ 7200$ & \\
\hline PMT HV to HV Syst. & $432(450)$ & $\$ 18$ & Eng. est. & $\$ 8100$ & \\
\hline PMT LV to LV Syst. & $432(450)$ & $\$ 10$ & Eng. est. & $\$ 4510$ & \\
\hline Timing, Cent. to Crate & $16(20)$ & $\$ 40$ & Eng. est. & $\$ 800$ & \\
\hline Timing, Fanout to Cards & $48(55)$ & $\$ 30$ & Eng. est. & $\$ 1650$ & $\$ 23,250$ \\
\hline \multicolumn{5}{|l|}{ Total Cost } & $\$ 1,094,500$ \\
\hline
\end{tabular}

Table 6.2: Estimated cost of the Time-of-flight Detector. The numbers in parentheses in the second column indicate the quantity including spares. The cost of the spares is included in the total cost. 


\subsection{Resources for the Layer 00 and TOF upgrades}

In order to implement the Layer 00 and TOF projects we need to identify physicist and technical personnel beyond those required for completion of the baseline CDF II detector. In addition the funding required for early stages of these projects should not rely upon the release of contingency from the baseline upgrade project. Although these are difficult constraints, we believe they do not prohibit us from adding the Layer 00 and TOF detectors to the CDF II project. Our collaboration is convinced that the importance of these beyond-the-baseline detectors will allow us to generate the added resources required for their installation early in Run II.

We are pursuing two general approaches for obtaining the personnel and funding required to carry out the Layer 00 and TOF projects. The first is by adding new institutions to the Collaboration which can bring in additional resources. In recent months we have received proposals from four United Kingdom, three Korean, and one Russian institution to participate in the CDF II upgrade project. A collaboration requirement on new groups is that they contribute to the baseline upgrade, but, as has been indicated in Section 6.1, there is also significant interest in providing personnel and funding for the Layer 00 project. The United Kingdom institutions are requesting support from their HEP funding agencies, but at this time we can not predict the outcome of these initiatives. It is clear however that scientific endorsement of these upgrades by the Fermilab PAC would greatly enhance the prospects of obtaining this non-U.S. funding support.

The second approach to obtaining additional resources is through institutions already part of the CDF II Collaboration. The Collaboration has clearly established the completion of the baseline CDF II upgrade as highest priority. Both the Layer 00 and TOF proposals originated from physicists actively working on the baseline CDF II upgrades and dedicated first to their on-time completion. In some cases, resources will become available as institutional commitments to the baseline project are fulfilled. Support of the Layer 00 project has come from physicists at Fermilab and LBNL, and for the TOF project from the University of Pennsylvania, Fermilab, University of Tsukuba and Italian institutions. This is not an inclusive list of CDF II institutions interested in these projects but is indicative of the support we have from within the CDF Collaboration.

In summary we are aggressively pursuing support for the implementation of the Layer 00 and TOF detectors. We are acutely aware that our efforts must not impact the timely completion of the baseline CDF II detector. We do not have a resource loaded schedule and funding plan in place at this time, but are confident that these can be developed once the projects are granted Stage I scientific approval. 


\subsection{Summary of Physics Benefits of Layer 00 and TOF upgrades}

In this proposal we have described two complementary, new detectors which significantly improve the 'beauty' detection capabilities of the CDF II detector. This not only enhances our $B$ physics program, but also top quark studies and searches for new phenomena. We briefly summarize some of the physics benefits of the Layer 00 silicon and TOF detectors here. Details have been presented in Chapters 4 and 5 .

By providing a very precise tracking measurement exceptionally close to the beamline, Layer 00 substantially enhances the vertex resolution of the CDF II tracking system. This will benefit a wide variety of high $p_{t}$ physics studies, most notably those that benefit from double b-tagging, such as physics with the top quark and search for a Higgs boson decaying to $b \bar{b}$. We estimate that with Layer 00 our double b-tagging efficiency will improve from $21 \%$ to $28 \%$, and furthermore, will remain at $28 \%$ even as the innermost layer of the SVX II detector loses functionality from radiation damage. We expect to obtain further improvements in the double $b$-tagging efficiency from optimizations specifically designed to take full advantage of the improved resolution of Layer 00. Given the importance of the physics accessible via double $b$-tagging, it is crucial that we maximize the capabilities of the CDF II detector in this area.

With the addition of Layer 00, the vertex resolution of CDF II will compare very favorably with those of other experiments. With a CCD pixel layer at a radius of $2.8 \mathrm{~cm}$ from the beamline, the SLD experiment has achieved impressive results in the analysis of the vertex topology of their events [41]. With Layer 00 at $1.6 \mathrm{~cm}$, CDF II will have somewhat better resolution in the $r-\phi$ view and a somewhat worse resolution in the $r-z$ view, and can anticipate similar capabilities in topological analysis. The BTeV experiment, with multiple planes of silicon in the forward region, anticipates achieving very good proper time resolution for $B$ hadrons [42]. With Layer 00, CDF II will match the expected proper time resolution of $\mathrm{BTeV}$.

By providing for particle identification, in particular $K-\pi$ separation, the Time of Flight system, in conjunction with Layer 00 , will further enhance the $B$ physics capabilities of the CDF II detector. This is particularly the case for $B_{s}$ physics: $B_{s}$ mesons are produced in association with kaons useful for flavor tagging if they can be identified. Also $B_{s}$ final states are enriched in kaons, which a TOF system can potentially exploit to reduce backgrounds. We have taken as a benchmark our sensitivity to the $B_{s}$ mixing frequency $x_{s}$. With flavor tagging improvements from TOF, and the improvement in proper time resolution from Layer 00 , we expect our reach in $x_{s}$ to improve from 45 to 65 . This measurement, using fully reconstructed hadronic $B_{s}$ decays, relies on the ability of CDF II to trigger on tracks with nonzero impact parameters.

The proposed upgrades will also significantly enhance CDF II's ability to 
search for $\mathrm{CP}$ violation in $B_{s} \rightarrow J / \psi \phi$ decays as well significantly improve CDF II's competitive projections for measuring CP violation with $B_{d} \rightarrow J / \psi K_{S}$. We note that physics measurements with all species of $b$ hadrons, including the $B_{s}, B_{c}$ and $\Lambda_{b}$, are possible at the Tevatron, in contrast to $e^{+} e^{-}$collider physics at the $\Upsilon(4 s)$.

In summary, the additon of the silicon Layer 00 and the TOF upgrades will leverage the capabilities of the baseline CDF II detector and provide a substantial enhancement of our Run II physics program. The experience gained from operation of the inner layer of radiation-hard silicon could also lead to a solution for extending the lifetime of the CDF II silicon tracking system for the exciting physics potential of Tevatron Run III. 


\section{Bibliography}

[1] "The CDF II Detector: Technical Design Report", R. Blair, et al. (The CDF II Collaboration), Fermilab-PUB-96/390-E, November, 1996.

[2] "Measurement of the $C P$-Violation Parameter $\sin (2 \beta)$ in $B_{d}^{0} / \bar{B}_{d}^{0} \rightarrow J / \psi K_{S}^{0}$ Decays", The CDF Collaboration, F. Abe et al, FERMILAB-PUB98/189-E, submitted to Physical Review Letters.

[3] "Study of CDF II for TeV33", D. Amidei, A. Byon-Wagner, W. Foster, J. Huston, N. Lockyer, A. Menzione, P. Tipton. CDF Note 4370, December, 1997.

[4] "A Time-of-Flight System for CDF", CDF Note 2573, submitted to the PAC in April, 1994.

[5] I. Dunietz and J. Rosner, Phys. Rev. D34, 1404 (1986).

[6] "Measurement of the $B_{d}^{0}-\vec{B}_{d}^{0}$ oscillation frequency and study of same side flavor tagging of $B$ mesons in $p \bar{p}$ collisions", The CDF Collaboration, F. Abe et al., Phys. Rev. Lett. 80, 2057 (1998); and FERMILAB-PUB-98/188-E, submitted to Physical Review D.

[7] B. Hyams et al, NIM 205 (1983) 99.

[8] CDF Collaboration, CDF Upgrade Technical Design Report, CDF Note 3777 (1996).

[9] M. Frautschi, Radiation Damage Issues for the SVXII Detector, CDF Note 2368 (1994).

[10] J. Matthews, Effective Si Damage Fluences Measured by SVX and SVX', CDF Note 3937 (1996).

[11] J. Conway et al, The Status of Diamond Detectors and a Proposal for $R \& D$ for CDF Beyond Run 3, CDF Note 4233 (1997).

[12] CMS tracker TDR, to be released.

[13] G. Tonnelli et al, NIM A377 (1996) 422.

[14] ATLAS inner detector group, NIM A360 (1995) 432. 
[15] G. Bolla, Pixel98 Presentation.

[16] E. Fretwurst et al, NIM A342 (1994) 119-125.

[17] See

http://www-

cdf.fnal.gov/internal/upgrades/svxii/Mechanical/thermal.html.

[18] D. Amidei and R. Brock, editors, Report of the Tev2000 Study Group on Future Electroweak Physics at the Tevatron, FNAL 1995.

[19] G. Gandi, CERN microcable laboratory.

[20] KY Ng, Power Loss to Tevatron Beam Pipe, FNAL-TM-2015 (1998).

[21] The CDF Collab., A Time-of-Flight System for CDF. CDF Note 2573.

[22] S. Geer, et al., A Description of the Time-of-Flight Test System. CDF Note 3402.

[23] J. Heinrich, et al., Time-of-Flight Test System Performance. CDF Note 3662.

[24] F. Ukegawa, et al., Results from a 20-bar time-of-flight test sytem located inside the 1.4 T CDF solenoid. Submitted to NIM.

[25] Y. Kubota et al. NIM A320, 66-113 (1992).

[26] Kaori Maeshima, Specifications for the CDF II Master Clock System. CDF Note 4509.

[27] M. Jones, J. Kroll, C. Chen, Simulation of a Time-of-Flight Detector for Physics Studies at CDF. CDF Note 4696.

[28] C. Caso et al., The European Physical Journal C3, 1 (1998).

[29] O. R. Long, A Proper Time Dependent Measurement of $\Delta m_{d}$ Using Jet Charge and Soft Lepton Flavor Tagging, Ph.D. dissertation, University of Pennsylvania, 1997 (unpublished);

O. Long, J. Kroll, N. Lockyer, M. Paulini, M. Peters, M. Shapiro, An Update of the Vertex Tagged Inclusive Lepton Data Time Dependent $B^{0}$ Mixing Analysis, CDF Note 4315, Version 1.1.

[30] H. U. Bengtsson and T. Sjöstrand, Computer Physics Commun. 43, 43 (1987);

T. Sjöstrand, Computer Physics Commun. 82, 74 (1994).

We use version 5.702 of PYTHIA. 
[31] P. Sphicas and D. Vučinić, On Using Pythia to Model the Underlying Event in $B$ and $W$ Events, CDF Note 4097;

P. Maksimović, P. Sphicas, D. Vučinić Observation of B(2P) in Semileptonic B Decays, CDF Note 3852.

[32] G. Bauer, K. Kelley, P. Maksimović, P. Sphicas, J. Tseng, F. DeJongh, Comparison of SST distributions from $\ell D^{(*)}$ data and Pythia, CDF Note 4555 .

[33] P. Avery, K. Read, and G. Trahern, Cornell Internal Note CSN-22, 1985 (unpublished).

We use version 9.0 of $\mathrm{QQ}$.

[34] M. Paulini and the LEP B Oscillations Working Group, Combined Results on $B^{0}$ Oscillations: Update for the Summer 1997 Conferences, CDF Note 4297 .

[35] I. Yu and J. Lewis, Two Track Trigger for Run II and Efficiencies for B Hadronic Decays, CDF Note 4095.

[36] F. DeJongh, CDF Sensitivity to $B_{s}$ Oscillations in Run II, CDF Note 4362.

[37] M: Jones, Projections for $x_{s}$ Reach in Run-II Using TOF and Layer 00, CDF Note 4742.

[38] The CDF Collaboration, F. Abe et al., Phys. Rev. D. 58, 5382 (1998).

[39] E. Blucher et al., Proceedings of the Workshop on $B$ physics at Hadron Colliders p. 309, Snowmass, Colorado, 1993.

[40] Y. Nir, hep-ph/9709301.

[41] "Request for SLD Run Extension," the SLD Collaboration, August 1998.

[42] "The BTeV Program at Fermilab," the BTeV Collaboration, BTeV-pub$98 / 3$. 